\title{
PROJECTIVE NORMALITY OF MODEL VARIETIES AND RELATED RESULTS
}

\author{
PAOLO BRAVI, JACOPO GANDINI, AND ANDREA MAFFEI
}

\begin{abstract}
We prove that the multiplication of sections of globally generated line bundles on a model wonderful variety $M$ of simply connected type is always surjective. This follows by a general argument which works for every wonderful variety and reduces the study of the surjectivity for every couple of globally generated line bundles to a finite number of cases. As a consequence, the cone defined by a complete linear system over $M$ or over a closed $G$-stable subvariety of $M$ is normal. We apply these results to the study of the normality of the compactifications of model varieties in simple projective spaces and of the closures of the spherical nilpotent orbits. Then we focus on a particular case proving two specific conjectures of Adams, Huang and Vogan on an analogue of the model orbit of the group of type $\mathrm{E}_{8}$.
\end{abstract}

\section{INTRODUCTION}

Let $G$ be a complex linear algebraic group, semisimple and simply connected. A $G$-variety $M$ is called wonderful of rank $n$ if it satisfies the following conditions:

- $M$ is smooth and projective;

- $M$ possesses an open orbit whose complement is a union of $n$ smooth prime divisors (the boundary divisors) with non-empty transversal intersections;

- any orbit closure in $M$ equals the intersection of the prime divisors which contain it.

Examples of wonderful varieties are the flag varieties, which are the wonderful varieties of rank zero, and the complete symmetric varieties introduced by $\mathrm{C}$. De Concini and C. Procesi [20], which we rather call adjoint symmetric wonderful varieties. Wonderful varieties were then considered in full generality by D. Luna, who started a program of classification in terms of combinatorial invariants 35 .

Consider the following.

Question. Let $M$ be a wonderful variety and, for $\mathcal{L}, \mathcal{L}^{\prime} \in \operatorname{Pic}(M)$, consider the multiplication map

$$
m_{\mathcal{L}, \mathcal{L}^{\prime}}: \Gamma(M, \mathcal{L}) \otimes \Gamma\left(M, \mathcal{L}^{\prime}\right) \longrightarrow \Gamma\left(M, \mathcal{L} \otimes \mathcal{L}^{\prime}\right) .
$$

Is $m_{\mathcal{L}, \mathcal{L}^{\prime}}$ surjective for all globally generated $\mathcal{L}, \mathcal{L}^{\prime}$ ?

In the case of a flag variety, the answer to the previous question is affirmative, indeed by the Borel-Weil theorem $\Gamma(M, \mathcal{L})$ is a simple $G$-module for all $\mathcal{L} \in \operatorname{Pic}(M)$. In the case of the wonderful compactification of an adjoint group (regarded as a symmetric $G \times G$-variety) a still affirmative answer was obtained by S.S. Kannan [27. with a very explicit analysis. In the case of any adjoint symmetric wonderful

Received by the editors February 19, 2015 and, in revised form, December 29, 2015.

2010 Mathematics Subject Classification. Primary 14M27; Secondary 20G05. 
variety the same was obtained by $R$. Chirivì and the third named author in [15] with an inductive argument. In general, when all the above-mentioned multiplication maps are surjective, it follows that the image of $M$ in the dual projective space of the complete linear system associated to any globally generated line bundle is projectively normal.

Another remarkable class of wonderful varieties is that of the model wonderful varieties, introduced by Luna [36. Given a central subgroup $\Gamma \subset G$, a model homogeneous space for the algebraic group $G_{\Gamma}:=G / \Gamma$ is a quasi-affine homogeneous space $G / H$ such that $\Gamma \subset H$ and the coordinate ring $\mathbb{C}[G / H]$ is a model of the representations of $G_{\Gamma}$ in the sense of I.M. Gel'fand (see [4], 24], 25]), that is, $\mathbb{C}[G / H]$ is isomorphic as a $G$-module to the direct sum of all the irreducible representations of $G_{\Gamma}$. Main examples of model homogeneous spaces arise as nilpotent orbits for the adjoint action of $G$; see also [16] where a standard monomial theory for some classical model homogeneous spaces was developed. The model homogeneous spaces for $G_{\Gamma}$ were classified in [36], where Luna constructed a variety $M_{G_{\Gamma}}^{\text {mod }}$, which is wonderful for the action of $G$, whose orbits parametrize the model homogeneous spaces for $G_{\Gamma}$. Varieties of the shape $M_{G_{\Gamma}}^{\text {mod }}$ for some central subgroup $\Gamma \subset G$ are called model wonderful varieties. Given a model wonderful variety $M_{G_{\Gamma}}^{\text {mod }}$, we say that it is of simply connected type if $M_{G_{\Gamma}}^{\text {mod }}$ and $M_{G}^{\text {mod }}$ are $G$-equivariantly isomorphic. For $G$ almost simple (i.e., a non-commutative group having no proper closed connected normal subgroups), it follows by Luna's description that $M_{G_{\Gamma}}^{\text {mod }}$ is not of simply connected type if and only if $G_{\Gamma}=\mathrm{SO}(2 r+1)$ (in which case $G=\operatorname{Spin}(2 r+1)$ and $\Gamma \simeq \mathbb{Z} / 2 \mathbb{Z}$ is the center of $G$ ).

The first result of the paper is the following.

Theorem 1 (see Theorem 4.1). Let $M$ be a model wonderful variety of simply connected type. The multiplication of global sections

$$
m_{\mathcal{L}, \mathcal{L}^{\prime}}: \Gamma(M, \mathcal{L}) \otimes \Gamma\left(M, \mathcal{L}^{\prime}\right) \longrightarrow \Gamma\left(M, \mathcal{L} \otimes \mathcal{L}^{\prime}\right)
$$

is surjective for all globally generated line bundles $\mathcal{L}, \mathcal{L}^{\prime}$ on $M$.

This is false if $M$ is a model wonderful variety not of simply connected type. Indeed, in case $M=M_{\mathrm{SO}(2 r+1)}^{\bmod }$ the multiplication of global sections $m_{\mathcal{L}, \mathcal{L}^{\prime}}$ is surjective for all globally generated line bundles $\mathcal{L}, \mathcal{L}^{\prime}$ on $M$ if and only if $r<4$ (see Section 9.1). This is essentially a consequence of the fact that the tensor product of an almost simple group of type $\mathrm{B}_{r}$ does not satisfy the saturation property in the sense of A. Klyachko (see [31] and 33]).

Theorem 11 follows from a general argument which works for every wonderful variety $M$ and reduces the surjectivity of the maps $m_{\mathcal{L}, \mathcal{L}^{\prime}}$ for all globally generated line bundles $\mathcal{L}, \mathcal{L}^{\prime}$ on $M$ to the surjectivity for a finite number of couples. Indeed, we also prove similar theorems for other classes of wonderful varieties arising in some specific applications.

We now explain in more detail our reduction in the study of the surjectivity of the multiplication maps on a wonderful variety, and how we apply Theorem 1 and its analogues in different directions.

A general reduction. Fix a Borel subgroup $B \subset G$ and a maximal torus $T \subset B$. Denote by $\Sigma$ the set of $G$-stable prime divisors of $M$ and by $\Delta$ the set of $B$-stable prime divisors of $M$ which are not $G$-stable: since $M$ possesses an open $B$-orbit, $\Delta$ is a finite set. Then the Picard group $\operatorname{Pic}(M)$ is freely generated by the line 
bundles $\mathcal{L}_{D}$ with $D \in \Delta$ and the free group $\mathbb{Z} \Sigma$ embeds in $\operatorname{Pic}(M)$, so that we may regard $\mathbb{Z} \Sigma$ as a sublattice of $\mathbb{Z} \Delta$. Moreover, via the isomorphism $\operatorname{Pic}(M) \simeq \mathbb{Z} \Delta$, the semigroup of globally generated line bundles is identified with the semigroup $\mathbb{N} \Delta$. We denote by $\mathcal{L}_{E}$ the globally generated line bundle associated to an element $E \in \mathbb{N} \Delta$. Define the following partial order relation on $\mathbb{N} \Delta$ :

$$
E \leqslant_{\Sigma} F \quad \text { if } \quad E-F \in \mathbb{N} \Sigma \text {. }
$$

This partial order is tightly related to the isotypic decomposition of the spaces of global sections of the globally generated line bundles on $M$, which we may always assume linearized (see Proposition 1.1).

In case $M$ is the wonderful compactification of the adjoint group $G_{\text {ad }}=G / Z(G)$ regarded as a $G \times G$-variety, then $\Sigma$ is naturally identified with the basis of the root system of $G$, while $\Delta$ is naturally identified with the set of the fundamental weights of $G$. More generally, this is true whenever $M$ is the wonderful compactification of a non-Hermitian symmetric space, in which case there always exists a root system $\Phi_{\Sigma}$ (the reduced root system) such that $\Sigma$ is a basis of $\Phi_{\Sigma}$ and $\Delta$ is the corresponding set of fundamental weights. This is no longer true in the case of a general wonderful variety: while there always exists a root system $\Phi_{\Sigma} \subset \mathbb{Z} \Delta$ with $\Sigma$ as set of simple roots, the fundamental weights of $\Phi_{\Sigma}$ associated to $\Sigma$ may differ from $\Delta$. Therefore we may think the couple $(\Sigma, \Delta)$ as a generalization of a root datum.

Suppose that $E, F \in \mathbb{N} \Delta$ are such that $E<_{\Sigma} F$ and there is no $D$ with $E<_{\Sigma}$ $D<_{\Sigma} F$ : then we say that $F-E \in \mathbb{N} \Sigma$ is a covering difference. The set of the covering differences is finite and in the case of an usual root system it was studied by J.R. Stembridge in 41 .

Given $E=\sum_{D \in \Delta} n_{D} D \in \mathbb{Z} \Delta$, define the positive part $E^{+}=\sum_{n_{D}>0} n_{D} D$ and the height $\operatorname{ht}(E)=\sum_{D \in \Delta} n_{D}$. We prove the following.

Lemma 2 (see Lemma 2.3). Let $M$ be a wonderful variety and let $n$ be such that $\mathrm{ht}\left(\gamma^{+}\right) \leqslant n$ for every covering difference $\gamma$. If the multiplication map

$$
m_{\mathcal{L}_{E}, \mathcal{L}_{F}}: \Gamma\left(M, \mathcal{L}_{E}\right) \otimes \Gamma\left(M, \mathcal{L}_{F}\right) \longrightarrow \Gamma\left(M, \mathcal{L}_{E+F}\right)
$$

is surjective for all $E, F \in \mathbb{N} \Delta$ with $h t(E+F) \leqslant n$, then it is surjective for all $E, F \in \mathbb{N} \Delta$.

We use Lemma 2 to prove Theorem 1, We first study the covering relation in the case of a model wonderful variety proving that $h t\left(\gamma^{+}\right) \leqslant 2$ for all covering differences $\gamma$, then we study the multiplication maps $m_{\mathcal{L}_{E}, \mathcal{L}_{F}}$ in the fundamental cases $E, F \in \Delta$. To check the inclusions arising in the exceptional group cases we use the computer.

The fact that $h t\left(\gamma^{+}\right) \leqslant 2$ for all covering differences $\gamma$ is an easy exercise in case the couple $(\Sigma, \Delta)$ corresponds to a root system, and as far as we know it could be a general fact which holds for all wonderful varieties.

Proceeding inductively on the partial order $\leqslant_{\Sigma}$, it is easy to reduce the surjectivity of the multiplication map $m_{\mathcal{L}_{E}, \mathcal{L}_{F}}$ for every $E, F \in \mathbb{N} \Delta$ to the fact that some special submodules of $\Gamma\left(M, \mathcal{L}_{E+F}\right)$ occur in the image of $m_{\mathcal{L}_{E}, \mathcal{L}_{F}}$. This leads to the definition of low triple (see Definition 2.2), which was already introduced in [15] to treat the case of an adjoint symmetric wonderful variety. To prove Lemma 2 we show that it is possible to treat inductively (w.r.t. the height) the low triples of $M$. 
Spherical orbits in simple projective spaces. Our first application of the surjectivity of the multiplication maps regards the study of the normality of the closure of a spherical orbit in a simple projective space, i.e., the projective space of a simple $G$-module. Recall that a $G$-variety $X$ is called spherical if it is normal and possesses an open $B$-orbit, wonderful varieties form a very relevant class of spherical varieties and play a prominent role in their classification.

Let $V$ be a simple $G$-module, let $G \cdot[v] \subset \mathbb{P}(V)$ be a spherical orbit and consider its closure $X=\overline{G \cdot[v]}$, which is not necessarily normal. Let $H$ be the stabilizer of $[v]$, then the spherical homogeneous space $G / H$ can always be embedded as the open orbit of a wonderful variety $M$, called the wonderful compactification of $G / H$, which dominates any other projective compactification of $G / H$ with a unique closed orbit.

If the multiplication of global sections of globally generated line bundles on $M$ is surjective, the normality of every orbit closure $X \subset \mathbb{P}(V)$ dominated by $M$ can be reduced to the normality of some "fundamental" orbit closures. On the other hand, sometimes such fundamental orbit closures are easily shown to be normal. In particular, we have the following.

Theorem 3 (see Corollary 6.9). Let $V$ be a simple $G$-module, let $X \subset \mathbb{P}(V)$ be the closure of a spherical orbit and consider a wonderful variety $M$ which dominates $X$. If $M$ is symmetric with reduced root system of type $\mathrm{A}$ or model for a connected semisimple group of type $\mathrm{AD}$, then $X$ is normal.

Spherical nilpotent orbits. Our second application regards the study of the normality of the closure of a spherical nilpotent orbit $\mathcal{O} \subset \mathfrak{g}$, where $\mathfrak{g}$ denotes the Lie algebra of $G$. In particular, if we consider the projectivization of $\mathcal{O}$, we get again a spherical orbit $\mathcal{U} \subset \mathbb{P}(\mathfrak{g})$, and the closure $\overline{\mathcal{O}}$ coincides with the affine cone over $\overline{\mathcal{U}}$.

Following 13 and a suggestion of D. Luna, to study the normality of $\overline{\mathcal{O}}$ we may consider a wonderful variety $M_{\mathcal{O}}$, namely the wonderful compactification of $\mathcal{U}$. It turns out that the wonderful variety $M_{\mathcal{O}}$ is either symmetric or model, or closely related to one of these. If the surjectivity of the multiplication maps of $M_{\mathcal{O}}$ is known, the description of the $G$-module structure of the coordinate ring of $\overline{\mathcal{O}}$ is an easy task.

Therefore, we prove the following (with computer-aided computations in the exceptional group cases).

Theorem 4 (see Theorem 7.1). Let $\mathcal{O} \subset \mathfrak{g}$ be a spherical nilpotent orbit and let $M_{\mathcal{O}}$ be the associated wonderful variety. Then the multiplication map

$$
m_{\mathcal{L}, \mathcal{L}^{\prime}}: \Gamma\left(M_{\mathcal{O}}, \mathcal{L}\right) \otimes \Gamma\left(M_{\mathcal{O}}, \mathcal{L}^{\prime}\right) \longrightarrow \Gamma\left(M_{\mathcal{O}}, \mathcal{L} \otimes \mathcal{L}^{\prime}\right)
$$

is surjective for all globally generated line bundles $\mathcal{L}, \mathcal{L}^{\prime}$ on $M_{\mathcal{O}}$.

As a corollary (see Corollary 7.2 ) we obtain the classification of the spherical nilpotent adjoint orbits $\mathcal{O}$ whose closure is normal (these results are already known; see for instance [18, Theorem 5.1] or [38, Table 2]). As a particular case, among these orbits we also find the model orbit of $\mathrm{E}_{8}$ studied by J. Adams, J-S. Huang and D.A. Vogan Jr. in [1].

The real model orbit of type $E_{8}$. Following [1, we also consider an analogue of the model nilpotent adjoint orbit $\mathcal{O}$ of $\mathrm{E}_{8}$. More explicitly, we consider a $K$ orbit, where $K$ (an algebraic group) is the complexification of a maximal compact 
subgroup $K_{\mathbb{R}}$ of the split real form of $\mathrm{E}_{8}$. Then $K$ is the fixed point subgroup of an involution of $\mathrm{E}_{8}$, this involution passes to the Lie algebra and $K$ acts on the eigenspace $\mathfrak{p}$ of eigenvalue -1 . The analogue of the model orbit that we consider is the intersection $\mathcal{O}_{\mathfrak{p}}=\mathcal{O} \cap \mathfrak{p}$, which is a $K$-orbit.

For the closure of $\mathcal{O}_{\mathfrak{p}}$ we prove its normality and we describe its coordinate ring (Theorem 8.6). Furthermore, we describe the space of $K_{\mathbb{R}}$-finite vectors of the unitary representation of the split real form of $\mathrm{E}_{8}$ that should be associated to this $K$-orbit via the so-called orbit method (Theorem 8.10). Both descriptions were already present in [1] as consequences of some conjectures, which as far as we know are still open.

In order to prove these theorems, we are led to consider another class of wonderful varieties, which we call comodel wonderful varieties since they are somewhat dual to the model wonderful varieties (see Theorem 5.1 for a precise definition). For the comodel wonderful varieties we also show that the multiplication of global sections of globally generated line bundles is surjective (see Theorem 5.2).

It turns out indeed that $\mathcal{O}_{\mathfrak{p}}$ is the cone over a homogeneous space whose wonderful compactification is closely related to a comodel wonderful variety.

Multiplication of functions on a spherical homogeneous space. Suppose that $G / H$ is a spherical homogeneous space, in the last section of the paper we also consider the problem of multiplying $G$-modules of functions in the coordinate ring $\mathbb{C}[G / H]$. Indeed, $\mathbb{C}[G / H]$ is a multiplicity-free $G$-module (that is, every isotypical component is irreducible), and given two irreducible components $V_{\lambda}$ and $V_{\lambda^{\prime}}$ of highest weight $\lambda$ and $\lambda^{\prime}$ it is well defined their product $V_{\lambda} V_{\lambda^{\prime}} \subset \mathbb{C}[G / H]$, that is, the $G$-module generated by the products $f f^{\prime}$ with $f \in V_{\lambda}$ and $f^{\prime} \in V_{\lambda^{\prime}}$.

More generally, given $\xi \in \mathcal{X}(H)$, one may consider the corresponding $G$-module of the $H$-semi-invariant function

$$
\mathbb{C}[G]_{\xi}^{(H)}=\{f \in \mathbb{C}[G]: f(g h)=\xi(h) f(g) \quad \forall h \in H\},
$$

and given $\xi, \xi^{\prime} \in \mathcal{X}(H)$ there is a natural multiplication map

$$
\mathbb{C}[G]_{\xi}^{(H)} \otimes \mathbb{C}[G]_{\xi^{\prime}}^{(H)} \longrightarrow \mathbb{C}[G]_{\xi+\xi^{\prime}}^{(H)}
$$

As in the case $\xi=0$, the $G$-module $\mathbb{C}[G]_{\xi}^{(H)}$ is multiplicity free, and we denote by $V_{\lambda, \xi}$ its irreducible component of highest weight $\lambda$. Therefore, given $\xi, \xi^{\prime} \in \mathcal{X}(H)$, it is well defined as the product $V_{\lambda, \xi} V_{\lambda^{\prime}, \xi^{\prime}} \subset \mathbb{C}[G]_{\xi+\xi^{\prime}}^{(H)}$.

After discussing some non-degeneracy property of such multiplication and giving some examples, we describe the semigroup generated by the set of differences

$$
\left\{\lambda+\lambda^{\prime}-\mu: V_{\mu, \xi+\xi^{\prime}} \subset V_{\lambda, \xi} V_{\lambda^{\prime}, \xi^{\prime}} \quad \exists \xi, \xi^{\prime} \in \mathcal{X}(H)\right\}
$$

in terms of the spherical roots of a suitable wonderful variety as follows. There is a canonical spherical subgroup of $G$ containing $H$, called the spherical closure of $H$ and denoted $\bar{H}$, such that $G / \bar{H}$ admits a wonderful compactification $M$. Moreover, $\mathcal{X}(H)=\mathcal{X}(\bar{H})$, and for all $\xi \in \mathcal{X}(H)$ it holds that $\mathbb{C}[G]_{\xi}^{(H)}=\mathbb{C}[G]_{\xi}^{(\bar{H})}$. This allows us to reformulate the multiplication of $H$-semi-invariant functions in terms of sections of line bundles on $M$, and we prove the following fact. 
Proposition 5 (see Proposition 9.2). Let $\mathscr{M}$ be the semigroup generated by the set of differences

$$
\left\{\lambda+\lambda^{\prime}-\mu: V_{\mu, \xi+\xi^{\prime}} \subset V_{\lambda, \xi} V_{\lambda^{\prime}, \xi^{\prime}} \quad \exists \xi, \xi^{\prime} \in \mathcal{X}(H)\right\},
$$

and denote by $\Sigma$ the set of spherical roots of $M$. Then $\mathscr{M}=\mathbb{N} \Sigma$.

Structure of the paper. The first part of the paper is entirely devoted to the proof of Theorem 1 and Lemma 2. whereas in the second part we have collected the various applications.

In Section 1 we fix the notation and recall some results about the wonderful varieties and their line bundles. In Section 2 we define the low triples and prove Lemma 2. In Section 3 and in Section 4 we focus on the case of a model wonderful variety, first classifying the covering differences and then classifying the low fundamental triples and studying the associated inclusions.

In Section 5 we introduce the comodel wonderful varieties and prove the surjectivity of the multiplication for this class of varieties.

In Section 6 we explain in general how the surjectivity of the multiplication map can give information on the normality of the closure of a spherical orbit in the projective space of a simple $G$-module (proving, by the way, Theorem 3 ). In Section 7 we prove Theorem 4 and we use our results to deduce the normality and the non-normality of the spherical nilpotent orbit closures. In Section 8 we concentrate on the real model orbit of type $\mathrm{E}_{8}$.

In Section 9 we give the above-mentioned counterexample to the surjectivity of the multiplication in the case of a model wonderful variety of not simply connected type. This leads us to discuss some general properties of the multiplication map and to prove Proposition 5.

\section{Generalities}

Let $G$ be a simply connected semisimple complex algebraic group. Fix a maximal torus $T$ of $G$ and a Borel subgroup $B \supset T$. For any group $K$ we denote by $\mathcal{X}(K)$ the multiplicative characters of $K$. We denote also by $\mathcal{X}(T)^{+}$the set of dominant characters w.r.t. $B$ and if $\lambda \in \mathcal{X}(T)^{+}$we denote by $V(\lambda)$ an irreducible representation of highest weight $\lambda$ and by $-\lambda^{*}$ the lowest weight of $V(\lambda)$. We denote by $S$ the set of simple roots.

Let $M$ be a wonderful $G$-variety with (unique) closed $G$-orbit $Y$. We assume that the center $Z(G)$ acts trivially on $M$, and we will keep this assumption throughout the paper: all the wonderful varieties we will deal with will be $G_{\text {ad-varieties. By }}$ [34], $M$ is spherical, i.e., it possesses an open $B$-orbit, say $B \cdot x_{0} \subset G \cdot x_{0} \subset M$. We denote by $H$ the stabilizer of $x_{0}$ in $G$.

1.1. Colors and spherical roots. Since $B \cdot x_{0}$ is affine, $G \cdot x_{0} \backslash B \cdot x_{0}$ is a union of finitely many $B$-stable prime divisors and we denote by $\Delta$ the set of their closures in $M$ :

$$
\Delta=\left\{D \subset M: D \text { is a } B \text {-stable prime divisor, } D \cap G \cdot x_{0} \neq \varnothing\right\} .
$$

The elements of $\Delta$ are called the colors of $M$.

Denote by $B^{-}$the opposite Borel subgroup of $B$ and let $y_{0} \in Y$ be the unique $B^{-}$-fixed point of $M$. The normal space of $Y$ in $M$ at $y_{0}, \mathrm{~T}_{y_{0}} M / \mathrm{T}_{y_{0}} Y$, is a multiplicity-free $T$-module. Set

$$
\Sigma=\left\{T \text {-weights of } \mathrm{T}_{y_{0}} M / \mathrm{T}_{y_{0}} Y\right\}:
$$


the elements of $\Sigma$ are called the spherical roots of $M$ and they naturally correspond to local equations of the boundary divisors of $M$, which are $G$-stable. If $\sigma \in \Sigma$, we denote by $M^{\sigma}$ the associated boundary divisor of $M$ such that $\mathrm{T}_{y_{0}} M / \mathrm{T}_{y_{0}} M^{\sigma}$ is the 1-dimensional $T$-module of weight $\sigma$.

The rank one wonderful varieties were classified by D.N. Akhiezer [2], and we denote by $\Sigma(G) \subset \mathcal{X}(T)$ the finite set of all possible weights occurring as the spherical root of a rank one wonderful variety. These are called the spherical roots of $G$. Every spherical root is either a positive root, or the sum of two orthogonal positive roots, we refer to [8, Table 1] for a list of the spherical roots of $G$.

1.2. Picard group and Cartan pairing. Recall that every line bundle on $M$ or on $Y$ has a unique $G$-linearization.

We may identify $\operatorname{Pic}(Y)$ with a sublattice of $\mathcal{X}(T)$ and $\operatorname{Pic}\left(G \cdot x_{0}\right)$ with $\mathcal{X}(H)$ (see [30]): we identify $\mathcal{L} \in \operatorname{Pic}(Y)$ with the character of $T$ acting on the fiber of $\mathcal{L}$ over $y_{0}$, and we identify $\mathcal{L} \in \operatorname{Pic}\left(G \cdot x_{0}\right)$ with the character of $H$ acting on the fiber over $x_{0}$.

Consider now the maps $\omega: \operatorname{Pic}(M) \longrightarrow \mathcal{X}(T)$ and $\xi: \operatorname{Pic}(M) \longrightarrow \mathcal{X}(H)$ defined by the restriction to the closed and to the open orbit. We may regard $\operatorname{Pic}(M)$ as a sublattice of $\mathcal{X}(T) \times \mathcal{X}(H)$ by identifying $\mathcal{L} \in \operatorname{Pic}(M)$ with the couple $(\omega(\mathcal{L}), \xi(\mathcal{L}))$ (see [1]). Moreover, we have the following exact sequence

$$
0 \longrightarrow \mathbb{Z} \Sigma \longrightarrow \operatorname{Pic}(M) \longrightarrow \mathcal{X}(H) \longrightarrow 0 .
$$

Given $E \in \mathbb{Z} \Delta$, denote by $\mathcal{L}_{E}:=\mathcal{O}(E)$ the associated line bundle. As a group, $\operatorname{Pic}(M)$ is freely generated by the line bundles $\mathcal{L}_{D}$ with $D \in \Delta$ (see [10, Proposition 2.2]). For all $E \in \mathbb{Z} \Delta$, the associated line bundle $\mathcal{L}_{E}$ is globally generated (resp. ample) if and only if $E$ is a non-negative (resp. positive) combination of colors. We set $\omega_{E}=\omega\left(\mathcal{L}_{E}\right), \xi_{E}=\xi\left(\mathcal{L}_{E}\right)$.

There exists a natural $\mathbb{Z}$-bilinear pairing (called the Cartan pairing of $M$ )

$$
c: \mathbb{Z} \Delta \times \mathbb{Z} \Sigma \longrightarrow \mathbb{Z}
$$

which maps the couple $(D, \sigma)$ to the coefficient of $\left[M^{\sigma}\right]$ along $[D]$. So, regarding $\mathbb{Z} \Sigma$ as a sublattice of $\mathbb{Z} \Delta$, for $\sigma \in \mathbb{Z} \Sigma$ we have

$$
\sigma=\sum_{D \in \Delta} c(D, \sigma) D .
$$

1.3. Global sections and multiplication. We now recall the description of the space of global sections $\Gamma(M, \mathcal{L})$ of a line bundle $\mathcal{L}$. Notice first that since $M$ is spherical the decomposition of $\Gamma(M, \mathcal{L})$ into simple $G$-modules is multiplicity free for all $\mathcal{L} \in \operatorname{Pic}(M)$. If $E \in \mathbb{N} \Delta$ then $\mathcal{L}_{E}$ is generated by global sections. In particular $\Gamma\left(M, \mathcal{L}_{E}\right)$ must contain a copy of $V\left(\omega_{E}\right)$ (which is the space of sections of $\mathcal{L}_{E}$ on $Y)$, hence $\omega_{E}$ is dominant. We denote by $V_{E}$ the unique simple $G$-submodule of $\Gamma\left(M, \mathcal{L}_{E}\right)$ of highest weight $\omega_{E}$. Notice also that the image of $x_{0} \operatorname{in} \mathbb{P}\left(\Gamma\left(M, \mathcal{L}_{E}\right)^{*}\right)$ is a point fixed by $H$. In particular, since $B H \subset G$ is open, it follows that the space of spherical vectors

$$
V\left(\omega_{E}^{*}\right)_{\xi_{E}}^{(H)}=\left\{v \in V\left(\omega_{E}^{*}\right): h v=\xi_{E}(h) v \quad \forall h \in H\right\}
$$

has dimension one and we denote by $h_{E}$ a generator of this line.

If $\gamma=\sum a_{\sigma} \sigma \in \mathbb{N} \Sigma$, we denote by $s^{\gamma} \in \Gamma\left(M, \mathcal{L}_{M^{\gamma}}\right)$ a section whose divisor is equal to $M^{\gamma}=\sum a_{\sigma} M^{\sigma}$. Notice that this section is $G$-invariant. Recall, as defined in the introduction, that we say that $F \leqslant_{\Sigma} E$ if $E-F \in \mathbb{N} \Sigma$. If $E \in \mathbb{N} \Delta$ and 
$E \leqslant_{\Sigma} F$ the multiplication by $s^{F-E}$ induces a $G$-equivariant map from the sections of $\mathcal{L}_{E}$ to the sections of $\mathcal{L}_{F}$, in particular, we have $s^{F-E} V_{E} \subset \Gamma\left(M, \mathcal{L}_{F}\right)$.

Proposition 1.1 ([10, Proposition 2.4]). Let $F \in \mathbb{Z} \Delta$. Then

$$
\Gamma\left(M, \mathcal{L}_{F}\right)=\bigoplus_{E \in \mathbb{N} \Delta: E \leqslant \Sigma} s^{F-E} V_{E}
$$

If $E, F \in \mathbb{N} \Delta$, consider the multiplication of sections

$$
m_{E, F}: \Gamma\left(M, \mathcal{L}_{E}\right) \otimes \Gamma\left(M, \mathcal{L}_{F}\right) \longrightarrow \Gamma\left(M, \mathcal{L}_{E+F}\right)
$$

and denote by $V_{E} V_{F}$ the image of $V_{E} \otimes V_{F}$. A way to translate the description of this map into a problem on spherical vectors is the following.

Lemma 1.2 ([14, Lemma 19]). Let $D, E, F \in \mathbb{N} \Delta$ be such that $D \leqslant_{\Sigma} E+F$. Then $s^{E+F-D} V_{D} \subset V_{E} V_{F}$ if and only if the projection of $h_{E} \otimes h_{F} \in V\left(\omega_{E}^{*}\right) \otimes V\left(\omega_{F}^{*}\right)$ onto the isotypic component of highest weight $\omega_{D}^{*}$ is non-zero.

1.4. Distinguished sets of colors. Let $M^{\prime}$ be a wonderful $G$-variety together with a surjective equivariant morphism $\phi: M \longrightarrow M^{\prime}$ with connected fibers and denote $\Delta_{\phi} \subset \Delta$ the set of colors which map dominantly onto $M^{\prime}$. Then the semigroup

$$
(\mathbb{N} \Sigma) / \Delta_{\phi}=\left\{\gamma \in \mathbb{N} \Sigma: c(D, \gamma)=0 \quad \forall D \in \Delta_{\phi}\right\}
$$

is free and its basis, which we denote by $\Sigma / \Delta_{\phi}$, coincides with the set of spherical roots of $M^{\prime}$, while the set of colors of $M^{\prime}$ is identified with $\Delta \backslash \Delta_{\phi}$ (see [35, Proposition 3.3.2]).

Conversely, if $\Delta_{0} \subset \Delta$, then there exists a wonderful variety $M^{\prime}$ (unique up to isomorphism) together with a surjective equivariant morphism $\phi: M \longrightarrow M^{\prime}$ with connected fibers if and only if $\Delta_{0}$ is distinguished, that is, there exists an element $D \in \mathbb{N}_{>0} \Delta_{0}$ such that $c(D, \sigma) \geqslant 0$ for every $\sigma \in \Sigma$ (see [35. Proposition 3.3.2] and [5. Theorem 3.1]).

If $\Delta_{0} \subset \Delta$ is distinguished, then we say that the associated wonderful variety $M^{\prime}$ is the quotient of $M$ by $\Delta_{0}$, denoted by $M / \Delta_{0}$.

Recall the following general fact.

Proposition 1.3. Let $X, Y$ be normal varieties and suppose that $\phi: X \longrightarrow Y$ is a surjective proper morphism with connected fibers. If $\mathcal{L} \in \operatorname{Pic}(Y)$, then $\Gamma(Y, \mathcal{L})=$ $\Gamma\left(X, \phi^{*} \mathcal{L}\right)$.

If $E=\sum_{D \in \Delta} a_{D} D \in \mathbb{Z} \Delta$ we define $\operatorname{supp}(E)$, the support of $E$, as the set of colors $D$ such that $a_{D} \neq 0$.

Corollary 1.4. Let $E \in \mathbb{N} \Delta$ and suppose that $\Delta_{0}$ is a distinguished subset of $\Delta$ such that $\Delta_{0} \cap \operatorname{supp}(E)=\varnothing$. Then $\Gamma\left(M, \mathcal{L}_{E}\right)=\Gamma\left(M / \Delta_{0}, \mathcal{L}_{E}\right)$.

1.5. Parabolic induction: spherical roots and colors. We describe now a standard way to construct a wonderful variety for the group $G$ from a wonderful variety for a Levi subgroup of a parabolic subgroup of $G$.

Let $L$ be a proper Levi subgroup of $G$ which contains $T$. Let $Q$ be the parabolic subgroup associated to $L$ and containing $B$, and let $Q^{-}$be the opposite parabolic subgroup. Denote by $R_{Q}$ and $R_{Q}^{-}$the solvable radicals and by $U_{Q}$ and $U_{Q}^{-}$the unipotent radicals of $Q$ and $Q^{-}$, respectively. Finally let $L^{\text {ss }}$ be the semisimple part of $L$ and denote by $L_{\mathrm{ad}}=L^{\mathrm{ss}} / Z\left(L^{\mathrm{ss}}\right)=L / Z(L)$ the adjoint quotient of $L$. 
Let $N$ be a wonderful variety for the group $L_{\mathrm{ad}}$. Although wonderful varieties have been defined for semisimple groups, in this case it is convenient to look at $N$ as an $L$-variety and to consider $L$-linearized line bundles on $N$. The definitions given for a wonderful variety can be extended to this situation.

Extend the action of $L$ on $N$ to $Q^{-}$, with $U_{Q}^{-}$acting trivially, and define the parabolic induction of $N$ as

$$
M=G \times{ }_{Q^{-}} N .
$$

We have a projection $\pi: M \longrightarrow G / Q^{-}, N$ is the fiber over $Q^{-}$and, moreover, it is the set of points of $M$ fixed by $U_{Q}^{-}$. One can easily show the following properties.

(1) $M$ is a wonderful $G$-variety.

(2) The intersection of a subset of $M$ with $N$ induces a bijection from the set of $G$-stable subsets of $M$ to the set of $L$-stable subsets of $N$. For every spherical root $\sigma$ of $M$ we denote by $N^{\sigma}$ the intersection $M^{\sigma} \cap N$.

(3) The restriction of line bundles from $M$ to $N$ induces an isomorphism $\rho$ between the class groups of linearized line bundles $\operatorname{Pic}_{G}(M)$ and $\operatorname{Pic}_{L}(N)$.

(4) We have an injective map $\varepsilon$ from the set $\Delta(N)$ of colors of $N$ to the set $\Delta(M)$ of colors of $M$ given by $\varepsilon(D)=\overline{B \cdot D}$ for all $D \in \Delta(N)$.

(5) The exact sequence $0 \longrightarrow \mathcal{X}\left(L / L^{\mathrm{ss}}\right) \longrightarrow \operatorname{Pic}_{L}(N) \longrightarrow \operatorname{Pic}_{L^{\mathrm{ss}}}(N) \longrightarrow 0$ has a natural splitting given by $\left.\mathcal{L}_{D} \longmapsto \mathcal{L}_{\varepsilon(D)}\right|_{N}$ for every color $D \in \Delta(N)$.

(6) If $D \in \Delta(M) \backslash \varepsilon(\Delta(N))$ then the restriction of $\mathcal{O}(D)$ to $N$ is a trivial line bundle on $N$.

(7) The set of colors $\varepsilon(\Delta(N))$ is distinguished and $M / \varepsilon(\Delta(N)) \simeq G / Q^{-}$.

(8) Since $L^{\mathrm{ss}}$ is simply connected we have $\operatorname{Pic}(N) \simeq \operatorname{Pic}_{L^{\mathrm{ss}}}(N)$.

Denote

$$
\Sigma=\left\{T \text {-weights of } \mathrm{T}_{y_{0}} N / \mathrm{T}_{y_{0}}\left(L \cdot y_{0}\right)\right\},
$$

where we recall that $y_{0}$ is the point of $M$ fixed by $B^{-}$, hence $L \cdot y_{0}$ is the unique closed orbit of $N$. The set $\Sigma$ is in correspondence with the set of $L$-stable divisors and, in particular, $\sigma$ is the $T$-weight of $\mathrm{T}_{y_{0}} N / \mathrm{T}_{y_{0}} N^{\sigma}$. Moreover, we have that $\Sigma$ equals the set of spherical roots of $M$ and every element of $\Sigma$ is a sum of simple roots of $L$.

By the above properties (3) and (4) for every color $D$ in $\Delta(N)$ we have a canonical choice of a linearization of the associated line bundle and we have a natural decomposition

$$
\operatorname{Pic}_{L}(N) \simeq \mathcal{X}\left(L / L^{\mathrm{ss}}\right) \oplus \bigoplus_{D \in \Delta(N)} \mathbb{Z}[D] .
$$

For all $\sigma \in \Sigma,\left[N^{\sigma}\right]=\sum_{D \in \Delta(M)} c(D, \sigma) \rho([D])$. If $\mathcal{L}, \mathcal{L}^{\prime} \in \operatorname{Pic}_{L}(N)$ we define $\mathcal{L} \geqslant_{\Sigma} \mathcal{L}^{\prime}$ if, using the additive notation, $\mathcal{L}-\mathcal{L}^{\prime}=\sum a_{\sigma} \mathcal{O}\left(N^{\sigma}\right)$ with $a_{\sigma} \geqslant 0$ for all $\sigma$, similarly to what we have done in Section 1.3. In this way Proposition 1.1 holds for $N$ without any change.

It is easy to see when a $G$-wonderful variety $M$ can be obtained by parabolic induction. Let $P^{-}$be the stabilizer of $y_{0}$ and let $S^{p}$ the set of simple roots that are in the set of roots of $P^{-}$. Assume that $S^{\prime}=S^{p} \cup \operatorname{supp}_{S} \Sigma \neq S$ and let $L$ be the standard Levi subgroup associated to $S^{\prime}$. Let $Q, Q^{-}, R_{Q}, R_{Q}^{-}, U_{Q}, U_{Q}^{-}, L^{\mathrm{ss}}, L_{\mathrm{ad}}$ be defined as above. Set $\widetilde{\Delta}=\left\{D \in \Delta\right.$ : if $P_{\alpha} \cdot D \neq D$ then $\left.\alpha \in S^{\prime}\right\}$, then $\widetilde{\Delta}$ is a distinguished set and $M / \widetilde{\Delta}=G / Q^{-}$. Moreover, $N=M^{U_{Q}^{-}}$is a wonderful variety for $L_{\text {ad }}$ and $M$ is obtained by parabolic induction from $N$ as above. 
1.6. Parabolic induction: global sections. Now let $M$ be obtained by parabolic induction from $N$ as above. Let $\mathcal{M}$ be a line bundle on $M$ and denote by $\mathcal{N}$ its restriction to $N$. We want to compare the sections of $\mathcal{M}$ and $\mathcal{N}$. The restriction of sections induces a map $r_{\mathcal{M}}: \Gamma(M, \mathcal{M}) \longrightarrow \Gamma(N, \mathcal{N})$. Notice that $U \cdot N$ is a dense subset of $M$, hence the restriction of $r_{\mathcal{M}}$ to $\Gamma(M, \mathcal{M})^{U}$ is injective. We first describe the kernel of $r_{\mathcal{M}}$.

Let $\mathcal{M}=\mathcal{L}_{F}$, then $\Gamma(M, \mathcal{M})=\bigoplus s^{F-E} V_{E}$ where the sum is over all $E \in \mathbb{N} \Delta$ such that $E \leqslant_{\Sigma} F$. For each $E \in \mathbb{N} \Delta$ set $W_{E}=V_{E}^{U_{Q}}$. This is an irreducible $L$ submodule with the same highest weight as $V_{E}$. Let $I_{E}$ be the $L$-stable complement of $W_{E}$ in $V_{E}$.

Lemma 1.5. We have

$$
\operatorname{ker} r_{\mathcal{M}}=\bigoplus_{E \in \mathbb{N} \Delta: E \leqslant \Sigma F} s^{F-E} I_{E}
$$

Proof. By the above discussion it is enough to prove that $r_{\mathcal{L}_{E}}\left(I_{E}\right)=0$. Let $v$ be a highest weight vector (for the action of $L$ ) in $I_{E}$ of weight $\lambda$. Then $\omega_{E}-\lambda=$ $\sum_{\alpha \in S} a_{\alpha} \alpha$ with $a_{\alpha} \in \mathbb{N}$. If $S^{\prime}$ is the set of simple roots for $L$ notice that, since $v \in I_{E}$, there exists $\alpha \in S \backslash S^{\prime}$ such that $a_{\alpha} \neq 0$. On the other hand, by the generalization of Proposition 1.1 for $N$ discussed in the previous section, the weights of the highest weight vectors in $\Gamma\left(N, \mathcal{L}_{E}\right)$ are of the form $\omega_{E}-\beta$ with $\beta \in \mathbb{N} S^{\prime}$. In particular, we must have $r_{\mathcal{L}_{E}}(v)=0$.

Since the restriction commutes with the multiplication we recover the following.

Proposition 1.6 ([15, Proposition 2.9]). For all $E, F \in \mathbb{N} \Delta$ and $\gamma \in \mathbb{N} \Sigma$ such that $E+F-\gamma \in \mathbb{N} \Delta, s^{\gamma} V_{E+F-\gamma} \subset V_{E} V_{F}$ if and only if $s^{\gamma} W_{E+F-\gamma} \subset W_{E} W_{F}$. ing.

In particular, using the property (6) of the previous section, we have the follow-

Corollary 1.7. For all $D \in \mathbb{N}(\Delta \backslash \widetilde{\Delta})$ and $E \in \mathbb{N} \Delta, V_{D} V_{E}=V_{D+E}$.

\section{Projective Normality and the COVERING Relation}

2.1. The covering relation. Let $\left\{\omega_{\alpha}: \alpha \in S\right\}$ be the set of fundamental weights w.r.t. the simple roots $S$.

For all $\lambda=\sum k_{\alpha} \omega_{\alpha} \in \mathcal{X}(T)$, denote by $\operatorname{supp}(\lambda)$ the set of $\alpha \in S$ such that $k_{\alpha} \neq 0$ and define its positive part $\lambda^{+}$, respectively, its negative part $\lambda^{-}$, as the dominant weights

$$
\lambda^{+}=\sum_{k_{\alpha}>0} k_{\alpha} \omega_{\alpha} \quad \text { and } \quad \lambda^{-}=\lambda^{+}-\lambda .
$$

If $\lambda \in \mathcal{X}(T)^{+}$, define also the height of $\lambda$ as the number $\operatorname{ht}(\lambda)=\sum_{\alpha \in S} k_{\alpha}$.

Suppose that $\lambda$ and $\mu$ are dominant weights with $\lambda<\mu$ (w.r.t. the usual dominance order) and suppose that there is no dominant weight $\nu$ such that $\lambda<\nu<\mu$ : then one says that $\mu$ covers $\lambda$ and we call $\mu-\lambda$ a covering difference in $\mathcal{X}(T)^{+}$. Notice that an element $\gamma \in \mathbb{N} S$ is a covering difference in $\mathcal{X}(T)^{+}$if and only if $\gamma^{+}$covers $\gamma^{-}$. Although the following proposition is an immediate consequence of [41, Theorem 2.6], here we give an easy independent proof.

Proposition 2.1. If $\gamma \in \mathbb{N} S$ is a covering difference in $\mathcal{X}(T)^{+}$, then $\operatorname{ht}\left(\gamma^{+}\right) \leqslant 2$. 
Proof. Let $\gamma \in \mathbb{N} S$ be a covering difference and suppose that $\alpha \in S$ is such that $\left\langle\gamma^{+}, \alpha^{\vee}\right\rangle \geqslant 2$ : then $\gamma^{+}-\alpha \in \mathcal{X}(T)^{+}$, hence we must have $\gamma=\alpha$ and $\operatorname{ht}\left(\gamma^{+}\right)=$ 2. Hence we may assume that $\left\langle\gamma^{+}, \alpha^{\vee}\right\rangle \leqslant 1$ for every $\alpha \in S$. Suppose that ht $\left(\gamma^{+}\right) \geqslant 3$, (up to reindexing the simple roots) we can take $\alpha_{i}, \alpha_{j} \in \operatorname{supp}_{S}\left(\gamma^{+}\right)$ with $i<j$ such that $\left\{\alpha_{i}, \ldots, \alpha_{j}\right\}$ generates an irreducible subsystem of type A; then $\gamma^{+}-\left(\alpha_{i}+\cdots+\alpha_{j}\right) \in \mathcal{X}(T)^{+}$and it follows $\gamma=\alpha_{i}+\cdots+\alpha_{j}$ and ht $\left(\gamma^{+}\right)=2$ (a contradiction).

We now consider the covering relation in the more general context of wonderful varieties. Let $M$ be a wonderful variety with set of spherical roots $\Sigma$ and with set of colors $\Delta$. For all $E=\sum_{D \in \Delta} k_{D} D \in \mathbb{Z} \Delta$, define its positive part $E^{+}$and its negative part $E^{-}$as

$$
E^{+}=\sum_{k_{D}>0} k_{D} D \quad \text { and } \quad E^{-}=E^{+}-E .
$$

If $E \in \mathbb{N} \Delta$, define the height of $E$ as the number ht $(E)=\sum_{D \in \Delta} k_{D}$. On the other hand, for all $\gamma=\sum_{\sigma \in \Sigma} a_{\sigma} \sigma \in \mathbb{N} \Sigma$ define its $\Sigma$-height as ht ${ }_{\Sigma}(\gamma)=\sum_{\sigma \in \Sigma} a_{\sigma}$.

Let $E$ and $F$ be in $\mathbb{N} \Delta$ with $E<_{\Sigma} F$ and suppose that there is no $D \in \mathbb{N} \Delta$ such that $E<_{\Sigma} D<_{\Sigma} F$; then we say that $F$ covers $E$ and we call $F-E$ a covering difference in $\mathbb{N} \Delta$. Again, $\gamma \in \mathbb{N} \Sigma$ is a covering difference in $\mathbb{N} \Delta$ if and only $\gamma^{+}$ covers $\gamma^{-}$.

Remark 1. Notice that there are only finitely many covering differences. Indeed, consider the lattice $X=\{(D, E) \in \mathbb{Z} \Delta \times \mathbb{Z} \Delta: E-D \in \mathbb{Z} \Sigma\}$ and the finitely generated monoid $X^{+}=\{(D, E) \in \mathbb{N} \Delta \times \mathbb{N} \Delta: D-E \in \mathbb{N} \Sigma\}$. If $\gamma$ is a covering difference then $\left(\gamma^{+}, \gamma^{-}\right)$is an indecomposable element of $X^{+}$.

Finally, the number of indecomposable elements in $X^{+}$is finite and can be controlled as follows: let $\ell_{1}, \ldots, \ell_{t}$ be the half-lines generating the cone $X_{\mathbb{Q}}^{+}$, let $v_{i}=\left(D_{i}, E_{i}\right)$ be a generator of $\ell_{i} \cap X^{+}$and let $n_{i}=\operatorname{ht}\left(D_{i}\right)+\operatorname{ht}\left(E_{i}\right)$. Then if $(D, E)$ is indecomposable, ht $(D)+\operatorname{ht}(E) \leqslant \sum_{i} n_{i}$.

As already said in the introduction, in the case of the wonderful compactification of a non-Hermitian symmetric space, there exists a root system $\Phi_{\Sigma}$ (the restricted root system) which is generated by the spherical roots and such that $\Delta$ is naturally identified with the set of fundamental weights of $\Phi_{\Sigma}$ and the pairing between $\Sigma$ and $\Delta$ is the Cartan pairing of $\Phi_{\Sigma}$. Although $\Sigma$ is always the basis of a root system $\Phi_{\Sigma}$, in the general case it is not possible to identify $\Delta$ with the fundamental weights of $\Phi_{\Sigma}$; in particular, it may happen that $\mathbb{Z} \Sigma \subset \mathbb{Z} \Delta$ is not a sublattice of finite index and that the semigroup of dominant weights of $\Phi_{\Sigma}$ contained in $\mathbb{Z} \Sigma$ is not even contained in $\mathbb{N} \Delta$. However, one can consider the property of Proposition 2.1 without modifications in this context:

(2-ht) If $\gamma \in \mathbb{N} \Sigma$ is a covering difference in $\mathbb{N} \Delta$, then $h t\left(\gamma^{+}\right) \leqslant 2$.

Notice that by the above discussion the property (2-ht) holds if $M$ is the wonderful compactification of a non-Hermitian symmetric space. In the case of the wonderful compactifications of Hermitian symmetric spaces the same argument works without serious complications. In the subsequent section we show that it is true also in the case of a model wonderful variety (of simply connected type). We have also checked many other examples and, as far as we know, it is possible that it holds for all wonderful varieties. 
In analogy with the case of a root system, we say that a non-zero element $D \in \mathbb{N} \Delta$ is minuscule if it is minimal in $\mathbb{N} \Delta$ w.r.t. the partial order $\leqslant_{\Sigma}$.

2.2. Projective normality. The notion of low triple has been introduced in [15] in the case of a symmetric wonderful variety. Here we use the same terminology with a slightly weaker definition.

Definition 2.2. Let $M$ be a wonderful variety with set of spherical roots $\Sigma$ and with set of colors $\Delta$. Let $D, E, F \in \mathbb{N} \Delta$ be such that $F \leqslant \Sigma D+E$. The triple $(D, E, F)$ is called a low triple if:

for all $D^{\prime} \leqslant_{\Sigma} D$ and $E^{\prime} \leqslant_{\Sigma} E$ such that $F \leqslant_{\Sigma} D^{\prime}+E^{\prime}$, it holds that $D^{\prime}=D$ and $E^{\prime}=E$.

The triple $(D, E, F)$ is called a fundamental triple if $D, E \in \Delta$.

Notice that if $(D, E, F)$ is a low triple, then $s^{D+E-F} V_{F} \subset \Gamma\left(M, \mathcal{L}_{D}\right) \Gamma\left(M, \mathcal{L}_{E}\right)$ if and only if $s^{D+E-F} V_{F} \subset V_{D} V_{E}$.

Lemma 2.3. Let $M$ be a wonderful variety and let $n$ be such that $\operatorname{ht}\left(\gamma^{+}\right) \leqslant n$ for every covering difference $\gamma$. If

$$
s^{D+E-F} V_{F} \subset V_{D} V_{E},
$$

for all low triples $(D, E, F)$ with $\mathrm{ht}(D+E) \leqslant n$, then the multiplication map

$$
m_{D, E}: \Gamma\left(M, \mathcal{L}_{D}\right) \otimes \Gamma\left(M, \mathcal{L}_{E}\right) \longrightarrow \Gamma\left(M, \mathcal{L}_{D+E}\right)
$$

is surjective for all $D, E \in \mathbb{N} \Delta$.

Proof. For any $D \in \mathbb{Z} \Delta$ let $\Gamma_{D}=\Gamma\left(M, \mathcal{L}_{D}\right)$. For any triple $(D, E, F)$ with $F \leqslant \Sigma$ $D+E$ we want to show that $s^{D+E-F} V_{F} \subset \Gamma_{D} \Gamma_{E}$. We proceed by induction first on $\operatorname{ht}_{\Sigma}(D+E-F)$ and then on $\operatorname{ht}(D+E)$. If ht $(D+E-F)=0$ the claim is trivial.

If $(D, E, F)$ is not a low triple then there exist $D^{\prime} \leqslant_{\Sigma} D$ and $E^{\prime} \leqslant_{\Sigma} E$ such that $F \leqslant_{\Sigma} D^{\prime}+E^{\prime}$ and $\mathrm{ht}_{\Sigma}\left(D^{\prime}+E^{\prime}-F\right)<\mathrm{ht}_{\Sigma}(D+E-F)$. Hence the claim is true for $\left(D^{\prime}, E^{\prime}, F\right)$, and

$$
s^{D+E-F} V_{F}=s^{D-D^{\prime}+E-E^{\prime}} s^{D^{\prime}+E^{\prime}-F} V_{F} \subset s^{D-D^{\prime}+E-E^{\prime}} \Gamma_{D^{\prime}} \Gamma_{E^{\prime}} \subset \Gamma_{D} \Gamma_{E} .
$$
tion.

If $(D, E, F)$ is a low triple and $\operatorname{ht}(D+E) \leqslant n$ then the claim is true by assump-

Assume now that $\operatorname{ht}(D+E)>n$ and that $(D, E, F)$ is a low triple. Let $F_{1} \in \mathbb{N} \Delta$ be an element covered by $D+E$ such that $F_{1} \geqslant_{\Sigma} F$. Since $\operatorname{ht}(D+E)>\operatorname{ht}\left(\gamma^{+}\right)$ for every covering difference $\gamma \in \mathbb{N} \Sigma$, it follows that $\operatorname{supp}\left(F_{1}\right) \cap \operatorname{supp}(D+E) \neq \varnothing$. Fix $D_{0} \in \operatorname{supp}\left(F_{1}\right) \cap \operatorname{supp}(D+E)$ and set

$$
\left(D_{1}, E_{1}\right)= \begin{cases}\left(D-D_{0}, E\right) & \text { if } D_{0} \in \operatorname{supp}(E), \\ \left(D, E-D_{0}\right) & \text { otherwise. }\end{cases}
$$

Choose $F_{2}$ in $\mathbb{N} \Delta$ minimal w.r.t. $\leqslant \Sigma$ such that $F-D_{0} \leqslant \Sigma F_{2} \leqslant \Sigma F_{1}-D_{0}$. Notice that $\operatorname{ht}_{\Sigma}\left(D_{0}+F_{2}-F\right) \leqslant \operatorname{ht}_{\Sigma}\left(F_{1}-F\right)<\mathrm{ht}_{\Sigma}(D+E-F)$. Moreover, $\left(D_{0}, F_{2}, F\right)$ is a low triple; indeed if there exist $F_{2}^{\prime} \leqslant \Sigma F_{2}$ and $D_{0}^{\prime} \leqslant \Sigma D_{0}$ in $\mathbb{N} \Delta$ such that $F \leqslant \Sigma D_{0}^{\prime}+F_{2}^{\prime}$, then

$$
F \leqslant \leqslant_{\Sigma} D_{0}^{\prime}+F_{2}^{\prime} \leqslant_{\Sigma} D_{0}^{\prime}+D_{1}+E_{1} \leqslant \Sigma D+E .
$$

Since $(D, E, F)$ is a low triple, we get $D_{0}^{\prime}=D_{0}$, while since $F_{2}$ is minimal we get $F_{2}^{\prime}=F_{2}$. Hence, by induction, it follows that $s^{D_{0}+F_{2}-F} V_{F} \subset V_{D_{0}} V_{F_{2}}$. 
Notice that we have ht ${ }_{\Sigma}\left(D_{1}+E_{1}-F_{2}\right) \leqslant \operatorname{ht}_{\Sigma}(D+E-F)$ and that ht $\left(D_{1}+E_{1}\right)=$ ht $(D+E)-1$, hence we can apply the inductive hypothesis to $\left(D_{1}, E_{1}, F_{2}\right)$. So $s^{D+E-F} V_{F}=s^{D_{1}+E_{1}-F_{2}} s^{D_{0}+F_{2}-F} V_{F} \subset s^{D_{1}+E_{1}-F_{2}} V_{F_{2}} V_{D_{0}} \subset \Gamma_{D_{1}} \Gamma_{E_{1}} V_{D_{0}} \subset \Gamma_{D} \Gamma_{E}$.

Together with Remark 1, the lemma implies that to prove the surjectivity of $m_{D, E}$ for all $D, E \in \mathbb{N} \Delta$ it is enough to check a finite number of cases. In particular if the property (2-ht) holds, it is enough to check the above inclusion (2.1) only for the low fundamental triples.

In Section 9.1 we show that there exist wonderful varieties possessing low fundamental triples $(D, E, F)$ such that $s^{D+E-F} V_{F} \not \subset V_{D} V_{E}$; in particular, the multiplication of sections of line bundles on a wonderful variety is not necessarily surjective.

\section{THE COVERING RELATION FOR MODEL WONDERFUL VARIETIES}

As said in the introduction, a model homogeneous space for a reductive group $G$ (not necessarily simply connected) is a quasi affine $G$-homogeneous space whose coordinate ring is a model representation of $G$ : each irreducible representation of $G$ appears exactly with multiplicity one. By [36, for every group $G$ there exists a wonderful variety $M$ such that for every model homogenous space $G / H_{0}$ there exists a point in $M$ whose stabilizer is equal to $N_{G}\left(H_{0}\right)$ and, vice versa, if $H$ is the stabilizer of a point in $M$ and we set $H_{0}$ to be the intersections of the kernels of the multiplicative characters of $H$, then $G / H_{0}$ is a model homogeneous space. The variety $M$ is called the model wonderful variety of $G$.

Notice that $H_{0}$ has no characters, hence the set of spherical vectors (i.e., the eigenvectors of $H$ ) in a simple $G$-module $V$, which is a subspace of dimension at most one, can be also characterized as the set of vectors in $V$ fixed by $H_{0}$.

The description of the model wonderful varieties of an almost simple group $G$ is given in 36 . For type $\mathrm{B}$ there are two different model wonderful varieties, according to whether $G$ is isomorphic to the special orthogonal group or to the spin group. In all the other cases the model wonderful variety of $G$ is the same as the model wonderful variety of the adjoint group of $G$.

In this section we describe the covering relation for a model wonderful variety $M$ of simply connected type, and we prove that the property (2-ht) holds in this case. For model wonderful varieties of simply connected type the set of colors $\Delta$ is in bijection, via $\omega$, with the set of fundamental weights, and the set of spherical roots $\Sigma$ is the set of the sums $\alpha+\beta$ with $\alpha, \beta$ non-orthogonal simple roots.

Clearly, in order to study the multiplication of sections of line bundles and the partial order of dominant weights in the case of a model wonderful variety, we may reduce to the case where $G$ is almost simple. Moreover, if $\gamma=\sum_{\alpha \in S} a_{\alpha} \alpha \in \mathbb{Z} \Sigma$ we denote by $\operatorname{supp}_{S}(\gamma)$ the set of simple roots $\alpha$ such that $a_{\alpha} \neq 0$. We have the following lemma, whose proof is immediate.

Lemma 3.1. Let $\gamma$ be a covering difference in $\mathbb{N} \Delta$ w.r.t. $\Sigma$, then $\operatorname{supp}_{S}(\gamma)$ is a connected subset of $S$ and $\gamma$ is a covering difference for the model variety of the simply connected group associated to the root subsystem generated by $\operatorname{supp}_{S}(\gamma)$.

Therefore, we only classify the covering differences $\gamma$ such that $\operatorname{supp}_{S}(\gamma)=S$. In the following two sections, $r$ denotes the semisimple rank of the group and $\Delta=\left\{D_{1}, \ldots, D_{r}\right\}$ is the set of colors, labelled in such a way that $\omega_{D_{i}}$ is the 
fundamental weight $\omega_{i}$. For simplicity we also set $D_{i}=0$ for all $i \leqslant 0$ and for all $i>r$. Similarly, we denote $\Sigma=\left\{\sigma_{1}, \ldots, \sigma_{r-1}\right\}$ (see the following subsections for the definition of the single spherical roots $\sigma_{i}$ ), and we set $\sigma_{i}=0$ for all $i \leqslant 0$ and for all $i \geqslant r$.

For the rest of the section, $\gamma$ will be a covering difference in $\mathbb{N} \Delta$ w.r.t. $\Sigma$, and we set

$$
\gamma=\sum_{i=1}^{r-1} a_{i} \sigma_{i}=\sum_{i=1}^{r} c_{i} D_{i}
$$

For convenience we also set $a_{i}=c_{j}=0$ for all $i, j \leqslant 0$, for all $i \geqslant r$ and for all $j>r$.

3.1. Type $\mathrm{A}_{r}(r \geqslant 2)$. Let $\Sigma=\left\{\sigma_{1}, \ldots, \sigma_{r-1}\right\}$ be the set of spherical roots where $\sigma_{i}=\alpha_{i}+\alpha_{i+1}$.

Proposition 3.2. Let $\gamma \in \mathbb{N} \Sigma$ be a covering difference in $\mathbb{N} \Delta$ with $\operatorname{supp}_{S}(\gamma)=S$. Then either

(1) $r$ is even and $\gamma=\sum_{i=1}^{r / 2} \sigma_{2 i-1}=D_{1}+D_{r}$, or

(2) $r$ is odd and $\gamma=\sum_{i=1}^{r-1} \sigma_{i}=D_{2}+D_{r-1}$.

Proof. Clearly, $a_{1}>0$. Assume first that $a_{2}>0$. Take the maximum integer $k$ such that $a_{i}>0$ for all $i \leqslant k$. Then $c_{j} \geqslant 0$ for all $3 \leqslant j \leqslant k+2$ otherwise $\gamma^{-}+\sum_{i=1}^{j-2} \sigma_{i} \in \mathbb{N} \Delta$.

If $k$ were $<r-1$, then $c_{k+1}+c_{k+2}=-a_{k-1}-a_{k+3}$ would be $<0$, a contradiction. Therefore, $\operatorname{supp}_{\Sigma}(\gamma)=\Sigma$.

Now, since $\gamma^{-}+\sum_{i=1}^{r-1} \sigma_{i} \in \mathbb{N} \Delta, \gamma$ must necessarily be equal to $\sum_{i=1}^{r-1} \sigma_{i}$ which is a covering difference if and only if $r$ is odd; indeed, if $r$ is even, $\sum_{i=1}^{r / 2} \sigma_{2 i-1} \in \mathbb{N} \Delta$.

Assume now that $a_{2}=0$. Take the maximum odd integer $k$ such that $a_{i}=0$ for all even $i<k$. Then $c_{k} \geqslant 0$ otherwise $\gamma^{-}+\sum_{i=1}^{(k-1) / 2} \sigma_{2 i-1} \in \mathbb{N} \Delta$. Furthermore, $c_{k-1} \geqslant 0$ otherwise, reasoning as above, $a_{j}>0$ for all $j \geqslant k$ and $\gamma^{-}+\sum_{i=k}^{r-1} \sigma_{i} \in \mathbb{N} \Delta$.

If $k$ were $<r-1$, then $c_{k-1}+c_{k}=-a_{k+1}$ would be $<0$, a contradiction. Therefore, $a_{i}>0$ iff $i$ is odd, and $r$ is even.

Now, since $\gamma^{-}+\sum_{i=1}^{r / 2} \sigma_{2 i-1} \in \mathbb{N} \Delta, \gamma$ must necessarily be equal to $\sum_{i=1}^{r / 2} \sigma_{2 i-1}$ which is a covering difference.

3.2. Type $\mathrm{B}_{r}(r \geqslant 2)$. Let $\Sigma=\left\{\sigma_{1}, \ldots, \sigma_{r-1}\right\}$ be the set of spherical roots where $\sigma_{i}=\alpha_{i}+\alpha_{i+1}$.

Proposition 3.3. Let $\gamma \in \mathbb{N} \Sigma$ be a covering difference in $\mathbb{N} \Delta$ with $\operatorname{supp}_{S}(\gamma)=S$. Then $r$ is even and $\gamma=\sum_{i=1}^{r / 2} \sigma_{2 i-1}=D_{1}$.

Proof. Clearly, $a_{r-1}>0$. Suppose that $k<r$ is such that $r-k$ is odd and $c_{i} \leqslant 0$ for every $k<i<r$ with $r-i$ odd. Then we have the inequalities (where $a_{i}=0$ if $i \leqslant 0)$

$$
a_{r-1} \leqslant a_{r-3}-a_{r-2} \leqslant a_{r-5}-a_{r-4} \leqslant \ldots \leqslant a_{k+2}-a_{k+3} \leqslant a_{k}-a_{k+1}
$$

and it follows that $a_{j} \geqslant a_{r-1}>0$ for every $j \geqslant k$ with $r-j$ odd. In particular, $k$ must be $>0$.

Let $k$ be maximal with $r-k$ odd and $c_{k}>0$. Denote $\gamma_{0}=\sum_{i=0}^{(r-k-1) / 2} \sigma_{k+2 i}=$ $-D_{k-1}+D_{k}$; then $\gamma_{0} \leqslant \Sigma \gamma$ and $\gamma^{+}-\gamma_{0} \in \mathbb{N} \Delta$. Hence we must have $\gamma=\gamma_{0}, k=1$ and $r$ even. 
3.3. Type $C_{r}(r \geqslant 3)$. Let $\Sigma=\left\{\sigma_{1}, \ldots, \sigma_{r-1}\right\}$ be the set of spherical roots where $\sigma_{i}=\alpha_{i}+\alpha_{i+1}$.

Proposition 3.4. Let $\gamma \in \mathbb{N} \Sigma$ be a covering difference in $\mathbb{N} \Delta$ with $\operatorname{supp}_{S}(\gamma)=S$. Then $\gamma=\sum_{i=1}^{r-1} \sigma_{i}=D_{2}$.

Proof. Clearly, $a_{r-1}>0$. Let $k$ be maximal such that $c_{k}>0$. Then we have the following inequalities (for $k<r$ )

$$
a_{k-1} \geqslant a_{k} \geqslant \ldots \geqslant a_{r-1} .
$$

It follows that $a_{i}>0$ for every $k-1 \leqslant i<r$. Therefore, $\gamma^{+}-\sum_{i=k-1}^{r-1} \sigma_{i}=$ $\gamma^{+}+c_{k-2}-c_{k} \in \mathbb{N} \Delta$ and we get $k=2$ with $\gamma=\sum_{i=1}^{r-1} \sigma_{i}$.

3.4. Type $\mathrm{D}_{r}(r \geqslant 4)$. Let $\Sigma=\left\{\sigma_{1}, \ldots, \sigma_{r-1}\right\}$ be the set of spherical roots where $\sigma_{i}=\alpha_{i}+\alpha_{i+1}$ if $i<r-1$ and $\sigma_{r-1}=\alpha_{r-2}+\alpha_{r}$.

Proposition 3.5. Let $\gamma \in \mathbb{N} \Sigma$ be a covering difference in $\mathbb{N} \Delta$ with $\operatorname{supp}_{S}(\gamma)=S$. Then $r$ is odd and $\gamma=\sum_{i=1}^{(j-1) / 2} \sigma_{2 i-1}+\sum_{i=(j+1) / 2}^{(r-3) / 2} 2 \sigma_{2 i-1}+\sigma_{r-2}+\sigma_{r-1}=$ $D_{1}-D_{j-1}+D_{j}$ for $j$ odd $\leqslant r-2$.

Proof. Clearly, we have the inequalities $a_{r-2}>0$ and $a_{r-1}>0$. One has $c_{r-2} \leqslant 1$, otherwise $\gamma^{+}-\left(-2 D_{r-3}+2 D_{r-2}\right) \in \mathbb{N} \Delta$, thus $\gamma=-2 D_{r-3}+2 D_{r-2}=\sigma_{r-2}+\sigma_{r-1}$, but $\operatorname{supp}_{S}(\gamma) \neq S$. Since $c_{r-2}=-a_{r-4}+a_{r-3}+a_{r-2}+a_{r-1}$ we get $a_{r-4}>0$ and, moreover, if $c_{r-2} \leqslant 0, a_{r-4}>1$.

We go along this way step-by-step. Let $k$ be $<(r-1) / 2$. Assume $a_{r-2 i}>0$ for all $i \leqslant k$ and, moreover, if for some $j<k, c_{r-2 i} \leqslant 0$ for all $i \leqslant j$, then $a_{r-2 i-2}>1$ for all $i \leqslant j$. One has $\sum_{i=1}^{k} c_{r-2 i} \leqslant 1$, otherwise there would exist $1 \leqslant i_{1} \leqslant i_{2} \leqslant k$ such that $\gamma^{+}-\left(-D_{r-2 i_{2}-1}+D_{r-2 i_{2}}-D_{r-2 i_{1}-1}+D_{r-2 i_{1}}\right) \in \mathbb{N} \Delta$, thus $\gamma=-D_{r-2 i_{2}-1}+$ $D_{r-2 i_{2}}-D_{r-2 i_{1}-1}+D_{r-2 i_{1}}=\sum_{i=i_{1}+1}^{i_{2}} \sigma_{r-2 i}+\sum_{i=2}^{i_{1}} 2 \sigma_{r-2 i}+\sigma_{r-2}+\sigma_{r-1}$, but $\operatorname{supp}_{S}(\gamma) \neq S$. Since

$$
\sum_{i=1}^{k} c_{r-2 i}=-a_{r-2 k-2}+a_{r-2 k-1}+a_{r-2}+a_{r-1},
$$

we get $a_{r-2 k-2}>0$ and, moreover, if $c_{r-2 i} \leqslant 0$ for all $i \leqslant k, a_{r-2 k-2}>1$.

Therefore, we have that $r$ is odd, $\sum_{i=1}^{(r-1) / 2} c_{r-2 i}>1$ and $\gamma=-D_{r-2 i_{2}-1}+$ $D_{r-2 i_{2}}-D_{r-2 i_{1}-1}+D_{r-2 i_{1}}$ with $1 \leqslant i_{1} \leqslant i_{2} \leqslant(r-1) / 2 . \operatorname{Since}_{\operatorname{supp}}(\gamma)=S$, we must have $i_{2}=(r-1) / 2$.

3.5. Type $\mathrm{E}_{r}(6 \leqslant r \leqslant 8)$. Let $\Sigma=\left\{\sigma_{1}, \ldots, \sigma_{r-1}\right\}$ be the set of spherical roots where $\sigma_{1}=\alpha_{1}+\alpha_{3}, \sigma_{2}=\alpha_{2}+\alpha_{4}$ and, for $i \geqslant 3, \sigma_{i}=\alpha_{i}+\alpha_{i+1}$.

Proposition 3.6. Let $\gamma \in \mathbb{N} \Sigma$ be a covering difference in $\mathbb{N} \Delta$ with $\operatorname{supp}_{S}(\gamma)=S$. If $r=6$,

(1) either $\gamma=\sigma_{1}+\sigma_{2}+\sigma_{3}+\sigma_{4}+\sigma_{5}=D_{4}-D_{2}$

(2) or $\gamma=2 \sigma_{1}+2 \sigma_{2}+\sigma_{3}+\sigma_{4}+2 \sigma_{5}=D_{1}+D_{6}$.

If $r=7$,

(3) $\gamma=\sigma_{1}+\sigma_{2}+\sigma_{4}+\sigma_{5}+\sigma_{6}=D_{1}+D_{6}-D_{3}$.

If $r=8$,

(4) either $\gamma=\sigma_{1}+\sigma_{2}+\sigma_{3}+\sigma_{4}+2 \sigma_{5}+\sigma_{6}+\sigma_{7}=D_{6}-D_{2}$

(5) or $\gamma=2 \sigma_{1}+2 \sigma_{2}+\sigma_{3}+\sigma_{4}+2 \sigma_{5}+\sigma_{7}=D_{1}+D_{8}-D_{7}$. 
Proof. Suppose $r=6$. Clearly, $a_{1}, a_{2}, a_{5}$ are $>0$. Moreover, $c_{1}+c_{4}+c_{6}=a_{2}$.

If $c_{2}$ were $>0$, then $c_{1}, c_{4}, c_{6}$ would all be $\leqslant 0$; indeed, either $\gamma^{+}-\sigma_{2}$ or $\gamma^{+}-$ $\left(\sigma_{1}+\sigma_{2}\right)$ or $\gamma^{+}-\left(\sigma_{2}+\sigma_{5}\right)$ would be in $\mathbb{N} \Delta$, contradicting $\operatorname{supp}_{S}(\gamma)=S$ (we will use such an argument repeatedly in the rest of the proof). Therefore, $c_{2} \leqslant 0$ and $a_{3}+a_{4}>0$. By symmetry, we can assume $a_{3}>0$.

Assume $c_{4}>0$. Then $c_{3} \leqslant 0$. If $a_{4}$ were zero, $c_{2}+c_{3}$ would be equal to $a_{1}$ (which is $>0$ ). Therefore, $a_{4}>0$ and $\gamma=D_{4}-D_{2}$.

Assume now $c_{4} \leqslant 0$. We can also assume $c_{6}>0$ (by symmetry, if $a_{4}>0$ ). Then both $c_{3}$ and $c_{5}$ are $\leqslant 0$, hence $a_{4}$ is $>0$. Since $c_{2}+c_{3}+c_{4}+c_{5}=0$, all $c_{i}=0$, for $2 \leqslant i \leqslant 5$. This implies $a_{1}, a_{2}, a_{5} \geqslant 2, c_{6} \leqslant 1\left(\right.$ since $\left.\left(2 \sigma_{1}+\sigma_{2}+\sigma_{4}\right)^{+}=2 D_{1}\right)$ and $c_{1}>0$. Therefore, $\gamma=D_{1}+D_{6}$.

Suppose $r=7$. Clearly, $a_{1}, a_{2}, a_{6}$ are $>0$. Moreover, $c_{1}+c_{4}+c_{6}=a_{2}+a_{6}$.

Assume $a_{3}=0$. We get $c_{1}>0$. Then both $c_{2}$ and $c_{3}$ are $\leqslant 0$, hence $a_{4}$ is $>0$. Then $c_{7} \leqslant 0$, hence $a_{5}>0$. Moreover, $c_{4} \leqslant 0\left(\right.$ since $\left.\left(\sigma_{1}+\sigma_{2}+\sigma_{4}\right)^{+}=D_{1}+D_{4}\right)$, $c_{1} \leqslant 1\left(\right.$ since $\left.\left(2 \sigma_{1}+\sigma_{2}+\sigma_{4}\right)^{+}=2 D_{1}\right)$ and $c_{6}>0$. Therefore, $\gamma=D_{1}+D_{6}-D_{3}$.

Assume now $a_{3}>0$. If $c_{4}$ were $>0$, then both $c_{2}$ and $c_{3}$ would be $\leqslant 0$, hence $a_{4}>0$. This would imply $c_{7} \leqslant 0$, hence $a_{5}>0$, which is impossible (since $\left.\left(\sigma_{1}+\sigma_{2}+\sigma_{3}+\sigma_{4}+\sigma_{5}\right)^{+}=D_{4}\right)$. Then $c_{4} \leqslant 0$.

If $c_{1}$ were $>0$, then both $c_{2}$ and $c_{3}$ would be $\leqslant 0$, hence $a_{4}>0$. This would imply $c_{7} \leqslant 0$ hence $a_{5}>0$, and $c_{1} \leqslant 1$ (since $\left.\left(2 \sigma_{1}+\sigma_{2}+\sigma_{4}\right)^{+}=2 D_{1}\right)$ hence $c_{6}>0$, which is impossible (since $\left.\left(\sigma_{1}+\sigma_{3}+\sigma_{4}+\sigma_{5}+\sigma_{6}\right)^{+}=D_{1}+D_{6}\right)$. Then $c_{1} \leqslant 0$.

If $c_{6}$ were $>0$, we would get $c_{7} \leqslant 0, a_{5}>0$ and $c_{6} \leqslant 1$, which is impossible (since $c_{1}+c_{4}+c_{6}=a_{2}+a_{6} \geqslant 2$ ).

Suppose $r=8$. Clearly, $a_{1}, a_{2}, a_{7}$ are $>0$. Moreover, $c_{1}+c_{4}+c_{6}+c_{8}=a_{2}$.

If $c_{4}$ were $>0$, we would get $c_{2} \leqslant 0, a_{3}+a_{4}>0$, actually $a_{3}>0\left(a_{3}=0\right.$ would imply $c_{1}>0$, but also $a_{4}>0$ and $c_{1} \leqslant 0$ ), hence $c_{3} \leqslant 0, a_{4}>0, c_{4} \leqslant 1$ and $c_{1} \leqslant 0$, therefore $a_{5}>0$ (since $a_{2}+a_{4}-a_{5}=c_{1}+c_{4} \leqslant 1$ ), which is impossible (since $\left.\left(\sigma_{1}+\sigma_{2}+\sigma_{3}+\sigma_{4}+\sigma_{5}\right)^{+}=D_{4}\right)$. Then $c_{4} \leqslant 0$.

Assume $c_{6}>0$. Then $a_{5}>0$ (since $a_{6}>0$ implies $\left.c_{7} \leqslant 0\right)$, hence both $c_{2}$ and $c_{5}$ are $\leqslant 0$.

If $a_{3}$ were zero, we would get $c_{1}>0, a_{4}>0, a_{6}>0$ (since $a_{5}+a_{6}=c_{2}+c_{5} \leqslant 0$ ), which is impossible (since $\left.\left(\sigma_{1}+\sigma_{3}+\sigma_{4}+\sigma_{5}+\sigma_{6}\right)^{+}=D_{1}+D_{6}\right)$. Then $a_{3}>0$, hence $c_{3} \leqslant 0$ and $a_{4}>0$.

If $a_{6}$ were zero, since $c_{2}+c_{3}+c_{4}+c_{5}=-a_{6}$, all $c_{i}$ would be zero, for $2 \leqslant i \leqslant 5$. This would imply $a_{5} \geqslant 2$ (since $-2 a_{2}+a_{5}=c_{2}+c_{5}=0$ ), on the other hand $c_{8}$ would be $>0$, which is impossible ( since $\left.\left(\sigma_{2}+\sigma_{3}+2 \sigma_{5}+\sigma_{7}\right)^{+}=D_{6}+D_{8}\right)$. Then $a_{6}>0$, hence $c_{7} \leqslant 0$ and $a_{5} \geqslant 2$. Therefore, $\gamma=D_{6}-D_{2}$.

Assume now $c_{6} \leqslant 0$ and $c_{1}>0$. Then both $c_{2}$ and $c_{3}$ are $\leqslant 0$, hence $a_{4}>0$ and $c_{5} \leqslant 0$. If $a_{6}$ were $>0$, we would get $c_{7} \leqslant 0, a_{5}>a_{6}, a_{3}>0$ (since $-2 a_{3}+a_{5}-a_{6}=$ $\left.c_{2}+c_{5} \leqslant 0\right)$, this would imply $a_{1} \geqslant 2$ hence $c_{1} \leqslant 1\left(\right.$ since $\left.\left(2 \sigma_{1}+\sigma_{2}+\sigma_{4}\right)^{+}=2 D_{1}\right)$ and on the other hand $a_{2} \geqslant 2$ hence $c_{8}>0$ (since $c_{1}+c_{4}+c_{6}+c_{8}=a_{2}$ ), but this is impossible (since $\left.\left(2 \sigma_{1}+2 \sigma_{2}+\sigma_{3}+\sigma_{4}+2 \sigma_{5}+\sigma_{7}\right)^{+}=D_{1}+D_{8}\right)$. Then $a_{6}=0$, hence $a_{5}>0$ and $c_{8}>0$. Furthermore, since $c_{i}=0$ for all $2 \leqslant i \leqslant 5, a_{3}>0$ and $a_{1}, a_{2}, a_{5} \geqslant 2$. Therefore, $\gamma=D_{1}+D_{8}-D_{7}$.

Finally, assume both $c_{1}$ and $c_{6} \leqslant 0$. Recall that $c_{4} \leqslant 0$, therefore $c_{8} \geqslant a_{2}>0$. Futhermore, $a_{3}>0, c_{7} \leqslant 0, a_{5}>0, c_{2} \leqslant 0, c_{3} \leqslant 0$ and $a_{4}>0$. Then $a_{2} \geqslant 2$, hence $c_{8} \geqslant 2, a_{7} \geqslant 2$ and $a_{5} \geqslant 2$, which is impossible (since $\left(\sigma_{2}+\sigma_{3}+2 \sigma_{5}+2 \sigma_{7}\right)^{+}=$ $\left.2 D_{8}\right)$. 
3.6. Type $\mathrm{F}_{4}$. Let $\Sigma=\left\{\sigma_{1}, \sigma_{2}, \sigma_{3}\right\}$ be the set of spherical roots where $\sigma_{i}=\alpha_{i}+$ $\alpha_{i+1}$.

Proposition 3.7. Let $\gamma \in \mathbb{N} \Sigma$ be a covering difference in $\mathbb{N} \Delta$ with $\operatorname{supp}_{S}(\gamma)=S$. Then either

(1) $\gamma=\sigma_{1}+\sigma_{2}+2 \sigma_{3}=D_{4}$ or

(2) $\gamma=\sigma_{1}+\sigma_{3}=D_{1}+D_{4}-D_{3}$.

Proof. Clearly, $a_{1}, a_{3}>0$. Since $\sigma_{2}^{+}=D_{2}$, it follows that $c_{2} \leqslant 0$. Then $c_{4}>0$ (since $c_{2}+c_{4}=a_{1}$ ), hence $a_{3}>a_{2}$. If $a_{2}>0$, then $\gamma=D_{4}$, whereas if $a_{2}=0$, then $c_{1}>0$ and $\gamma=D_{1}+D_{4}-D_{3}$.

3.7. Type $\mathrm{G}_{2}$. Let $\Sigma=\{\sigma\}$ be the set of spherical roots where $\sigma=\alpha_{1}+\alpha_{2}$. The proof of the following proposition is trivial.

Proposition 3.8. Let $\gamma \in \mathbb{N} \Sigma$ be a covering difference in $\mathbb{N} \Delta$ with $\operatorname{supp}_{S}(\gamma)=S$. Then $\gamma=\sigma=D_{2}-D_{1}$.

\section{Projective NORMality OF MODEL WONDERFUl VARIETIES}

In this section we prove the following.

Theorem 4.1. Let $M$ be a model wonderful variety of simply connected type. The multiplication of global sections

$$
m_{\mathcal{L}, \mathcal{L}^{\prime}}: \Gamma(M, \mathcal{L}) \otimes \Gamma\left(M, \mathcal{L}^{\prime}\right) \longrightarrow \Gamma\left(M, \mathcal{L} \otimes \mathcal{L}^{\prime}\right)
$$

is surjective for all globally generated line bundles $\mathcal{L}, \mathcal{L}^{\prime} \in \operatorname{Pic}(M)$.

Following Lemma 2.3. we first classify all the low fundamental triples for the model wonderful varieties of simply connected type, and prove that these triples all satisfy the condition (2.1). As in the previous section, we can restrict ourselves to the case of an almost simple group $G$. As shown in the proof of Theorem 4.1 at the end of the section, it is enough to consider only low fundamental triples $(D, E, F)$ such that $\operatorname{supp}_{S}(D+E-F)=S$.

We keep the notation of the previous section. We denote by $H$ the stabilizer of a point $x_{0}$ in the open $G$-orbit of $M$ and by $H_{0}$ the intersection of the kernels of the multiplicative characters of $H$.

\subsection{Type $A_{r}$.}

Lemma 4.2. Let $\left(D_{p}, D_{q}, F\right)$ be a low fundamental triple with $\operatorname{supp}_{S}\left(D_{p}+D_{q}-\right.$ $F)=S$. Then $F=0$ and $p+q=r+1$. If, moreover, $r$ is odd, then $p$ and $q$ are even.

Proof. Notice that every fundamental triple is low; indeed, by Proposition 3.2 for every $\eta \in \mathbb{N} \Sigma$ covering the difference in $\mathbb{N} \Delta$ one has ht $\left(\eta^{+}\right)=2$, hence every color is minimal in $\mathbb{N} \Delta$ w.r.t. $\leqslant_{\Sigma}$. Therefore, we only need to compute the fundamental triples $\left(D_{p}, D_{q}, F\right)$ with $\operatorname{supp}_{S}\left(D_{p}+D_{q}-F\right)=S$.

Take a sequence

$$
F=F_{n}<_{\Sigma} F_{n-1}<_{\Sigma} \ldots<_{\Sigma} F_{0}=D_{p}+D_{q}
$$

such that $F_{i} \in \mathbb{N} \Delta$ and $\gamma_{i}=F_{i-1}-F_{i}$ is a covering difference for every $i \leqslant n$. By Proposition [3.2 it follows that if $\gamma_{i}^{+}=F_{i-1}=D_{s}+D_{t}$ (with $1 \leqslant s \leqslant t \leqslant r$ ) 
then $\gamma_{i}^{-}=F_{i}=D_{s-j}+D_{t+j}$ (with $j$ equal to 1 or 2$)$. Therefore, $\operatorname{supp}_{S}\left(\gamma_{n}\right)=$ $\operatorname{supp}_{S}\left(D_{p}+D_{q}-F\right)=S, F=\gamma_{n}^{-}=0$ and $q=r+1-p$.

If $r$ is odd, all the covering differences $\gamma_{i}=F_{i-1}-F_{i}$ are of the type (2) of Proposition 3.2, then $p$ is even.

Proposition 4.3. Let $r$ be odd and let $(D, E, F)$ be a low fundamental triple with $\operatorname{supp}_{S}(D+E-F)=S$. Then $s^{D+E-F} V_{F} \subset V_{D} V_{E}$.

Proof. By the previous lemma, $(D, E, F)=\left(D_{p}, D_{r+1-p}, 0\right)$ and $p$ is even (as well as $r+1-p)$.

Set $\Delta_{\text {odd }}=\left\{D_{i} \in \Delta: i\right.$ is odd $\}$; the subset $\Delta_{\text {odd }} \subset \Delta$ is distinguished and the quotient $M^{\prime}=M / \Delta_{\text {odd }}$ is a symmetric wonderful variety (with spherical roots $\left.\alpha_{2 k-1}+2 \alpha_{2 k}+\alpha_{2 k+1}\right)$.

By Corollary 1.4 together with the surjectivity of the multiplication map in the symmetric case we have

$$
\Gamma\left(M, \mathcal{L}_{D}\right) \Gamma\left(M, \mathcal{L}_{E}\right)=\Gamma\left(M^{\prime}, \mathcal{L}_{D}\right) \Gamma\left(M^{\prime}, \mathcal{L}_{E}\right)=\Gamma\left(M^{\prime}, \mathcal{L}_{D+E}\right)=\Gamma\left(M, \mathcal{L}_{D+E}\right)
$$

Proposition 4.4. Let $r$ be even and let $(D, E, F)$ be a low fundamental triple with $\operatorname{supp}_{S}(D+E-F)=S$. Then $s^{D+E-F} V_{F} \subset V_{D} V_{E}$.

Proof. By Lemma 4.2, we have $F=0$ and $V\left(\omega_{E}\right) \simeq V\left(\omega_{D}\right)^{*}$, hence $V\left(\omega_{D}\right)^{*} \otimes$ $V\left(\omega_{E}\right)^{*} \simeq \operatorname{End}\left(V\left(\omega_{D}\right)\right)$

If $r$ is even, the stabilizer $H$ of a point in the open $G$-orbit of $M$ is the normalizer in $G$ of $\operatorname{Sp}(r)$ and in particular is reductive (see [36]). Therefore, the one-dimensional $H$-submodules of $V\left(\omega_{D}\right)^{*}$ and of $V\left(\omega_{E}\right)^{*}$ associated respectively with $D$ and $E$ are dual to each other, hence we may choose the $H$-eigenvectors $h_{D} \in$ $V\left(\omega_{D}\right)^{*}$ and $h_{E} \in V\left(\omega_{E}\right)^{*} \simeq V\left(\omega_{D}\right)$ in such a way that $h_{D}\left(h_{E}\right)=1$. If we complete $h_{E}$ and $h_{D}$ to dual bases $\left\{h_{E}, v_{1}, \ldots, v_{n}\right\} \subset V\left(\omega_{D}\right)$ and $\left\{h_{D}, v_{1}^{*}, \ldots, v_{n}^{*}\right\} \subset V\left(\omega_{D}\right)^{*}$, then the identity $\operatorname{Id} \in \operatorname{End}\left(V\left(\omega_{D}\right)\right)$ is a $G$-invariant element which, as tensor, is described as follows:

$$
\mathrm{Id}=h_{D} \otimes h_{E}+\sum_{i=1}^{n} v_{i}^{*} \otimes v_{i} .
$$

Therefore, $h_{D} \otimes h_{E}$ has a non-zero projection on the isotypic component of highest weight zero and, by Lemma 1.2. we get that $s^{D+E} V_{0} \subset V_{D} V_{E}$.

\subsection{Type $\mathrm{B}_{r}$.}

Proposition 4.5. There are no low triples $(D, E, F)$ with $\sigma_{r-1} \in \operatorname{supp}_{\Sigma}(D+$ $E-F)$.

Proof. Set

$$
\begin{aligned}
\Delta^{\text {even }} & =\left\{D_{i} \in \Delta: r-i \text { is even }\right\}, \\
\Delta^{\text {odd }} & =\left\{D_{i} \in \Delta: r-i \text { is odd }\right\} .
\end{aligned}
$$

If $F<_{\Sigma} D+E$ with $\sigma_{r-1} \in \operatorname{supp}_{\Sigma}(D+E-F)$, then $\operatorname{supp}(D+E) \cap \Delta^{\text {odd }} \neq \varnothing$. Indeed, if $\operatorname{supp}(D+E)$ were included in $\Delta^{\text {even }}$, take a sequence

$$
F=F_{n}<_{\Sigma} F_{n-1}<_{\Sigma} \ldots<_{\Sigma} F_{0}=D+E
$$

of coverings in $\mathbb{N} \Delta$ : every covering difference $\gamma_{i}=F_{i-1}-F_{i}$ would necessarily be of the type (2) of Proposition 3.2 hence $\sigma_{r-1} \notin \operatorname{supp}_{\Sigma}(D+E-F)$. 
Let $k<r$ be the maximum such that $D_{k} \in \operatorname{supp}(E+F) \cap \Delta^{\text {odd }}$. Set $D+E-F=$ $\gamma=\sum_{i=1}^{r-1} a_{i} \sigma_{i}$. Since $c\left(\gamma, D_{i}\right) \leqslant 0$ for every $k<i<r$ with $r-i$ odd, as in the proof of Proposition 3.3 it follows that $a_{j} \geqslant a_{r-1}>0$ for every $j \geqslant k$ with $r-j$ odd.

Denote $\gamma_{0}=\sum_{i=0}^{(r-k-1) / 2} \sigma_{k+2 i}=-D_{k-1}+D_{k}$ and $F^{\prime}=D+E-\gamma_{0}$. Then $F^{\prime} \in \mathbb{N} \Delta$ and $F \leqslant_{\Sigma} F^{\prime}<_{\Sigma} D+E$. Set

$$
\left(D^{\prime}, E^{\prime}\right)=\left\{\begin{array}{cc}
\left(D-\gamma_{0}, E\right) & \text { if } D_{k} \in \operatorname{supp}(D), \\
\left(D, E-\gamma_{0}\right) & \text { otherwise. }
\end{array}\right.
$$

Then $D^{\prime} \leqslant_{\Sigma} D, E^{\prime} \leqslant_{\Sigma} E$ and $F \leqslant_{\Sigma} D^{\prime}+E^{\prime}<_{\Sigma} D+E$, hence $(D, E, F)$ is not a low triple.

\subsection{Type $\mathrm{C}_{r}$.}

Proposition 4.6. There are no low triples $(D, E, F)$ with $\sigma_{r-1} \in \operatorname{supp}_{\Sigma}(D+$ $E-F)$.

Proof. Let $D, E, F \in \mathbb{N} \Delta$ with $F<_{\Sigma} D+E$ and $\sigma_{r-1} \in \operatorname{supp}_{\Sigma}(D+E-F)$. Denote $k \leqslant r$ the maximum such that $D_{k} \in \operatorname{supp}(D+E)$. Reasoning as in the proof of Proposition 3.4 it follows that $\sigma_{i} \in \operatorname{supp}_{\Sigma}(D+E-F)$ for every $k-1 \leqslant i<r$. Therefore, if we set $\gamma_{0}=\sum_{i=k-1}^{r-1} \sigma_{i}=-D_{k-2}+D_{k}$ and $F^{\prime}=D+E-\gamma_{0}$, then $F^{\prime} \in \mathbb{N} \Delta$ and $F \leqslant_{\Sigma} F^{\prime}<D+E$. Set

$$
\left(D^{\prime}, E^{\prime}\right)=\left\{\begin{array}{cc}
\left(D-\gamma_{0}, E\right) & \text { if } D_{k} \in \operatorname{supp}(E) \\
\left(D, E-\gamma_{0}\right) & \text { otherwise }
\end{array}\right.
$$

Then $D^{\prime} \leqslant_{\Sigma} D, E^{\prime} \leqslant_{\Sigma} E$ and $F \leqslant_{\Sigma} D^{\prime}+E^{\prime}<_{\Sigma} D+E$, hence $(D, E, F)$ is not a low triple.

\subsection{Type $\mathrm{D}_{r}$.}

Lemma 4.7. Let $\left(D_{p}, D_{q}, F\right)$ be a low fundamental triple with $\operatorname{supp}_{S}\left(D_{p}+D_{q}-\right.$ $F)=S$. Then $p, q, r$ are odd, $p, q \leqslant r-2$ and either

(1) $p+q \leqslant r-1$ with $F=D_{p+q-2}$ or

(2) $p+q=r+1$ with $F=D_{r-1}+D_{r}$.

Proof. Notice that, as in type A, every fundamental triple is low. Therefore, we only need to compute the fundamental triples $\left(D_{p}, D_{q}, F\right)$ with $\operatorname{supp}_{S}\left(D_{p}+D_{q}-F\right)=S$. Assume $p \leqslant q$.

Take a sequence

$$
F=F_{n}<_{\Sigma} F_{n-1}<_{\Sigma} \ldots<_{\Sigma} F_{0}=D_{p}+D_{q}
$$

such that $F_{i} \in \mathbb{N} \Delta$ and $\gamma_{i}=F_{i-1}-F_{i}$ is a covering difference for every $i \leqslant n$. Recall the classification of covering differences of Propositions 3.2 and 3.5 .

(1) $p, q \leqslant r-2$. If $q$ were equal to $r-1$ then $(r-1)-p$ should be non-zero and odd, thus $\gamma_{1}=-D_{p-1}+D_{p}+D_{r-1}-D_{r}$ and $F_{1}=D_{p-1}+D_{r}$, which is impossible since the distance between the vertices $r$ and $p-1$ is even. By symmetry, the same argument works if $q=r$.

(2) $q-p$ is even. If $q-p$ were odd, there would exist $i$ such that $F_{i}$ is $D_{p^{\prime}}+2 D_{q^{\prime}}$ with $q^{\prime}$ equal to $r-1$ or $r$ and $(r-1)-p^{\prime}$ even, which is impossible.

(3) $r-p$ is even (as well as $r-q$ ). Here again if $r-p$ and $r-q$ were odd, there would exist $i$ such that $F_{i}$ is $D_{p^{\prime}}+2 D_{q^{\prime}}$ with $q^{\prime}$ equal to $r-1$ or $r$ and $(r-1)-p^{\prime}$ even. 
Therefore, there exists $i$ such that $\gamma_{j}$ is of the type (2) of Proposition 3.2 for every $j<i$ and $\gamma_{i}$ is either of type (1) of Proposition 3.2 or of type D.

In the first case, $F_{i-2}=D_{p^{\prime}}+D_{q^{\prime}}$ with $q^{\prime}=r-2$ and $p^{\prime}+q^{\prime}=p+q$, $F_{i-1}=D_{p^{\prime}-2}+2 D_{q^{\prime \prime}}$ where $q^{\prime \prime}$ is equal to $r-1$ or $r$. Then necessarily $p^{\prime}-2=1$ and $F_{i}=F_{n}=D_{r-1}+D_{r}$.

In the second case, $F_{i-1}=D_{p^{\prime}}+D_{q^{\prime}}$ with $p^{\prime}+q^{\prime}=p+q$ and $F_{i}=D_{p^{\prime}-1}+D_{q^{\prime}-1}$. Then $\gamma_{j}$ is of the type (2) of Proposition 3.2 for every $j>i$ until $F_{n-1}=D_{p^{\prime}-1-2 k}+$ $D_{q^{\prime}-1+2 k}$ where $p^{\prime}-1-2 k=2(k=n-1-i)$ and $F_{n}$ is equal to either $D_{q^{\prime}-1+2 k+2}$ if $q^{\prime}-1+2 k \leqslant r-5$ or $D_{r-1}+D_{r}$ if $q^{\prime}-1+2 k=r-3$.

Proposition 4.8. Let $(D, E, F)$ be a low fundamental triple with $\operatorname{supp}_{S}(D+E-$ $F)=S$, then $s^{D+E-F} V_{F} \subset V_{D} V_{E}$.

Proof. We need an explicit computation.

Denote by $U=\mathbb{C}^{2 r}$ the first fundamental representation of $G$ (that is, the standard representation of $\mathrm{SO}(2 r))$ and fix $b \in \mathrm{S}^{2} U$ a $G$-invariant non-degenerate symmetric 2-form. If $W \subset U$ is a maximal isotropic subspace, we then get a decomposition $U=W \oplus W^{*}$. Fix a non-zero vector $e_{0} \in W$ and consider the corresponding decomposition $U=V \oplus \mathbb{C} e_{0} \oplus V^{*} \oplus \mathbb{C} e_{0}^{*}$, where $V \subset W$ is a complement of the line $\mathbb{C} e_{0}$ and where $e_{0}^{*} \in W^{*}$ is defined by $e_{0}^{*}\left(e_{0}\right)=1,\left.e_{0}^{*}\right|_{V}=0$.

Then (see [36]) the Lie algebra $\mathfrak{h}_{0}$ of $H_{0}$ can be described as

$$
\mathfrak{h}_{0}=\mathfrak{s p}(V) \oplus V^{*} \oplus \operatorname{Skew}\left(V, V^{*}\right),
$$

where $\operatorname{Skew}\left(V, V^{*}\right) \subset \operatorname{Hom}\left(V, V^{*}\right)$ denotes the subspace of skew-symmetric linear maps and where $\mathfrak{h}_{0}$ is embedded in $\mathfrak{s o}(U)$ as follows (here we denote by $u=$ $\left(v, \lambda e_{0}, \psi, \mu e_{0}^{*}\right)$ a generic element in $\left.U=V \oplus \mathbb{C} e_{0} \oplus V^{*} \oplus \mathbb{C} e_{0}^{*}\right)$ :

- if $f \in \mathfrak{s p}(V)$, then $f(u)=f(v)+f \cdot \psi$,

- if $\phi \in V^{*}$, then $\phi(u)=\phi(v) e_{0}-(\lambda+\mu) \phi+\phi(v) e_{0}^{*}$,

- if $\Phi \in \operatorname{Skew}\left(V, V^{*}\right)$, then $\Phi(u)=\Phi(v)$.

As already mentioned at the beginning of Section 3 every simple $G$-module possesses a unique $\mathfrak{h}_{0}$-invariant element, up to scalars. In particular, if we denote $h_{1}=e_{0}-e_{0}^{*} \in U$ and $h_{2} \in \Lambda^{2} V^{*}$ a $\mathfrak{s p}(V)$-invariant non-degenerate 2-form, then $h_{1} \in U^{\mathfrak{h}_{0}}$ and $h_{2} \in\left(\Lambda^{2} U\right)^{\mathfrak{h}_{0}}$. In this way we may describe the $\mathfrak{h}_{0}$-invariant vectors in every exterior power $\Lambda^{i} U$ with $i \leqslant r-1$. Indeed, set

$$
h_{i}=\left\{\begin{array}{cl}
h_{2}^{\wedge k} & \text { if } i=2 k \text { is even } \\
h_{1} \wedge h_{2}^{\wedge k} & \text { if } i=2 k+1 \text { is odd }
\end{array}\right.
$$

then $h_{i} \in\left(\Lambda^{i} U\right)^{\mathfrak{h}_{0}}$.

Set $\omega_{0}=0$ and recall that if $i \geqslant 0$ then

$$
\Lambda^{i} U=\left\{\begin{array}{cl}
V\left(\omega_{i}\right) & \text { if } i \leqslant r-2, \\
V\left(\omega_{r-1}+\omega_{r}\right) & \text { if } i=r-1 .
\end{array}\right.
$$

To conclude the proof, by Lemma 1.2 we only need to show that, if $i, j$ are odd integers with $i+j \leqslant r+1$, then there exists an equivariant projection $\pi: \wedge^{i} U \otimes$ $\Lambda^{j} U \longrightarrow \wedge^{i+j-2} U$ such that $\pi\left(h_{i} \otimes h_{j}\right) \neq 0$. Define $\pi$ as follows:

$\pi\left(\left(u_{1} \wedge \cdots \wedge u_{i}\right) \otimes\left(w_{1} \wedge \cdots \wedge w_{j}\right)\right)=\sum_{h, k}(-1)^{h+k} b\left(u_{h}, w_{k}\right) u_{1} \wedge \cdots \wedge \hat{u_{h}} \wedge \cdots \wedge \hat{w_{k}} \wedge \cdots \wedge w_{j}$. 
Suppose that $i, j$ are odd with $i+j \leqslant r+1$ and set $k=(i+j-2) / 2$. Notice that $\pi\left(h_{i} \otimes h_{j}\right)=b\left(h_{1}, h_{1}\right) h_{2}^{\wedge k}+q$, where $q \in \Lambda^{i+j-2} U$ is linearly independent with $h_{2}^{\wedge k}$; since $b\left(h_{1}, h_{1}\right) \neq 0$ and since $h_{2}^{\wedge k} \neq 0$, it follows then $\pi\left(h_{i} \otimes h_{j}\right) \neq 0$.

\subsection{Type $\mathrm{E}_{r}$.}

Lemma 4.9. The low fundamental triples $(D, E, F)$ with $\operatorname{supp}_{S}(D+E-F)=S$ are the following:

- If $r=6:\left(D_{1}, D_{3}, D_{2}\right)\left(D_{1}, D_{5}, D_{3}\right)\left(D_{1}, D_{6}, 0\right)\left(D_{3}, D_{6}, D_{5}\right)\left(D_{5}, D_{6}, D_{2}\right)$,

- If $r=7:\left(D_{1}, D_{6}, D_{3}\right)\left(D_{6}, D_{6}, D_{2}+D_{7}\right)$,

- If $r=8:\left(D_{1}, D_{1}, D_{2}\right)\left(D_{1}, D_{5}, 2 D_{2}\right)\left(D_{1}, D_{7}, D_{3}\right)\left(D_{1}, D_{8}, D_{7}\right)\left(D_{3}, D_{8}, D_{5}\right)$ $\left(D_{5}, D_{8}, D_{2}+D_{7}\right)\left(D_{7}, D_{8}, D_{2}\right)$.

By making use of Proposition 3.6. the proof of this lemma is a quite long but trivial case-by-case computation, which we do not report here.

Proposition 4.10. Let $(D, E, F)$ be a low fundamental triple with $\operatorname{supp}_{S}(D+E-$ $F)=S$, then $s^{D+E-F} V_{F} \subset V_{D} V_{E}$.

Let us first deal with the triple $\left(D_{1}, D_{6}, 0\right)$ of $\mathrm{E}_{6}$. The set $\Delta_{0}=\left\{D_{2}, D_{3}, D_{4}, D_{5}\right\}$ is distinguished and the quotient $M^{\prime}=M / \Delta_{0}$ is a symmetric wonderful variety (with spherical roots $\left\{2 \alpha_{1}+\alpha_{2}+2 \alpha_{3}+2 \alpha_{4}+\alpha_{5}, \alpha_{2}+\alpha_{3}+2 \alpha_{4}+2 \alpha_{5}+2 \alpha_{6}\right.$ ). Therefore, we can conclude by Corollary 1.4 and the surjectivity of the multiplication map in the symmetric case.

For all the other triples of Lemma 4.9 we have to use Lemma 1.2. Since the dimension of the involved representations is quite high, we have used the computer and, more precisely, GAP [23], a software for computations which contains built-in functions to construct and deal with representations of simple Lie algebras (see also [21]).

Although the dimension of some of the involved representations is very high, we have succeeded to make the computation accessible with a currently available home computer. A quite convenient tool is the quadratic Casimir operator $c$, which acts as the scalar $(\lambda+2 \rho, \lambda)$ on every irreducible representation $V(\lambda)$. Let $(D, E, F)$ be a low fundamental triple, once the irreducible representations $V_{D}^{*}$ and $V_{E}^{*}$ are constructed, and the vectors $h_{D}$ and $h_{E}$ are explicitly found, there is no need to construct the whole tensor product $V_{D}^{*} \otimes V_{E}^{*}$ in order to project $h_{D} \otimes h_{E}$ onto the eigenspace of $c$ relative to $\left(\omega_{F}^{*}+2 \rho, \omega_{F}^{*}\right)$. Moreover, in all our cases there exists only one isotypical component in $V_{D}^{*} \otimes V_{E}^{*}$ of eigenvalue $\left(\omega_{F}^{*}+2 \rho, \omega_{F}^{*}\right)$ with respect to $c$.

Sometimes for $\mathrm{E}_{8}$ the dimension is so high that it is even quite costly to construct the irreducible representation $V_{D}^{*}$ itself $\left(V_{D_{5}}\right.$ has dimension $\left.146,325,270\right)$. Here we use a further escamotage. The set $\Delta_{0}=\left\{D_{1}, D_{4}, D_{6}, D_{8}\right\}$ is distinguished and the quotient $M^{\prime}=M / \Delta_{0}$ is the parabolic induction of a symmetric wonderful SL(8)variety with spherical roots $\left\{\alpha_{1}+2 \alpha_{3}+\alpha_{4}, \alpha_{4}+2 \alpha_{5}+\alpha_{6}, \alpha_{6}+2 \alpha_{7}+\alpha_{8}\right\}$. This means that, if $D$ equals $D_{3}, D_{5}$ or $D_{7}$, then $h_{D}$ can be choosen to be the $\operatorname{Sp}(8)$ invariant vector in the simple $\mathrm{SL}(8)$-submodule $W_{D} \subset V_{D}$ of highest weight $\omega_{D}$. Furthermore, $V_{D_{1}}$ and $V_{D_{8}}$ are still accessible (the former has dimension 3875 and the latter is the adjoint representation), $V_{D_{7}} \subset \Lambda^{2} V_{D_{8}}, V_{D_{5}} \subset \Lambda^{4} V_{D_{8}}, V_{D_{3}} \subset \Lambda^{2} V_{D_{1}}$, and respectively $W_{D_{7}} \subset \Lambda^{2} W_{D_{8}}, W_{D_{5}} \subset \Lambda^{4} W_{D_{8}}, W_{D_{3}} \subset \Lambda^{2} W_{D_{1}}$. Therefore, the vectors $h_{D}$ can be explicitly determined if $D$ equals $D_{1}, D_{3}, D_{5}, D_{7}$ or $D_{8}$, and notice that this is enough to treat all the above triples. 


\subsection{Type $\mathrm{F}_{4}$.}

Lemma 4.11. The only low fundamental triple $(D, E, F)$ with $\operatorname{supp}_{S}(D+E-F)=$ $S$ is $\left(D_{1}, D_{4}, D_{3}\right)$.

The proof is trivial, after Proposition 3.7

Proposition 4.12. $s^{D_{1}+D_{4}-D_{3}} V_{D_{3}} \subset V_{D_{1}} V_{D_{4}}$.

This can easily be checked via computer (as explained above).

\subsection{Type $\mathrm{G}_{2}$.}

Proposition 4.13. There are no low triples $(D, E, F)$ with $F \neq D+E$.

The proof is trivial.

4.8. Projective normality of model wonderful varieties. We are now ready to prove that the multiplication of sections on a model wonderful variety of simply connected type is surjective.

A localization of a wonderful variety $M$ is a $G$-stable subvariety of $M$, which is a wonderful $G$-variety by itself. Notice that we have a bijective correspondence between localizations of $M$ and subsets of $\Sigma$. More precisely, for all subsets $\Sigma^{\prime}$ of $\Sigma$, the intersection of the boundary divisors $M^{\sigma}$ for $\sigma \in \Sigma^{\prime}$ gives a wonderful variety with $\Sigma \backslash \Sigma^{\prime}$ as a set of spherical roots.

Proof of Theorem 4.1. By the classification of the covering differences given in the previous section, it follows that the model wonderful varieties of simply connected type satisfy the property (2-ht). Hence by Lemma 2.3 it is enough to show the inclusion $s^{D+E-F} V_{F} \subset V_{D} V_{E}$ for all the low fundamental triples $(D, E, F)$. Here we only need to show that we can reduce to the low fundamental triples $(D, E, F)$ such that $\operatorname{supp}_{S}(D+E-F)=S$, whose case has been proved above in this section.

Let $(D, E, F)$ be a low fundamental triple, denote $\gamma=D+E-F$ and $S^{\prime}=$ $\operatorname{supp}_{S}(\gamma)$ and suppose that $S^{\prime} \neq S$. Let $Q$ be the parabolic subgroup associated to $S^{\prime}$ and set $G^{\prime}=Q / R_{Q}$. Since $(D, E, F)$ is a low triple, it follows that $S^{\prime}$ is connected, hence $G^{\prime}$ is an almost simple group. Consider the localization $M^{\prime}$ of $M$ with $\Sigma^{\prime}=\left\{\sigma \in \Sigma: \operatorname{supp}_{S}(\sigma) \subset S^{\prime}\right\}$ as a set of spherical roots. Then $M^{\prime}$ is the parabolic induction of the model wonderful $G^{\prime}$-variety $N$ of simply connected type.

The restrictions $\operatorname{Pic}(M) \longrightarrow \operatorname{Pic}\left(M^{\prime}\right)$ and $\operatorname{Pic}\left(M^{\prime}\right) \longrightarrow \operatorname{Pic}(N)$ identify $\operatorname{Pic}(N)$ with a sublattice of $\operatorname{Pic}(M)$. More precisely, if $\widetilde{\Delta} \subset \Delta$ is the set of colors $\widetilde{D}$ such that $\omega_{\widetilde{D}}=\omega_{\alpha}$ for some $\alpha \in S^{\prime}$, then $\operatorname{Pic}(N)$ is identified with $\mathbb{Z} \widetilde{\Delta}$. Moreover, an element of $\mathbb{Z} \widetilde{\Delta}$ induces a globally generated line bundle on $N$ if and only if its coefficients are non-negative. If $\widetilde{D} \in \mathbb{N} \widetilde{\Delta}$, set $W_{\widetilde{D}}=V_{\widetilde{D}}^{U_{Q}}$.

Notice that $D, E \in \widetilde{\Delta}$ and consider the difference $\widetilde{F}=D+E-\gamma \in \mathbb{Z} \widetilde{\Delta}$ (where $\gamma$ is regarded as sum of spherical roots of $N$ hence as an element of $\mathbb{Z} \widetilde{\Delta}$ ). Since $(D, E, F)$ is a low triple in $\mathbb{N} \Delta$, it follows that $(D, E, \widetilde{F})$ is a low triple in $\mathbb{N} \widetilde{\Delta}$. Since $\operatorname{supp}_{S^{\prime}}(\gamma)=S^{\prime}$, we have that $s^{\gamma} W_{\widetilde{F}} \subset W_{D} W_{E}$, therefore Proposition 1.6 implies that $s^{\gamma} V_{F} \subset V_{D} V_{E}$ with respect to the multiplication map $m_{D, E}^{\prime}: \Gamma\left(M^{\prime}, \mathcal{L}_{D}\right) \otimes$ $\Gamma\left(M^{\prime}, \mathcal{L}_{E}\right) \longrightarrow \Gamma\left(M^{\prime}, \mathcal{L}_{D+E}\right)$. This concludes the proof, since $m_{D, E}^{\prime}$ is just the restriction of the multiplication map $m_{D, E}: \Gamma\left(M, \mathcal{L}_{D}\right) \otimes \Gamma\left(M, \mathcal{L}_{E}\right) \longrightarrow \Gamma\left(M, \mathcal{L}_{D+E}\right)$. 
Let $M$ be a wonderful variety and let $N$ be a quotient of $M$. Then the pullback of line bundles identifies $\operatorname{Pic}(N)$ with a sublattice of $\operatorname{Pic}(M)$ and a line bundle $\mathcal{L} \in \operatorname{Pic}(N)$ is generated by global sections if and only if its pull-back (which we still denote by $\mathcal{L})$ is. Moreover, by Corollary 1.4 we have $\Gamma(N, \mathcal{L})=\Gamma(M, \mathcal{L})$. It follows that if $\mathcal{L}, \mathcal{L}^{\prime} \in \operatorname{Pic}(N)$ are generated by global sections and if the multiplication $\Gamma(M, \mathcal{L}) \otimes \Gamma\left(M, \mathcal{L}^{\prime}\right) \longrightarrow \Gamma\left(M, \mathcal{L} \otimes \mathcal{L}^{\prime}\right)$ is surjective, then the multiplication $\Gamma(N, \mathcal{L}) \otimes$ $\Gamma\left(N, \mathcal{L}^{\prime}\right) \longrightarrow \Gamma\left(N, \mathcal{L} \otimes \mathcal{L}^{\prime}\right)$ is also surjective.

Now let $N$ be a localization of $M$ and let $\mathcal{L}, \mathcal{L}^{\prime} \in \operatorname{Pic}(M)$ be generated by global sections. Then the restriction of sections to $N$ is surjective, therefore if the multiplication $\Gamma(M, \mathcal{L}) \otimes \Gamma\left(M, \mathcal{L}^{\prime}\right) \longrightarrow \Gamma\left(M, \mathcal{L} \otimes \mathcal{L}^{\prime}\right)$ is surjective, the multiplication $\Gamma\left(N,\left.\mathcal{L}\right|_{N}\right) \otimes \Gamma\left(N,\left.\mathcal{L}^{\prime}\right|_{N}\right) \longrightarrow \Gamma\left(N,\left.\left.\mathcal{L}\right|_{N} \otimes \mathcal{L}^{\prime}\right|_{N}\right)$ is also surjective.

If, moreover, $M$ is a model wonderful variety of simply connected type, then by the description of the restriction $\omega: \operatorname{Pic}(M) \longrightarrow \mathcal{X}(T)$ (see [42, Lemma 30.24]) we get an isomorphism $\operatorname{Pic}(M) \simeq \mathcal{X}(T)$, which identifies the semigroup of globally generated (resp. ample) line bundles on $M$ with $\mathcal{X}(T)^{+}$(resp. with the semigroup of regular dominant weights). If $N$ is any localization of $M$, then $\omega$ factors through $N$ and we get an isomorphism $\operatorname{Pic}(M) \simeq \operatorname{Pic}(N)$, which identifies the globally generated (resp. the ample) line bundles on $M$ and on $N$. Up to replacing $\mathcal{X}(T)$ with some sublattice generated by fundametal weights, the same is true whenever $M^{\prime}$ is the quotient of a localization of a model wonderful variety of simply connected type and $N^{\prime}$ is a localization of $M^{\prime}$. Therefore, proceeding inductively, we get the following corollary of Theorem 4.1,

Corollary 4.14. Let $N$ be a wonderful variety obtained from a model wonderful variety of simply connected type via operations of localization and quotient by distinguished sets of colors. Then the multiplication map

$$
m_{\mathcal{L}, \mathcal{L}^{\prime}}: \Gamma(N, \mathcal{L}) \otimes \Gamma\left(N, \mathcal{L}^{\prime}\right) \longrightarrow \Gamma\left(N, \mathcal{L} \otimes \mathcal{L}^{\prime}\right)
$$

is surjective for all globally generated line bundles $\mathcal{L}, \mathcal{L}^{\prime} \in \operatorname{Pic}(N)$.

\section{Projective NORMALITY OF COMODEL WONDERFUl VARIETIES}

Motivated by an application which we illustrate below in Section 8 , here we study the surjectivity of the multiplication of sections in another class of wonderful varieties, which we call comodel.

Let $G$ be simply connected of simply-laced type, and fix $T$ and $B$ as usual. Let $M$ be a model wonderful variety of $G$, with set of colors $\Delta$ (in bijection with the set of fundamental weights), set of spherical roots $\Sigma$ and Cartan pairing $c: \Delta \times \Sigma \rightarrow \mathbb{Z}$. Let $G^{\vee}$ be a simply connected group whose root system is isomorphic to $\Phi_{\Sigma}$, the root system generated by $\Sigma$. Once fixed $T^{\vee}$ and $B^{\vee}$, its set of simple roots $S^{\vee}$ is thus in bijective correspondence with $\Sigma$. From [5] and [9] it follows that:

Theorem 5.1. There exists a wonderful $G^{\vee}$-variety $M^{\vee}$ whose set of spherical roots $\Sigma^{\vee}$ is equal to the set of simple roots $S^{\vee}$ of $G^{\vee}$, its set of colors $\Delta^{\vee}$ is in bijective correspondence with $\Delta$ and, under these correspondences, its Cartan pairing $c^{\vee}: \Delta^{\vee} \times \Sigma^{\vee} \rightarrow \mathbb{Z}$ equals the Cartan pairing $c$ of the model wonderful $G$ variety $M$.

We say that the wonderful variety $M^{\vee}$ of the previous theorem is a comodel wonderful variety of $G^{\vee}$, and the type of $G$ is called the cotype of $M^{\vee}$. 
Forgetting the model wonderful variety, in the following $M$ is a comodel wonderful variety of $G, H$ denotes the stabilizer of a point $x_{0}$ in the open orbit of $M$, and $H_{0}$ the kernel of its multiplicative characters; $\mathfrak{h}_{0}$ denotes the Lie algebra of $H_{0}$ in the Lie algebra $\mathfrak{g}$ of $G$.

The comodel wonderful varieties correspond to the following cases in [5], an explicit description of the corresponding subgroups $H$ can be found in [9].

- Cotype $A_{2 m}$ : type $A_{m-1} \times A_{m}$, case S-5.

- Cotype $A_{2 m+1}$ : type $A_{m} \times A_{m}$, case $S-4$.

- Cotype $\mathrm{D}_{2 m}$ : type $\mathrm{A}_{m-1} \times \mathrm{D}_{m}$, case S-10.

- Cotype $D_{2 m+1}$ : type $A_{m-1} \times D_{m+1}$, case $S-11$.

- Cotype $E_{6}$ : type $A_{5}$, case S-50.

- Cotype $E_{7}$ : type $A_{6}$, case S-49.

- Cotype $\mathrm{E}_{8}$ : type $\mathrm{D}_{7}$, case S-58.

In this section we prove the following.

Theorem 5.2. Let $M$ be a comodel wonderful variety and let $\mathcal{L}, \mathcal{L}^{\prime}$ be line bundles generated by global sections. Then the multiplication map $m_{\mathcal{L}, \mathcal{L}^{\prime}}$ is surjective.

Let $M$ be a comodel wonderful variety, we enumerate the colors of $M$ as in the corresponding model wonderful variety. Similarly, we denote by $h_{i} \in V_{D_{i}}^{*}$ the $H$-semi-invariant associated to $D_{i}$. This vector is invariant under $H_{0}$.

More explicitly, set the map $\omega: \operatorname{Pic}(M) \longrightarrow \mathcal{X}(T)$ as follows (if the Dynkin diagram of $G$ has two connected components, we distinguish with a superscript the fundamental weights coming from the different components).

- Cotype $\mathrm{A}_{2 m}: \omega\left(D_{1}\right)=\omega_{1}^{\prime}, \omega\left(D_{2 i}\right)=\omega_{i}+\omega_{i}^{\prime}$ for $i<m, \omega\left(D_{2 i-1}\right)=\omega_{i-1}+\omega_{i}^{\prime}$ for $i>1, \omega\left(D_{2 m}\right)=\omega_{m}^{\prime}$.

- Cotype $A_{2 m+1}: \omega\left(D_{1}\right)=\omega_{1}, \omega\left(D_{2 i}\right)=\omega_{i}+\omega_{i}^{\prime}, \omega\left(D_{2 i-1}\right)=\omega_{i}+\omega_{i-1}^{\prime}$ for $1<i<m+1, \omega\left(D_{2 m+1}\right)=\omega_{m}^{\prime}$.

- Cotype $D_{2 m}: \omega\left(D_{1}\right)=\omega_{1}, \omega\left(D_{2 i}\right)=\omega_{i}+\omega_{i}^{\prime}$ for $i<m-1, \omega\left(D_{2 i-1}\right)=\omega_{i}+$ $\omega_{i-1}^{\prime}$ for $1<i<m, \omega\left(D_{2 m-2}\right)=\omega_{m-1}+\omega_{m-1}^{\prime}+\omega_{m}^{\prime}, \omega\left(D_{2 m-1}\right)=\omega_{m-1}^{\prime}$, $\omega\left(D_{2 m}\right)=\omega_{m}^{\prime}$.

- Cotype $\mathrm{D}_{2 m+1}: \omega\left(D_{1}\right)=\omega_{1}^{\prime}, \omega\left(D_{2 i}\right)=\omega_{i}+\omega_{i}^{\prime}$ for $i<m, \omega\left(D_{2 i-1}\right)=$ $\omega_{i-1}+\omega_{i}^{\prime}$ for $1<i<m, \omega\left(D_{2 m-1}\right)=\omega_{m-1}+\omega_{m}^{\prime}+\omega_{m+1}^{\prime}, \omega\left(D_{2 m}\right)=\omega_{m}^{\prime}$, $\omega\left(D_{2 m+1}\right)=\omega_{m+1}^{\prime}$.

- Cotype $\mathrm{E}_{6}: \omega\left(D_{1}\right)=\omega_{2}, \omega\left(D_{2}\right)=\omega_{3}, \omega\left(D_{3}\right)=\omega_{2}+\omega_{5}, \omega\left(D_{4}\right)=\omega_{1}+\omega_{3}+$ $\omega_{5}, \omega\left(D_{5}\right)=\omega_{1}+\omega_{4}, \omega\left(D_{6}\right)=\omega_{4}$.

- Cotype $\mathrm{E}_{7}: \omega\left(D_{1}\right)=\omega_{3}, \omega\left(D_{2}\right)=\omega_{4}, \omega\left(D_{3}\right)=\omega_{3}+\omega_{6}, \omega\left(D_{4}\right)=\omega_{2}+\omega_{4}+$ $\omega_{6}, \omega\left(D_{5}\right)=\omega_{2}+\omega_{5}, \omega\left(D_{6}\right)=\omega_{1}+\omega_{5}, \omega\left(D_{7}\right)=\omega_{1}$.

- Cotype $\mathrm{E}_{8}: \omega\left(D_{1}\right)=\omega_{3}, \omega\left(D_{2}\right)=\omega_{4}, \omega\left(D_{3}\right)=\omega_{3}+\omega_{6}, \omega\left(D_{4}\right)=\omega_{2}+\omega_{4}+$ $\omega_{6}, \omega\left(D_{5}\right)=\omega_{2}+\omega_{5}, \omega\left(D_{6}\right)=\omega_{1}+\omega_{5}, \omega\left(D_{7}\right)=\omega_{1}+\omega_{7}, \omega\left(D_{8}\right)=\omega_{7}$.

Since the Cartan pairing of the comodel wonderful varieties is the same as that of the model varieties, the classification of the covering differences is also the same and the property (2-ht) holds. As shown in Section [5.6, in order to apply Lemma 2.3 it is enough to test the surjectivity on the same low fundamental triples arising in Section 4 in the model case.

In the computations below we use the following conventions. We denote by $e_{1}, \ldots, e_{n}$ the standard basis of $V=\mathbb{C}^{n}$ and by $\varphi_{1}, \ldots, \varphi_{n}$ the dual basis. We also denote by $e_{i_{1} i_{2} \ldots i_{k}}$ the vector $e_{i_{1}} \wedge e_{i_{2}} \wedge \cdots \wedge e_{i_{k}} \in \Lambda^{k} V$ and similarly for $\varphi_{i_{1} i_{2} \ldots i_{k}}$. On $V \oplus V^{*}$ the symmetric bilinear form $(u, \varphi) \cdot(v, \psi)=\varphi(v)+\psi(u)$ is 
defined. A pairing between $\Lambda^{k} V$ and $\Lambda^{n-k} V$ is defined by $\langle x, y\rangle=\frac{x \wedge y}{e_{1 \ldots n}}$ and we denote by $\gamma_{k}: \Lambda^{k} V \longrightarrow \Lambda^{n-k} V^{*}$ the associated map. We also identify $V_{1} \otimes V_{2}$ with $\operatorname{Hom}\left(V_{1}^{*}, V_{2}\right)$ in the usual way, and if $V_{1}=V_{2}=V$ we identify $\mathrm{S}^{2} V$ and $\wedge^{2} V$ with symmetric and antisymmetric linear maps in $\operatorname{Hom}\left(V^{*}, V\right)$.

Starting with the vector space $V$ we construct a new vector space $W=V \oplus V^{*} \oplus Z$ as the direct sum of $V, V^{*}$ and another piece $Z$ that is zero, one- or two-dimensional. In particular, the dual of $W$ can be identified with $V^{*} \oplus V \oplus Z^{*}$. Once a basis of $Z$ is fixed, say $z_{1}, \ldots, z_{t}$, we denote the dual basis of $e_{1}, \ldots, e_{n}, \varphi_{1}, \ldots, \varphi_{n}, z_{1}, \ldots, z_{t}$ by $e_{1}^{*}, \ldots, e_{n}^{*}, \varphi_{1}^{*}, \ldots, \varphi_{n}^{*}, z_{1}^{*}, \ldots, z_{t}^{*}$.

For a vector space $U$ we have contraction maps $\kappa_{U}^{i j}: \Lambda^{i} U \otimes \Lambda^{j} U^{*} \longrightarrow \Lambda^{i-1} U \otimes$ $\Lambda^{j-1} U^{*}$ given by

$$
\begin{aligned}
& \kappa_{U}^{i j}\left(u_{1} \wedge \cdots \wedge u_{i} \otimes \varphi_{1} \wedge \cdots \wedge \varphi_{j}\right) \\
& \quad=\sum_{k, l}(-1)^{k+l} \varphi_{l}\left(u_{k}\right) u_{1} \wedge \cdots \wedge \hat{u}_{k} \wedge \cdots \wedge u_{i} \otimes \varphi_{1} \wedge \cdots \wedge \hat{\varphi}_{l} \wedge \cdots \wedge \varphi_{j} .
\end{aligned}
$$

In particular, we set $\kappa_{U}=\kappa_{U}^{21}$.

Similarly, for a vector space $U$ with a symmetric bilinear form (, ) we have $\tilde{\kappa}_{U}^{i j}: \Lambda^{i} U \otimes \Lambda^{j} U \longrightarrow \Lambda^{i+j-2} U$ such that

$$
\begin{aligned}
& \tilde{\kappa}_{U}^{i j}\left(u_{1} \wedge \cdots \wedge u_{i} \otimes v_{1} \wedge \cdots \wedge v_{j}\right) \\
& \quad=\sum_{k, l}(-1)^{k+l}\left(u_{k}, v_{l}\right) u_{1} \wedge \cdots \wedge \hat{u}_{k} \wedge \cdots \wedge u_{i} \wedge v_{1} \wedge \cdots \wedge \hat{v}_{l} \wedge \cdots \wedge v_{j} .
\end{aligned}
$$

5.1. Cotype $\mathrm{A}_{r}$. We test the triples of Lemma 4.2. $\left(D_{p}, D_{r+1-p}, 0\right)$ where, if $r$ is odd, $p$ is even.

If $r$ is odd, the set $\Delta_{\text {odd }}$ of odd-indexed colors is distinguished and the quotient is a symmetric wonderful variety, so the surjectivity follows as in Proposition 4.3.

If $r$ is even, $H$ is reductive and the surjectivity follows as in Proposition 4.4.

5.2. Cotype $\mathrm{D}_{r}$. We test the triples of Lemma 4.7. $\left(D_{p}, D_{q}, F\right)$ where $p, q, r$ are odd, $p, q \leqslant r-2$ and either

$$
\begin{aligned}
& \text { - } p+q \leqslant r-1 \text { with } F=D_{p+q-2} \text { or } \\
& \text { - } p+q=r+1 \text { with } F=D_{r-1}+D_{r} \text {. }
\end{aligned}
$$

Let $m>1$ and set $r=2 m+1$. Let $V=\mathbb{C}^{m}$, and set $W=V \oplus \mathbb{C} e \oplus V^{*} \oplus \mathbb{C} \varepsilon$. On $W$ is defined a quadratic form such that $V$ and $V^{*}$ are orthogonal to $e$ and $\varepsilon$, moreover, $(e, \varepsilon)=1$ and $(e, e)=(\varepsilon, \varepsilon)=0$. Let $G=\operatorname{SL}(V) \times \operatorname{Spin}(W)$. We have $\mathfrak{h}_{0}=\mathfrak{s l}(V) \oplus \Lambda^{2} V \oplus V$, which is embedded as a subalgebra of $\mathfrak{g}$ as follows. First notice that we have a natural immersion of $\operatorname{SL}(V)$ in $\operatorname{Spin}(W)$ so that $\mathfrak{s l}(V)$ can be included diagonally in $\mathfrak{g}$. The action of $\Lambda^{2} V$ on $W$ is zero on $V, e$ and $\varepsilon$, while its action on $V^{*}$ is given by the identification of $\Lambda^{2} V$ with the antisymmetric maps from $V^{*}$ to $V$. The action of $V$ on $W$ is given as follows: if $v, u \in V$ and $\varphi \in V^{*}$ then

$$
v \cdot u=0 \quad v \cdot e=v \quad v \cdot \varphi=-\varphi(v)(e+\varepsilon) \quad v \cdot \varepsilon=v .
$$

Now let $\tilde{D}_{i}=D_{i}$ for $i$ odd or for $i \neq 2 m$ while $\tilde{D}_{2 m}=D_{2 m}+D_{2 m+1}$. We have

$$
V_{\tilde{D}_{2 i}}^{*}=\Lambda^{i} V^{*} \otimes \Lambda^{i} W \quad V_{\tilde{D}_{2 i+1}}^{*}=\Lambda^{i} V^{*} \otimes \Lambda^{i+1} W .
$$

The $\mathfrak{h}_{0}$-invariants in $V_{\tilde{D}_{2 i}}^{*}$ are generated by the vector $h_{2 i}$ corresponding to the identity element in $\Lambda^{i} V^{*} \otimes \Lambda^{i} V \subset \wedge^{i} V^{*} \otimes \wedge^{i} W$. Similarly, the $\mathfrak{h}_{0}$-invariants in 
$V_{\tilde{D}_{2 i+1}}^{*}$ are generated by the vector $h_{2 i+1}=h_{2 i} \wedge(e-\varepsilon)$. So that we have $h_{2 i}=\sum_{j_{1}<\ldots<j_{i}} \varphi_{j_{1} \ldots j_{i}} \otimes e_{j_{1} \ldots j_{i}} \quad$ and $\quad h_{2 i+1}=\sum_{j_{1}<\ldots<j_{i}} \varphi_{j_{1} \ldots j_{i}} \otimes\left(e_{j_{1} \ldots j_{i}} \wedge(e-\varepsilon)\right)$.

Finally, if $p=2 t+1$ and $q=2 s+1$ then the projection $\Phi: V_{\tilde{D}_{p}}^{*} \otimes V_{\tilde{D}_{q}}^{*} \longrightarrow V_{\tilde{D}_{p+q-2}}^{*}$ is given by

$$
\Phi((x \otimes w) \otimes(y \otimes z))=x \wedge y \otimes \tilde{\kappa}_{W}^{t+1 s+1}(w \otimes z)
$$

for $x \in \Lambda^{t} V^{*}, y \in \Lambda^{s} V^{*}, w \in \Lambda^{t+1} W$ and $z \in \Lambda^{s+1} W$. A direct computation shows that

$$
\Phi\left(h_{p} \otimes h_{q}\right)=(-1)^{t+s+1} 2\left(\begin{array}{c}
t+s \\
t
\end{array}\right) h_{p+q-2}
$$

5.3. Cotype $\mathrm{E}_{6}$. Let $V=\mathbb{C}^{3}$ and $W=V \oplus V^{*}$. Let $\mathfrak{g}=\mathfrak{s l}(W)$ and $\mathfrak{h}_{0}=$ $\mathfrak{s l}(V) \oplus \mathrm{S}^{2} V$ where the action of $\mathfrak{s l}(V)$ is the natural one and the action of $\mathrm{S}^{2} V$ is given by $b \cdot(v, \varphi)=(b(\varphi), 0)$ while the action on $W^{*}=V^{*} \oplus V$ is given by $b \cdot(\varphi, v)=(0,-b(\varphi))$.

We analyze the triples of Lemma 4.9. $\left(D_{1}, D_{3}, D_{2}\right),\left(D_{1}, D_{5}, D_{3}\right),\left(D_{1}, D_{6}, 0\right)$, $\left(D_{3}, D_{6}, D_{5}\right),\left(D_{5}, D_{6}, D_{2}\right)$.

The set of colors $\left\{D_{2}, D_{3}, D_{4}, D_{5}\right\}$ is distinguished and the associated quotient is a symmetric wonderful variety, so the third triple follows as in Proposition 4.3 . Therefore, by symmetry it is enough to analyze the last two triples. We need to compute $h_{3}, h_{5}, h_{6}$.

We have $V_{D_{6}}^{*}=\Lambda^{2} W$. Looking at the action of $\mathfrak{s l}(V)$ we find only one invariant in $V \otimes V^{*} \subset \wedge^{2} W$ corresponding to the identity in $\operatorname{End}\left(V^{*}\right)$. Hence we get $h_{6}=$ $e_{1} \wedge \varphi_{1}+e_{2} \wedge \varphi_{2}+e_{3} \wedge \varphi_{3}$.

The representation $V_{D_{5}}^{*}$ is contained in $\Lambda^{2} W \otimes W^{*}$ and it is the kernel of $\kappa_{W}$. It contains two invariants under the action of $\mathfrak{s l}(V): x \in \Lambda^{2} V \otimes V \simeq \operatorname{End}(V)$ and $y \in \Lambda^{2} V^{*} \otimes V^{*} \simeq \operatorname{End}\left(V^{*}\right)$. Notice that $\Lambda^{2} W \otimes W^{*} \simeq V_{D_{5}}^{*} \oplus W$ so both $x, y \in V_{D_{5}}^{*}$. Moreover, the action of $\mathrm{S}^{2} V$ on $x$ is clearly trivial while it is not on $y$. So we have $h_{5}=e_{12} \otimes \varphi_{3}^{*}-e_{13} \otimes \varphi_{2}^{*}+e_{23} \otimes \varphi_{1}^{*}$.

Similarly, we have $V_{D_{3}}^{*}=\operatorname{ker} \kappa_{W^{*}} \subset \Lambda^{2} W^{*} \otimes W$ and $h_{3}=\varphi_{12}^{*} \otimes e_{3}-\varphi_{13}^{*} \otimes e_{2}+$ $\varphi_{23}^{*} \otimes e_{1}$.

5.3.1. Analysis of the triple $\left(D_{5}, D_{6}, D_{2}\right)$. We have $V_{D_{2}}^{*}=\Lambda^{3} W$. Consider the map $\Phi: \Lambda^{2} W \otimes \Lambda^{2} W \otimes W^{*} \longrightarrow \Lambda^{3} W$ given by

$$
\Phi(x \otimes y \otimes \varphi)=\kappa_{W}(x \otimes \varphi) \wedge y
$$

for $x, y \in \Lambda^{2} W$ and $\varphi \in W^{*}$. A direct computation shows that $\Phi\left(h_{6} \otimes h_{5}\right)=$ $3 e_{123} \neq 0$.

5.3.2. Analysis of the triple $\left(D_{3}, D_{6}, D_{5}\right)$. Consider the map $\Phi: \Lambda^{2} W \otimes \Lambda^{2} W^{*} \otimes$ $W \longrightarrow \Lambda^{2} W \otimes W^{*}$ given by

$$
\Phi((x \wedge y) \otimes(\varphi \wedge \psi) \otimes w)=\left(\kappa_{W}(x \wedge y \otimes \varphi) \wedge w\right) \otimes \psi-\left(\kappa_{W}(x \wedge y \otimes \psi) \wedge w\right) \otimes \varphi
$$

for $x, y, w \in W$ and $\varphi, \psi \in W^{*}$. A direct computation shows that $\Phi\left(h_{6} \otimes h_{3}\right)=2 h_{5}$. 
5.4. Cotype $\mathrm{E}_{7}$. Let $V=\mathbb{C}^{3}$ and set $W=V \oplus V^{*} \oplus \mathbb{C} e, \mathfrak{h}_{0}=\mathfrak{s l}(V) \oplus \mathrm{S}^{2} V \oplus \Lambda^{2} V \oplus V$ and $\mathfrak{g}=\mathfrak{s l}(W)$. Recall that we identify $\mathrm{S}^{2} V \oplus \Lambda^{2} V$ with the decomposition of $\operatorname{Hom}\left(V^{*}, V\right)$ into symmetric and antisymmetric matrices. We have an action of $\mathfrak{h}_{0}$ on $W$ given as follows. Let $(a, b, \omega, u) \in \mathfrak{h}_{0}$ and $(v, \varphi, \lambda e) \in W$, then

$$
\begin{aligned}
& a \cdot v=a(v) \quad b \cdot v=0 \quad \omega \cdot v=0 \quad u \cdot v=0 \\
& a \cdot \varphi=-a^{t}(\varphi) \quad b \cdot \varphi=b(\varphi) \quad \omega \cdot \varphi=\omega(\varphi) \quad u \cdot \varphi=0 \\
& a \cdot e=0 \quad b \cdot e=0 \quad \omega \cdot e=\gamma(\omega) \quad u \cdot e=u
\end{aligned}
$$

where $\gamma=\gamma_{2}: \Lambda^{2} V \longrightarrow V^{*}$ is defined as above. This defines an immersion of $\mathfrak{h}_{0}$ into $\mathfrak{s l}(W)$ whose image is closed under the Lie bracket. Indeed, if $(a, b, \omega, u) \in \mathfrak{h}_{0}$ and $\left(a^{\prime}, b^{\prime}, \omega^{\prime}, u^{\prime}\right) \in \mathfrak{h}_{0}$, then

$$
\begin{aligned}
& {\left[a, a^{\prime}\right]=a a^{\prime}-a^{\prime} a \quad[a, b]=a b+b a^{t} \quad[a, \omega]=a \omega+\omega a^{t} \quad[a, u]=a(u)} \\
& {\left[b, b^{\prime}\right]=0 \quad[b, \omega]=b(\gamma(\omega)) \quad[b, u]=0 \quad\left[\omega, \omega^{\prime}\right]=0 \quad[\omega, u]=0 \quad\left[u, u^{\prime}\right]=0 .}
\end{aligned}
$$

It is also useful to write the action on $W^{*}$ which is given as follows. We have $W^{*}=V^{*} \oplus V \oplus \mathbb{C} e^{*}$, then

$$
\begin{aligned}
& a \cdot \varphi=-a^{t}(\varphi) \quad b \cdot \varphi=-b(\varphi) \quad \omega \cdot \varphi=\omega(\varphi) \quad u \cdot \varphi=-\varphi(u) e^{*} \\
& a \cdot v=a(v) \quad b \cdot v=0 \quad \omega \cdot v=-\langle\omega, v\rangle e^{*} \quad u \cdot v=0 \\
& a \cdot e^{*}=0 \quad b \cdot e^{*}=0 \quad \omega \cdot e^{*}=\gamma(\omega) \quad u \cdot e^{*}=u
\end{aligned}
$$

The triples of Lemma 4.9 are $\left(D_{1}, D_{6}, D_{3}\right)$ and $\left(D_{6}, D_{6}, D_{2}+D_{7}\right)$.

5.4.1. Computation of $h_{1}$. The representation associated to $D_{1}$ is $\Lambda^{4} W$. Looking at the action of $\mathfrak{s l}(V)$ we get three invariants: the vector $x \in \Lambda^{2} V \otimes \Lambda^{2} V^{*}$ corresponding to the identity in $\operatorname{End}\left(\Lambda^{2} V\right)$, the vector $y=e_{123} \wedge e \in \Lambda^{3} V \otimes \mathbb{C} e$ and the vector $z=\varphi_{123} \wedge e \in \Lambda^{3} V^{*} \otimes \mathbb{C} e$. Hence the invariant is a linear combination of these vectors. A small computation shows that

$$
h_{1}=2 y-x=2 e_{123} \wedge e-e_{12} \wedge \varphi_{12}-e_{13} \wedge \varphi_{13}-e_{23} \wedge \varphi_{23} .
$$

5.4.2. Computation of $h_{6}$. The representation associated to $D_{6}$ is the kernel of the map $\kappa_{W}$ in $\Lambda^{2} W \otimes W^{*}$. In $\Lambda^{2} W \otimes W^{*}$ there are five invariant vectors under $\mathfrak{s l}(V)$ : $x_{1} \in \Lambda^{2} V \otimes V, x_{2} \in \Lambda^{2} V^{*} \otimes V^{*}, y_{1} \in(V \wedge \mathbb{C} e) \otimes V^{*}, y_{2} \in\left(V^{*} \wedge \mathbb{C} e\right) \otimes V$ and $z \in\left(V \wedge V^{*}\right) \otimes \mathbb{C} e^{*}$. A small computation shows that

$$
\begin{aligned}
h_{6}=2 x_{1}+z= & 2 e_{12} \otimes \varphi_{3}^{*}-2 e_{13} \otimes \varphi_{2}^{*}+2 e_{23} \otimes \varphi_{1}^{*}+\left(e_{1} \wedge \varphi_{1}\right) \otimes e^{*} \\
& +\left(e_{2} \wedge \varphi_{2}\right) \otimes e^{*}+\left(e_{3} \wedge \varphi_{3}\right) \otimes e^{*} .
\end{aligned}
$$

5.4.3. Analysis of the triple $\left(D_{1}, D_{6}, D_{3}\right)$. The representation associated to the color $D_{3}$ is the kernel of the wedge product in $W \otimes \Lambda^{4} W \longrightarrow \Lambda^{5} W$. We consider the map $\Phi: \Lambda^{4} W \otimes \Lambda^{2} W \otimes W^{*} \longrightarrow W \otimes \Lambda^{4} W$ given by

$$
\Phi\left(u \otimes\left(v_{1} \wedge v_{2}\right) \otimes \varphi\right)=v_{1} \otimes\left(\kappa_{W}^{41}(u \otimes \varphi) \wedge v_{2}\right)-v_{2} \otimes\left(\kappa_{W}^{41}(u \otimes \varphi) \wedge v_{1}\right) .
$$

A direct computation shows that

$$
\Phi\left(h_{1} \otimes h_{6}\right)=-4\left(e_{1} \otimes\left(e_{123} \wedge \varphi_{1}\right)+e_{2} \otimes\left(e_{123} \wedge \varphi_{2}\right)+e_{3} \otimes\left(e_{123} \wedge \varphi_{3}\right)\right) \neq 0 .
$$


5.4.4. Analysis of the triple $\left(D_{6}, D_{6}, D_{2}+D_{7}\right)$. The representation $V_{D_{2}+D_{7}}^{*}$ associated to the color $D_{2}+D_{7}$ is the kernel of the map $\kappa_{W}^{31}: \Lambda^{3} W \otimes W^{*} \longrightarrow \Lambda^{2} W$. We consider the map $\Psi:\left(\wedge^{2} W \otimes W^{*}\right) \otimes\left(\Lambda^{2} W \otimes W^{*}\right) \longrightarrow \Lambda^{3} W \otimes W^{*}$ given by

$$
\Psi((u \otimes \varphi) \otimes(v \otimes \psi))=\left(u \wedge \kappa_{W}(v \otimes \varphi)\right) \otimes \psi .
$$

A direct computation shows that

$$
\Psi\left(h_{6} \otimes h_{6}\right)=-6 e_{123} \otimes e^{*} \in V_{D_{2}+D_{7}}^{*} .
$$

5.5. Cotype $\mathrm{E}_{8}$. Let $V=\mathbb{C}^{4}$ and set $W=V \oplus \Lambda^{2} V \oplus V^{*}=X \oplus Y \oplus Z$. On $W$ it is defined a non-degenerate symmetric bilinear form such that $V \oplus V^{*}$ is orthogonal to $\Lambda^{2} V$ and such that restricted to $V \oplus V^{*}$ and to $\Lambda^{2} V$ is the one introduced at the beginning of this section.

Let $A$ be the kernel of the wedge product $V \otimes \Lambda^{2} V \longrightarrow \Lambda^{3} V$ and set $\mathfrak{h}_{0}=$ $\mathfrak{s l}(V) \oplus A \oplus \Lambda^{2} V$. There is an action of $\mathfrak{h}_{0}$ on $W$ given as follows. Let $(a, b, \alpha) \in \mathfrak{h}_{0}$ and $(v, \omega, \varphi) \in W$ then

$$
\begin{array}{lll}
a \cdot v=a(v) & b \cdot v=0 & \alpha \cdot v=0 \\
a \cdot \omega=a(\omega) & b \cdot \omega=\gamma(b \otimes \omega) & \alpha \cdot \omega=0 \\
a \cdot \varphi=-a^{t}(\varphi) & b \cdot \varphi=\delta(b \otimes \varphi) & \alpha \cdot \varphi=\kappa_{V}(\alpha \otimes \varphi)
\end{array}
$$

where $\gamma, \delta$ are defined as follows:

$$
\begin{array}{ll}
\gamma: V \otimes \Lambda^{2} V \otimes \Lambda^{2} V \longrightarrow V & \gamma\left(v \otimes \alpha \otimes \alpha^{\prime}\right)=\left(\alpha, \alpha^{\prime}\right) v \\
\delta: V \otimes \Lambda^{2} V \otimes V^{*} \longrightarrow \Lambda^{2} V & \delta(v \otimes \alpha \otimes \varphi)=\varphi(v) \alpha
\end{array}
$$

The action of $\mathfrak{h}_{0}$ on $W$ defines an immersion of $\mathfrak{h}_{0}$ into $\mathfrak{s o}(W)$, whose image is closed under the Lie bracket and more explicitly for $a, a^{\prime} \in \mathfrak{s l}(V), b, b^{\prime} \in A$ and $\alpha, \alpha^{\prime} \in \Lambda^{2} V$ we have

$$
\begin{aligned}
& {\left[a, a^{\prime}\right]=a a^{\prime}-a^{\prime} a} \\
& {[a, b]=a(b)} \\
& {[a, \alpha]=a(\alpha)} \\
& {\left[b, b^{\prime}\right]=-\zeta\left(b \otimes b^{\prime}\right)} \\
& {[b, \alpha]=0} \\
& {\left[\alpha, \alpha^{\prime}\right]=0}
\end{aligned}
$$

where $\zeta$ is the map

$$
\zeta:\left(V \otimes \Lambda^{2} V\right) \otimes\left(V \otimes \Lambda^{2} V\right) \longrightarrow \Lambda^{2} V \quad \zeta\left((u \otimes \alpha) \otimes\left(u^{\prime} \otimes \alpha^{\prime}\right)\right)=\left(\alpha, \alpha^{\prime}\right) u \wedge u^{\prime} .
$$

The triples of Lemma 4.9 follow: $\left(D_{1}, D_{1}, D_{2}\right),\left(D_{1}, D_{5}, 2 D_{2}\right),\left(D_{1}, D_{7}, D_{3}\right)$, $\left(D_{1}, D_{8}, D_{7}\right),\left(D_{3}, D_{8}, D_{5}\right),\left(D_{5}, D_{8}, D_{2}+D_{7}\right)$ and $\left(D_{7}, D_{8}, D_{2}\right)$.

5.5.1. Computation of $h_{1}$. The representation associated to $D_{1}$ is $\Lambda^{3} W$. Looking at the action of $\mathfrak{s l}(V)$ we get two invariants: $x \in \Lambda^{2} X \otimes Y \simeq \Lambda^{2} V \otimes \Lambda^{2} V$ and $y \in Y \otimes \Lambda^{2} Z \simeq \Lambda^{2} V \otimes \Lambda^{2} V^{*}$. We notice now that $X$ is invariant also by the action of $A$ and $\Lambda^{2} V$. Indeed, if $b \in A$, then $b \cdot x \in \Lambda^{3} X \simeq \Lambda^{3} V$; in particular, we get a $\mathfrak{s l}(V)$-equivariant map from $A$ to $\Lambda^{3} V$, which must be zero. A similar argument proves that $\alpha \cdot x=0$ for $\alpha \in \Lambda^{2} V$. Finally, it is easy to check that if $b=e_{1} \wedge e_{12}$, which belongs to $A$, then $b \cdot y \neq 0$. Hence

$h_{1}=x=e_{1} \wedge e_{2} \wedge e_{34}-e_{1} \wedge e_{3} \wedge e_{24}+e_{1} \wedge e_{4} \wedge e_{23}+e_{2} \wedge e_{3} \wedge e_{14}-e_{2} \wedge e_{4} \wedge e_{13}+e_{3} \wedge e_{4} \wedge e_{12}$. 
5.5.2. Computation of $h_{3}, h_{5}, h_{7}$. Let $P$ be the parabolic of $\operatorname{Spin}(W)$ defined by $g(V) \subset V$. Notice that $H \subset P$. Let $U$ be the unipotent radical of $P, L$ its Levi subgroup, and $L^{\mathrm{ss}}$ its semisimple part. Notice that $L^{\mathrm{ss}} \simeq \mathrm{SL}(4) \times \mathrm{SL}(4)$. Let $T \subset G$ be the maximal torus of elements acting diagonally on $W$ with respect to the basis $e_{1}, \ldots, e_{4}, e_{12}, e_{13}, e_{14}, e_{23},-e_{24}, e_{34}, \varphi_{4}, \ldots, \varphi_{1}$ and $B \subset G$ the subgroup of elements whose action on $W$ is upper triangular with respect to this basis. The natural action of $\mathrm{SL}(V)$ on $W$ induces an embedding $\mathrm{SL}(V) \longrightarrow L$, that on diagonal elements takes the form

$$
\left(t_{1}, t_{2}, t_{3}, t_{4}\right) \longmapsto\left(t_{1}, t_{2}, t_{3}, t_{4}, t_{1} t_{2}, t_{1} t_{3}, t_{1} t_{4}, t_{2} t_{3}, t_{2} t_{4}, t_{3} t_{4}, t_{4}^{-1}, t_{3}^{-1}, t_{2}^{-1}, t_{1}^{-1}\right) .
$$

The set of colors $\left\{D_{1}, D_{4}, D_{6}, D_{8}\right\}$ is distinguished, and $K_{0}=H_{0} U$ is the intersection of the kernels of the multiplicative characters of the stabilizer $K$ of a point in the open orbit of the quotient. Therefore, $h_{3}, h_{5}, h_{7}$ must be $K_{0}$-invariant vectors in $V_{D_{i}}^{*}$. Let $W_{i}=\left(V_{D_{i}}^{*}\right)^{U}$. This is an irreducible representation of $L$ of the same highest weight as $V_{D_{i}}^{*}: \omega_{3}+\omega_{7}$ for $i=3, \omega_{2}+\omega_{5}$ for $i=5$, and $\omega_{1}+\omega_{6}$ for $i=7$. When we restrict these representations to $\mathrm{SL}(V)$ we get

$$
W_{3} \simeq \Lambda^{3} V \otimes V \quad W_{5} \simeq \Lambda^{2} V \otimes \Lambda^{2} V \quad W_{7} \simeq V \otimes \Lambda^{3} V ;
$$

in particular, there is an invariant element under $\mathfrak{h}_{0}$.

For notational convenience, here and below, set

$$
e_{5}=e_{12}, e_{6}=e_{13}, e_{7}=e_{14}, \varphi_{7}=e_{23}, \varphi_{6}=-e_{24}, \varphi_{5}=e_{34} .
$$

Let $U \subset W$ be the subspace spanned by $e_{1}, \ldots, e_{7}$, so that $W$ becomes $U \oplus U^{*}$.

Now we need to describe the spin representations. Consider the whole exterior algebra $\Lambda U^{*}$. It decomposes into odd and even degree parts $\Lambda^{\text {odd }} U^{*} \oplus \Lambda^{\text {even }} U^{*}$. Since the $G$-action we are going to define is not the natural one, we stress the difference by using a different notation: set $\psi_{i_{1} \ldots i_{k}}=\varphi_{i_{1} \ldots i_{k}}$. Define the map $\sigma: W \otimes \Lambda U^{*} \longrightarrow \Lambda U^{*}$ such that

$$
\begin{gathered}
\sigma\left(e_{i} \otimes \psi_{i_{1} \ldots i_{k}}\right)=\kappa_{U^{*}}^{k 1}\left(\varphi_{i_{1} \ldots i_{k}} \otimes e_{i}\right), \\
\sigma\left(\varphi_{i} \otimes \psi_{i_{1} \ldots i_{k}}\right)=\varphi_{i} \wedge \varphi_{i_{1} \ldots i_{k}},
\end{gathered}
$$

and more generally the map $\sigma^{n}: \otimes^{n} W \otimes \Lambda U^{*} \longrightarrow \Lambda U^{*}$ such that

$$
\sigma^{n}\left(w_{1} \otimes \cdots \otimes w_{n} \otimes y\right)=\sigma\left(w_{1} \otimes \sigma\left(\cdots \otimes \sigma\left(w_{n} \otimes y\right) \ldots\right)\right)
$$

which we can restrict to $\Lambda^{n} W$ if we think of $w_{1} \wedge \cdots \wedge w_{n}$ as the corresponding antisymmetric tensor, with coefficient $\frac{1}{n !}$.

To get the spin representations we can just take the map $\sigma^{2}$; indeed, notice that $\Lambda^{2} W$ identifies with $\mathfrak{s o}(W)$ through $w_{1} \wedge w_{2}=\left(w_{2},\right) w_{1}-\left(w_{1},\right) w_{2}$. We thus have that the vector $\psi_{i_{1} \ldots i_{k}}$ has weight

$$
\frac{1}{2}\left(\sum_{i \notin\left\{i_{1}, \ldots, i_{k}\right\}} \varepsilon_{i}-\sum_{i \in\left\{i_{1}, \ldots, i_{k}\right\}} \varepsilon_{i}\right),
$$

$V\left(\omega_{6}\right) \simeq \Lambda^{\text {odd }} U^{*}$ and $V\left(\omega_{7}\right) \simeq \Lambda^{\text {even }} U^{*}$ 
We get the following expressions of the $H_{0}$-invariants:

$$
\begin{aligned}
h_{3}= & e_{123} \otimes \psi_{65}+e_{124} \otimes \psi_{75}+e_{134} \otimes \psi_{76}-e_{234} \otimes \psi_{\emptyset}, \\
h_{5}= & e_{12} \otimes e_{1234} \wedge \varphi_{5}+e_{13} \otimes e_{1234} \wedge \varphi_{6}+e_{14} \otimes e_{1234} \wedge \varphi_{7}+e_{23} \otimes e_{12347} \\
& -e_{24} \otimes e_{12346}+e_{34} \otimes e_{12345}, \\
h_{7}= & e_{1} \otimes \psi_{765}-e_{2} \otimes \psi_{5}-e_{3} \otimes \psi_{6}-e_{4} \otimes \psi_{7} .
\end{aligned}
$$

5.5.3. Computation of $h_{8}$. The representation $V_{D_{8}}^{*}$ is the spin representation of highest weight $\omega_{6}$. By direct computation one can show that the only $H_{0}$-invariant is given by

$$
h_{8}=\psi_{1}+\psi_{762}-\psi_{753}+\psi_{654}
$$

5.5.4. Analysis of the triple $\left(D_{1}, D_{1}, D_{2}\right)$. The representation associated with $D_{2}$ is $\Lambda^{4} \mathrm{~W}$. The $H_{0}$-invariant $h_{2}$ is $e_{1234}$. Indeed, the set of colors $\Delta \backslash\left\{D_{2}\right\}$ is distinguished and the quotient is homogeneous, hence an $H$-semi-invariant in $V_{D_{2}}^{*}$ must be $P$-semiinvariant.

Here we get

$$
\tilde{\kappa}_{W}^{33}\left(h_{1} \otimes h_{1}\right)=3 h_{2}
$$

5.5.5. Analysis of the triple $\left(D_{1}, D_{5}, 2 D_{2}\right)$. Consider the map $\Phi: \Lambda^{3} W \otimes \Lambda^{2} W \otimes$ $\Lambda^{5} W \longrightarrow \Lambda^{4} W \otimes \Lambda^{4} W$ such that

$$
\begin{aligned}
& \Phi\left(\left(w_{1} \wedge w_{2} \wedge w_{3}\right) \otimes x \otimes y\right)=\left(w_{1} \wedge w_{2} \wedge x\right) \otimes \kappa_{W}^{51}\left(y \otimes w_{3}\right) \\
& -\left(w_{1} \wedge w_{3} \wedge x\right) \otimes \kappa_{W}^{51}\left(y \otimes w_{2}\right)+\left(w_{2} \wedge w_{3} \wedge x\right) \otimes \kappa_{W}^{51}\left(y \otimes w_{1}\right) .
\end{aligned}
$$

We have

$$
\Phi\left(h_{1} \otimes h_{5}\right)=6 h_{2} \otimes h_{2}
$$

5.5.6. Analysis of the triple $\left(D_{1}, D_{7}, D_{3}\right)$. Consider the map

$$
\Phi: \Lambda^{3} W \otimes W \otimes \Lambda^{\text {odd }} U^{*} \longrightarrow \Lambda^{3} W \otimes \Lambda^{\text {even }} U^{*}
$$

such that

$$
\begin{aligned}
& \Phi\left(\left(w_{1} \wedge w_{2} \wedge w_{3}\right) \otimes w \otimes \psi\right)=\left(w_{1} \wedge w_{2} \wedge w\right) \otimes \sigma\left(w_{3} \otimes \psi\right) \\
& \quad-\left(w_{1} \wedge w_{3} \wedge w\right) \otimes \sigma\left(w_{2} \otimes \psi\right)+\left(w_{2} \wedge w_{3} \wedge w\right) \otimes \sigma\left(w_{1} \otimes \psi\right) .
\end{aligned}
$$

We get

$$
\Phi\left(h_{1} \otimes h_{7}\right)=3 h_{3}
$$

5.5.7. Analysis of the triple $\left(D_{1}, D_{8}, D_{7}\right)$. Consider the map $\Phi: \Lambda^{3} W \otimes \Lambda^{\text {odd }} U^{*} \longrightarrow$ $W \otimes \Lambda^{\text {odd }} U^{*}$ such that

$$
\begin{aligned}
& \Phi\left(\left(w_{1} \wedge w_{2} \wedge w_{3}\right) \otimes \psi\right)=w_{1} \otimes \sigma^{2}\left(\left(w_{2} \wedge w_{3}\right) \otimes \psi\right) \\
& \quad-w_{2} \otimes \sigma^{2}\left(\left(w_{1} \wedge w_{3}\right) \otimes \psi\right)+w_{3} \otimes \sigma^{2}\left(\left(w_{1} \wedge w_{2}\right) \otimes \psi\right) .
\end{aligned}
$$

We get

$$
\Phi\left(h_{1} \otimes h_{8}\right)=-3 h_{7}
$$


5.5.8. Analysis of the triple $\left(D_{3}, D_{8}, D_{5}\right)$. On $\Lambda^{6} W$ there is a symmetric bilinear form $($,$) naturally induced by the given form on W$. On the other hand, $V\left(\omega_{6}\right)$ and $V\left(\omega_{7}\right)$ are dual to each other, so there is a natural non-degenerate pairing $\langle$,$\rangle .$ Consider the map $\Psi: \Lambda^{\text {odd }} U^{*} \otimes \Lambda^{\text {even }} U^{*} \longrightarrow \Lambda^{6} W$ such that

$$
\left(u, \Psi\left(\psi \otimes \psi^{\prime}\right)\right)=\left\langle\sigma^{6}(u) \psi, \psi^{\prime}\right\rangle
$$

and the map $\Phi: \Lambda^{3} W \otimes \Lambda^{\text {odd }} U^{*} \otimes \Lambda^{\text {even }} U^{*} \longrightarrow \Lambda^{2} W \otimes \Lambda^{5} W$ such that

$$
\begin{aligned}
\Phi\left(\left(w_{1} \wedge w_{2} \wedge w_{3}\right) \otimes \psi \otimes \psi^{\prime}\right) \\
=\left(w_{1} \wedge w_{2}\right) \otimes \tilde{\kappa}_{W}^{21}\left(\Psi\left(\psi \otimes \psi^{\prime}\right) \otimes w_{3}\right) \\
\quad-\left(w_{1} \wedge w_{3}\right) \otimes \tilde{\kappa}_{W}^{21}\left(\Psi\left(\psi \otimes \psi^{\prime}\right) \otimes w_{2}\right) \\
\quad+\left(w_{2} \wedge w_{3}\right) \otimes \tilde{\kappa}_{W}^{21}\left(\Psi\left(\psi \otimes \psi^{\prime}\right) \otimes w_{1}\right) .
\end{aligned}
$$

We get

$$
\Phi\left(h_{3} \otimes h_{8}\right)=h_{5} .
$$

5.5.9. Analysis of the triple $\left(D_{5}, D_{8}, D_{2}+D_{7}\right)$. Consider the map $\Phi: \Lambda^{2} W \otimes \Lambda^{5} W \otimes$ $\Lambda^{\text {odd }} U^{*} \longrightarrow \Lambda^{4} W \otimes W \otimes \Lambda^{\text {odd }} U^{*}$ such that

$$
\begin{aligned}
\Phi\left(\left(w_{1}\right.\right. & \left.\left.\wedge w_{2}\right) \otimes\left(z_{1} \wedge \cdots \wedge z_{5}\right) \otimes \psi\right) \\
= & \sum_{i}(-1)^{i+1}\left(z_{1} \wedge \cdots \wedge \hat{z}_{i} \wedge \cdots \wedge z_{5}\right) \otimes w_{2} \otimes \sigma^{2}\left(\left(z_{i} \wedge w_{1}\right) \otimes \psi\right) \\
& \quad-\sum_{i}(-1)^{i+1}\left(z_{1} \wedge \cdots \wedge \hat{z}_{i} \wedge \cdots \wedge z_{5}\right) \otimes w_{1} \otimes \sigma^{2}\left(\left(z_{i} \wedge w_{2}\right) \otimes \psi\right) .
\end{aligned}
$$

We get

$$
\Phi\left(h_{5} \otimes h_{8}\right)=-3 h_{2} \otimes h_{7} .
$$

5.5.10. Analysis of the triple $\left(D_{7}, D_{8}, D_{2}\right)$. Consider the map $\Phi: W \otimes \Lambda^{\text {odd }} U^{*} \otimes$ $\Lambda^{\text {odd }} U^{*} \longrightarrow \Lambda^{\text {odd }} U^{*} \otimes \Lambda^{\text {even }} U^{*}$ such that

$$
\Phi\left(w \otimes \psi \otimes \psi^{\prime}\right)=\sigma\left(w \otimes \psi^{\prime}\right) \otimes \psi .
$$

Here we get that $\Phi\left(h_{7} \otimes h_{8}\right)$ is a $U$-invariant vector of weight $\omega_{4}$.

\subsection{Projective normality of comodel wonderful varieties.}

Proof of Theorem 5.2. As in the case of the model wonderful varieties, by Lemma 2.3 , we are reduced to study the low fundamental triples.

Recall that in the model case we have classified, for every $G$ of connected Dynkin type, the low fundamental triples $(D, E, F)$ of the model wonderful $G$-variety (of simply connected type) such that $\operatorname{supp}_{S}(D+E-F)=S$.

In the comodel case, these correspond to the low fundamental triples $(D, E, F)$ of the comodel wonderful varieties of connected Dynkin cotype such that $\operatorname{cosupp}(D+$ $E-F)=\Delta$, where

$$
\operatorname{cosupp} \gamma=\left\{D \in \Delta:\left\langle\alpha^{\vee}, \omega_{D}\right\rangle \neq 0 \text { for some } \alpha \in \operatorname{supp}_{S} \gamma\right\} \text {. }
$$

Let us go back to the model case. Let $S$ be the set of simple roots. Let $M$ be a model wonderful variety with set of colors $\Delta$. Recall that for every low fundamental triple $(D, E, F) \operatorname{supp}_{S}(D+E-F)=S^{\prime}$ is connected. Hence $(D, E, F)$ corresponds to a low fundamental triple $(D, E, \widetilde{F})$, of a model wonderful variety $N$ (with set of colors $\widetilde{\Delta} \subset \Delta$ ) of connected Dynkin type (with $S^{\prime}$ as set of simple roots) such 
that $M$ is parabolic induction of $N$, with $D, E \in \widetilde{\Delta}, D+E-F=D+E-\widetilde{F}$ and $\operatorname{supp}_{S^{\prime}}(D+E-\widetilde{F})=S^{\prime}$.

This can be translated into the comodel case. Every low fundamental triple $(D, E, F)$ of a comodel wonderful variety $M$ (with set of colors $\Delta$ ) corresponds to a low fundamental triple $(D, E, \widetilde{F})$ of a comodel wonderful variety $N$ of connected Dynkin cotype (with set of colors $\widetilde{\Delta} \subset \Delta$ ) such that $M$ is parabolic induction of $N$, with $D, E \in \widetilde{\Delta}, D+E-F=D+E-\widetilde{F}$ and $\operatorname{cosupp}(D+E-\widetilde{F})=\widetilde{\Delta}$.

Since we have already checked the surjectivity for all such low fundamental triples, we can conclude by applying Proposition 1.6 as in the proof of Theorem 4.1 (see Section 4.8).

\section{ON THE NORMALITY OF SPHERICAL ORBIT CLOSURES IN SIMPLE PROJECTIVE SPACES}

Let $M$ be a wonderful $G$-variety with set of spherical roots $\Sigma$ and set of colors $\Delta$. Denote by $H$ the stabilizer of a point $x_{0}$ in the open $G$-orbit.

If $D$ is in $\mathbb{N} \Delta$ and $h_{D} \in\left(V_{D}^{*}\right)_{\xi_{D}}^{(H)}$ is the associated $H$-eigenvector, consider the orbit closure

$$
X_{D}=\overline{G \cdot\left[h_{D}\right]} \subset \mathbb{P}\left(V_{D}^{*}\right),
$$

which is a simple possibly non-normal spherical variety (recall that a spherical variety is called simple if it contains a unique closed orbit). We have a natural morphism $\phi_{D}: M \longrightarrow X_{D}$ such that $\phi_{D}^{*} \mathcal{O}(1)=\mathcal{L}_{D}$.

By [29, Corollary 7.6] and [8, Corollary 2.4.2.2], every spherical orbit in a simple projective space always admits a wonderful compactification $M$, so it is of the shape $G \cdot\left[h_{D}\right]$ for some $D \in \mathbb{N} \Delta$. As a consequence of the results of the previous sections, here we show that under some special assumptions on $M$ the variety $X_{D}$ is always normal.

The variety $X_{D}$ was studied by G. Pezzini in [39] when $D$ is ample, that is, $D \in \mathbb{N}_{>0} \Delta$. Under this assumption, either $X_{D}$ is isomorphic to $M$ or it is not even normal. In case $X_{D} \simeq M$, then $M$ is called strict; this is equivalent to the conditions $H=N_{G}(H)$ and $\Sigma \cap S=\varnothing$. There are essentially two main classes of examples of strict wonderful varieties: the adjoint symmetric wonderful varieties and the model wonderful varieties.

When $D$ is not ample, the variety $X_{D}$ was then studied in [37] in the symmetric case and in 22] in general. More precisely, the orbit structure of $X_{D}$ and that of its normalization $\widetilde{X}_{D}$ were analyzed. In particular, it was proved that the normalization $\widetilde{X}_{D} \longrightarrow X_{D}$ is always bijective if $M$ is adjoint symmetric or if $G$ is of simply laced type and $M$ is strict, while the main counterexamples where bijectivity fails, in the strict case, arise with the model wonderful varieties for groups of not simply laced type.

We now consider a special class of big divisors on $M$. We say that $D \in \mathbb{N} \Delta$ is a faithful divisor on $M$ if $\phi_{D}$ restricts to an open embedding of $G / H$ in $\mathbb{P}\left(V_{D}^{*}\right)$, i.e., if $H$ equals the stabilizer of $\left[h_{D}\right]$. The formalism of distinguished sets of colors allows us to characterize combinatorially the faithful divisors. For simplicity, we restrict ourselves to the case of a strict wonderful variety.

Proposition 6.1 (see [ㅁ, Proposition 2.4.3]). Let $M$ be a strict wonderful variety and let $D \in \mathbb{N} \Delta$. Then $D$ is faithful if and only if every distinguished subset of $\Delta$ intersects $\operatorname{supp}(D)$. 
Let $D \in \mathbb{N} \Delta$, denote $\widetilde{A}(D)=\bigoplus_{n \in \mathbb{N}} \Gamma\left(M, \mathcal{L}_{n D}\right)$ and denote $A(D) \subset \widetilde{A}(D)$ the subalgebra generated by $V_{D} \subset \Gamma\left(M, \mathcal{L}_{D}\right)$. Let $\widetilde{X}_{D}$ be the image of $M$ in $\mathbb{P}\left(\Gamma\left(M, \mathcal{L}_{D}\right)^{*}\right)$ via the morphism $\widetilde{\phi}_{D}$ associated to the complete linear system of $D$. Then $\widetilde{A}(D)$ is identified with the projective coordinate ring of $\widetilde{X}_{D}$, whereas $A(D)$ is identified with the projective coordinate ring of $X_{D}$. Notice that we have a natural projection $\beta_{D}: \widetilde{X}_{D} \longrightarrow X_{D}$ such that $\phi_{D}=\widetilde{\phi}_{D} \circ \beta_{D}$. Since $M$ is smooth, $\widetilde{A}(D)$ is an integrally closed algebra, therefore $\widetilde{X}_{D}$ is normal. Moreover, we have the following.

Proposition 6.2 (see [13, Proposition 2.1]). The algebra $\widetilde{A}(D)$ is integral over $A(D)$.

It follows that $\beta_{D}: \widetilde{X}_{D} \longrightarrow X_{D}$ is the normalization if and only if it is birational. Clearly this is the case if $D$ is minuscule or faithful. On the other hand, $\beta_{D}$ is not necessarily birational: if $M$ is the model wonderful variety of $\mathrm{C}_{4}$, then $A\left(D_{2}\right) \subset$ $\widetilde{A}\left(D_{2}\right)$ do not have the same quotient field. However, we have the following.

Proposition 6.3. Suppose that $M$ is adjoint symmetric or that $G$ is simply laced and $M$ is strict. Then $\widetilde{A}(D)$ and $A(D)$ have the same quotient field.

Proof. Let $\Delta_{D} \subset \Delta$ be the maximal distinguished subset which does not intersect $\operatorname{supp}(D)$, and denote by $M^{\prime}$ the quotient of $M$ by $\Delta_{D}$ and by $M^{\prime \prime}$ the wonderful compactification of $G \cdot\left[h_{D}\right]$. By [22, Corollary 3.7] and [22, Remark 3.8], $M^{\prime}$ and $M^{\prime \prime}$ have the same dimension, and we have $M^{\prime} \neq M^{\prime \prime}$ if and only if some spherical root of $M^{\prime \prime}$ is the double of a spherical root of $M^{\prime}$.

On the other hand, by the quotient construction, the spherical roots of $M^{\prime}$ are contained in $\mathbb{N} \Sigma$. By the description of the possible spherical roots occurring in a symmetric variety or in a strict variety for a simply laced $G$, it follows that $\mathbb{N} \Sigma$ contains no elements of $\Sigma(G)$ whose double is still in $\Sigma(G)$ (see [8, Table 1]). Therefore, $M^{\prime}$ and $M^{\prime \prime}$ must have the same spherical roots and we get $M^{\prime}=M^{\prime \prime}$, and by Corollary 1.4 it follows that $\Gamma\left(M, \mathcal{L}_{n D}\right) \simeq \Gamma\left(M^{\prime}, \mathcal{L}_{n D}\right)$ for every $n \geqslant 0$. Therefore, we are reduced to the case of a faithful divisor, and the claim follows.

In the adjoint symmetric case, the above proposition was proved in 13 , Theorem 2.6].

If $D_{1}, \ldots, D_{m} \in \mathbb{N} \Delta$, consider the variety

$$
X_{D_{1}, \ldots, D_{m}}=\overline{G \cdot\left(\left[h_{D_{1}}\right] \times \cdots \times\left[h_{D_{m}}\right]\right)} \subset \mathbb{P}\left(V_{D_{1}}^{*}\right) \times \cdots \times \mathbb{P}\left(V_{D_{m}}^{*}\right)
$$

and denote by $\phi_{D_{1}, \ldots, D_{m}}: M \longrightarrow X_{D_{1}, \ldots, D_{m}}$ the map such that $\phi_{D_{1}, \ldots, D_{m}}(x)=$ $\left(\phi_{D_{1}}(x), \ldots, \phi_{D_{m}}(x)\right)$.

Proposition 6.4 ([7, Proposition 1.2]). Let $M$ be a wonderful variety and let $D, E \in \mathbb{N} \Delta$.

i) If $\operatorname{supp}\left(\omega_{D}\right) \cap \operatorname{supp}\left(\omega_{E}\right)=\varnothing$, then $X_{D+E} \simeq X_{D, E}$.

ii) If $M$ is strict and $\operatorname{supp}(D)=\left\{D_{1}, \ldots, D_{m}\right\}$, then $X_{D} \simeq X_{D_{1}, \ldots, D_{m}}$. In particular, if $M$ is strict, $X_{D} \simeq X_{E}$ if and only if $\operatorname{supp}(D)=\operatorname{supp}(E)$.

Proof. i) Since $\operatorname{supp}\left(\omega_{D}\right) \cap \operatorname{supp}\left(\omega_{E}\right)=\varnothing$, by [7, Lemma 1.1] we have a closed equivariant embedding $\psi_{D, E}: \mathbb{P}\left(V_{D}^{*}\right) \times \mathbb{P}\left(V_{E}^{*}\right) \longrightarrow \mathbb{P}\left(V_{D+E}^{*}\right)$, and since $\psi_{D, E}\left(\left[h_{D}\right],\left[h_{E}\right]\right)$ $=\left[h_{D+E}\right]$ the isomorphism $X_{D+E} \simeq X_{D, E}$ follows.

ii) Since $M$ is strict, by the description of the restriction $\omega: \operatorname{Pic}(M) \longrightarrow \mathcal{X}(T)$ (see [42, Lemma 30.24]) it follows that $\operatorname{supp}(D) \cap \operatorname{supp}(E)=\varnothing$ if and only if 
$\operatorname{supp}\left(\omega_{D}\right) \cap \operatorname{supp}\left(\omega_{E}\right)=\varnothing$. Therefore, by [7, Lemma 1.1] we have a closed equivariant embedding $\psi_{D}: \mathbb{P}\left(V_{D_{1}}^{*}\right) \times \cdots \times \mathbb{P}\left(V_{D_{m}}^{*}\right) \longrightarrow \mathbb{P}\left(V_{D}^{*}\right)$ such that $\psi_{D}\left(\left[h_{D_{1}}\right], \ldots,\left[h_{D_{m}}\right]\right)$ $=\left[h_{D}\right]$ and it follows that $X_{D} \simeq X_{D_{1}, \ldots, D_{m}}$.

Assume now that the multiplication of sections is surjective for every couple of globally generated line bundles. In particular $\widetilde{A}(D)$ is generated by its degree one component $\Gamma\left(M, \mathcal{L}_{D}\right)$ and it follows that $A(D)=\widetilde{A}(D)$ if and only if $\Gamma\left(M, \mathcal{L}_{D}\right)=$ $V_{D}$ if and only if $D$ is minuscule or $D=0$. Since $\widetilde{A}(D)$ is integrally closed, we get the following proposition which we need for later use.

Proposition 6.5. Let $M$ be a wonderful variety and suppose that the multiplication of sections is surjective for every couple of globally generated line bundles. Let $D \in \mathbb{N} \Delta, D \neq 0$.

i) If $D$ is minuscule, then $X_{D}$ is projectively normal.

ii) If $\beta_{D}$ is birational and $X_{D}$ is projectively normal, then $D$ is minuscule.

If $D_{1}, \ldots, D_{m} \in \mathbb{N} \Delta$ and if $V_{1}, \ldots, V_{m}$ are $G$-modules of sections such that $V_{D_{i}} \subset V_{i} \subset \Gamma\left(M, \mathcal{L}_{D_{i}}\right)$, consider the associated morphisms $\phi_{V_{i}}: M \longrightarrow \mathbb{P}\left(V_{i}^{*}\right)$ and denote $X_{V_{i}}=\phi_{V_{i}}(M)$. Also, we denote by

$$
X_{V_{1}, \ldots, V_{m}} \subset \mathbb{P}\left(V_{1}^{*}\right) \times \cdots \times \mathbb{P}\left(V_{m}^{*}\right)
$$

the image of $M$ via the map $\phi_{V_{1}, \ldots, V_{m}}(x)=\left(\phi_{V_{1}}(x), \ldots, \phi_{V_{m}}(x)\right)$ and by

$$
X_{V_{1} \otimes \cdots \otimes V_{m}} \subset \mathbb{P}\left(V_{1}^{*} \otimes \cdots \otimes V_{m}^{*}\right)
$$

the image of $X_{V_{1}, \ldots, V_{m}}$ via the Segre embedding.

Lemma 6.6. Let $D_{1}, \ldots, D_{m} \in \mathbb{N} \Delta$ and denote $D=\sum_{i=1}^{m} D_{i}$. If $V_{1}, \ldots, V_{m}$ are $G$-modules such that $V_{D_{i}} \subset V_{i} \subset \Gamma\left(M, \mathcal{L}_{D_{i}}\right)$, then the projective coordinate ring of $X_{V_{1} \otimes \cdots \otimes V_{m}}$ is the subalgebra $A\left(V_{1}, \ldots, V_{m}\right) \subset \widetilde{A}(D)$ generated by the product $V_{1} \cdots V_{m} \subset \Gamma\left(M, \mathcal{L}_{D}\right)$.

Proof. Consider the map $\phi_{V_{1} \otimes \cdots \otimes V_{m}}: M \rightarrow X_{V_{1} \otimes \cdots \otimes V_{m}}$. The lemma follows by noticing that $\phi_{V_{1} \otimes \cdots \otimes V_{m}}^{*} \mathcal{O}(1)=\mathcal{L}_{D}$ and that $\phi_{V_{1} \otimes \cdots \otimes V_{m}}^{*}: V_{1} \otimes \cdots \otimes V_{m} \longrightarrow \Gamma\left(M, \mathcal{L}_{D}\right)$ is the multiplication map.

Proposition 6.7. Let $M$ be a wonderful variety and suppose that the multiplication of sections is surjective for every couple of globally generated line bundles. Let $D_{1}, \ldots, D_{m} \in \mathbb{N} \Delta$ and denote $\Gamma_{i}=\Gamma\left(M, \mathcal{L}_{D_{i}}\right)$. Then the variety $X_{\Gamma_{1} \otimes \cdots \otimes \Gamma_{m}} \subset$ $\mathbb{P}\left(\Gamma_{1}^{*} \otimes \cdots \otimes \Gamma_{m}^{*}\right)$ is projectively normal.

Proof. Denote $D=\sum_{i=1}^{m} D_{i}$. Since the multiplication of sections is surjective, $\Gamma_{1} \cdots \Gamma_{m}=\Gamma\left(M, \mathcal{L}_{D}\right)$, hence by the previous lemma $A\left(\Gamma_{1}, \ldots, \Gamma_{m}\right)=\widetilde{A}(D)$ and $X_{\Gamma_{1} \otimes \cdots \otimes \Gamma_{m}}$ is a projectively normal variety.

Corollary 6.8. Let $M$ be a wonderful variety and suppose that the multiplication of sections is surjective for every couple of globally generated line bundles.

i) Let $D, E \in \mathbb{N} \Delta$ be such that $\operatorname{supp}\left(\omega_{D}\right) \cap \operatorname{supp}\left(\omega_{E}\right)=\varnothing$. If $X_{D}, X_{E}$ are normal, then $X_{D+E}$ is normal as well.

ii) If $M$ is strict, then $X_{D}$ is normal for all $D \in \mathbb{N} \Delta$ if and only if it is normal for all $D \in \Delta$. 
Proof. i) Denote $\Gamma_{D}=\Gamma\left(M, \mathcal{L}_{D}\right)$ and $\Gamma_{E}=\Gamma\left(M, \mathcal{L}_{E}\right)$. By the previous proposition, we have that $X_{\Gamma_{D}, \Gamma_{E}} \simeq X_{\Gamma_{D} \otimes \Gamma_{E}}$ is a normal variety. On the other hand, since $X_{D}$ and $X_{E}$ are normal, we have that $X_{D, E} \simeq X_{\Gamma_{D}, \Gamma_{E}}$, while by Proposition 6.4, $\mathrm{i}$ we have that $X_{D+E} \simeq X_{D, E}$.

ii) Since $M$ is strict, it follows by the description of $\omega: \operatorname{Pic}(M) \longrightarrow \mathcal{X}(T)$ that $\operatorname{supp}\left(\omega_{D}\right) \cap \operatorname{supp}\left(\omega_{E}\right)=\varnothing$ if and only if $\operatorname{supp}(D) \cap \operatorname{supp}(E)=\varnothing$. Therefore the claim follows straightforwardly from i).

Corollary 6.9. Suppose that $M$ is a symmetric variety with reduced root system of type A or that it is a model wonderful variety for a connected semisimple group of type AD. Then $X_{D}$ is normal for all $D \in \mathbb{N} \Delta$.

Proof. By the description of the covering relation, it follows that under the assumptions on $M$ it holds ht $\left(\gamma^{+}\right)=2$ for every covering difference $\gamma$ in $\mathbb{N} \Delta$. Therefore every $D \in \Delta$ is minuscule and $\Gamma\left(M, \mathcal{L}_{D}\right)=V_{D}$. Therefore, $X_{D}$ is projectively normal for all $D \in \Delta$ and it follows by the previous corollary that $X_{D}$ is normal for all $D \in \mathbb{N} \Delta$.

\section{ON THE NORMALITY OF CONES AND NILPOTENT ORBIT CLOSURES}

Following the same approach as in 19 and 13 , we can apply Theorem 4.1 to study the normality of cones over model varieties. In particular, as pointed out by Luna some years ago, we can apply our theory to study the normality of the closure of spherical nilpotent orbits in the Lie algebra of $G$.

Let $M$ be a wonderful variety with set of colors $\Delta$ and set of spherical roots $\Sigma$ and assume that the multiplication of sections is surjective for every couple of globally generated line bundles. Let $D \in \mathbb{N} \Delta$ and denote by $C_{D} \subset V_{D}^{*}$ the cone over the variety $X_{D}$ introduced in the previous section. Analogously, denote by $\widetilde{C}_{D} \subset \Gamma\left(M, \mathcal{L}_{D}\right)^{*}$ the cone over the variety $\widetilde{X}_{D}$. Then the coordinate ring of $C_{D}$ is identified with $A(D)$, whereas that of $\widetilde{C}_{D}$ is identified with $\widetilde{A}(D)$, which is an integrally closed ring. This yields a map $\alpha_{D}: \widetilde{C}_{D} \longrightarrow C_{D}$ that is birational if and only if $\beta_{D}: \widetilde{X}_{D} \longrightarrow X_{D}$ is birational. As already recalled in the previous section, $\widetilde{A}(D)$ is the integral closure of $A(D)$ if and only if $\alpha_{D}$ is birational, whereas $A(D)=\widetilde{A}(D)$ if and only if $D$ is minuscule or $D=0$.

In the case of the model wonderful varieties of simply connected type, after the results of Section 3, we have the following classification of minuscule weights, where $a, b, c \in \mathbb{N}$.

Case $\mathrm{A}_{r}, r$ even: $D_{1}, D_{2}, \ldots, D_{r}, a D_{1}, a D_{r}, a D_{1}+D_{d}$ with $d$ odd, $D_{m}+b D_{r}$ with $m$ even;

Case $\mathrm{A}_{r}, r$ odd: $D_{1}, D_{2}, \ldots, D_{r}, a D_{1}, a D_{r}, a D_{1}+D_{d}$ with $d$ odd, $D_{m}+b D_{r}$ with $m$ odd, $a D_{1}+b D_{r}$;

Case $\mathrm{B}_{r}, r$ even: $a D_{r}, D_{m}+a D_{r}$ with $m$ even;

Case $\mathrm{B}_{r}, r$ odd: $a D_{1}+b D_{r}, a D_{1}+D_{m}+b D_{r}$ with $m$ odd;

Case $\mathrm{C}_{r}: a D_{1}$;

Case $\mathrm{D}_{r}, r$ even: $D_{1}, D_{2}, \ldots, D_{r}, a D_{1}+b D_{r-1}+c D_{r}, a D_{1}+D_{m}+b D_{r-1}+c D_{r}$ with $m$ odd;

Case $\mathrm{D}_{r}, r$ odd: $D_{1}, D_{2}, \ldots, D_{r}, a D_{r-1}+b D_{r}, D_{m}+a D_{r-1}+b D_{r}$ with $m$ even;

Case $\mathrm{E}_{6}: D_{1}, D_{6}, a D_{2}, a D_{2}+D_{3}, a D_{2}+D_{5}$;

Case $\mathrm{E}_{7}: D_{1}, D_{6}, a D_{2}+b D_{7}, a D_{2}+D_{3}+b D_{7}, a D_{2}+D_{5}+b D_{7}$;

Case $\mathrm{E}_{8}: D_{1}, D_{8}, a D_{2}, a D_{2}+D_{3}, a D_{2}+D_{5}, a D_{2}+D_{7}$; 
Case $\mathrm{F}_{4}: a D_{1}$

Case $\mathrm{G}_{2}: a D_{1}$.

Let $\mathfrak{g}$ be the Lie algebra of $G$, as a general reference about nilpotent orbits in $\mathfrak{g}$; see [17. If $\mathfrak{g}$ is not simple, then its nilpotent orbits are products of the nilpotent orbits of its simple factors, and so are their closures. Therefore we may assume that $\mathfrak{g}$ is simple.

Let $e \in \mathfrak{g}$ be a non-zero nilpotent element and let $\mathcal{O}$ be its adjoint orbit. By the Jacobson-Morozov theorem there exists an $\mathfrak{s l}(2)$-triple of the form $(e, h, f)$. Choose a maximal toral subalgebra $\mathfrak{t}$ of $\mathfrak{g}$ containing $h$ and a Borel subalgebra $\mathfrak{b}$ containing $\mathfrak{t}$ and $e$ and such that $\alpha(h) \geqslant 0$ for every $\alpha \in S$, where we denote by $S=\left\{\alpha_{1}, \ldots, \alpha_{r}\right\}$ the set of simple roots defined by the choice of $\mathfrak{t}$ and $\mathfrak{b}$. The string $\left(\alpha_{1}(h), \ldots, \alpha_{r}(h)\right)$ is called the Kostant-Dynkin diagram of $\mathcal{O}$ and it uniquely determines the orbit $\mathcal{O}$. Moreover, every $\alpha_{i}(h)$ is 0,1 or 2 . Let $\theta$ be the highest root corresponding to the choice of $S$ and define the height of $\mathcal{O}$ as height $(\mathcal{O})=\theta(h)$. The height does not depend on the various choices we have made (see [17, $\S 3.5]$ ); furthermore, $\mathcal{O}$ is spherical if and only if $\operatorname{height}(\mathcal{O}) \leqslant 3$, and this last condition is equivalent to saying that it has height equal to 2 or to 3 ; see 38 .

By making use of the projective normality of the symmetric wonderful varieties, in 13 it has been proved that the closure $\overline{\mathcal{O}}$ is normal if height $(\mathcal{O})=2$, which is originally due to W. Hesselink [26]. We now study the normality of $\overline{\mathcal{O}}$ in the case of height $(\mathcal{O})=3$ (see [38, Table 2]) by making use of the projective normality of the model wonderful varieties.

Denote by $\mathcal{U} \simeq G / H$ the orbit of the line $[e] \in \mathbb{P}(\mathfrak{g})=\mathbb{P}(V(\theta))$, namely the image of $\mathcal{O}$ via the natural projection. As every spherical orbit in the projective space of a simple $G$-module, $\mathcal{U}$ possesses a wonderful compactification, which we denote by $M_{\mathcal{O}}$. In [6] we can find a description of the stabilizer of $[e]$ as well as the associated Luna diagram. In particular, $M_{\mathcal{O}}$ turns out to be a strict wonderful variety and, in particular, the restriction of line bundles to the closed orbit $\operatorname{Pic}\left(M_{\mathcal{O}}\right) \longrightarrow \mathcal{X}(B)$ is always injective. Therefore, we may regard $\theta$ as an element of $\mathbb{N} \Delta$ and we have $\overline{\mathcal{U}}=X_{\theta}$ and $\overline{\mathcal{O}}=C_{\theta}$.

In order to study the normality of $\overline{\mathcal{O}}$, we prove the following.

Theorem 7.1. Let $\mathcal{O} \subset \mathfrak{g}$ be a spherical nilpotent orbit and let $M_{\mathcal{O}}$ be the associated wonderful variety. Then the multiplication map

$$
m_{\mathcal{L}, \mathcal{L}^{\prime}}: \Gamma\left(M_{\mathcal{O}}, \mathcal{L}\right) \otimes \Gamma\left(M_{\mathcal{O}}, \mathcal{L}^{\prime}\right) \longrightarrow \Gamma\left(M_{\mathcal{O}}, \mathcal{L} \otimes \mathcal{L}^{\prime}\right)
$$

is surjective for all globally generated line bundles $\mathcal{L}, \mathcal{L}^{\prime}$ on $M_{\mathcal{O}}$.

Notice that by construction $\theta$ is identified with a faithful divisor on $M_{\mathcal{O}}$. Therefore the normality (resp. the non-normality) of $\overline{\mathcal{O}}=C_{\theta}$ follows by noticing case-bycase that $\theta$ is minuscule (resp. non-minuscule) in $\mathbb{N} \Delta$, and applying Proposition 6.5. Given a nilpotent orbit $\mathcal{O}$ of height 3, we summarize the results on the normality of its closure in Table1. Given a spherical nilpotent orbit $\mathcal{O}$, there we write in the second column its Kostant-Dynkin diagram, in the third column its Bala-Carter label (see [15, §8.4]), in the fourth column the corresponding case in [6] and in the fifth column the normality of $\overline{\mathcal{O}}$. In particular we have the following.

Corollary 7.2 (see [18, Theorem 5.1] or [38, Table 2]). Let $\mathcal{O} \subset \mathfrak{g}$ be a spherical nilpotent orbit with height $(\mathcal{O})=3$. Then the closure $\overline{\mathcal{O}}$ is always normal except in cases I and XI of Table 1 . 
TABLE 1. Nilpotent orbits of height 3

\begin{tabular}{|c||c|c|c|c|c|}
\hline & type of $G$ & $\begin{array}{c}\text { Kostant-Dynkin } \\
\text { diagram }\end{array}$ & $\begin{array}{c}\text { partition/ } \\
\text { Bala-Carter label }\end{array}$ & case in [6] & norm. \\
\hline \hline I & $\mathrm{B}_{2 n+1}$ & $(10 \ldots 01)$ & $\left(3,2^{2 n}\right)$ & $(13)$ & no \\
\hline II & $\begin{array}{c}\mathrm{B}_{2 n+m+1} \\
(m>0)\end{array}$ & $(10 \ldots 01 \underbrace{0 \ldots 0}_{m})$ & $\left(3,2^{2 n}, 1^{2 m}\right)$ & $(18)$ & yes \\
\hline III & $\mathrm{D}_{2 n+2}$ & $(10 \ldots 011)$ & $\left(3,2^{2 n}, 1\right)$ & $(41)$ & yes \\
\hline IV & $\begin{array}{c}\mathrm{D}_{2 n+m+2} \\
(m>0)\end{array}$ & $(10 \ldots 01 \underbrace{0 \ldots 0}_{m+1})$ & $\left(3,2^{2 n}, 1^{2 m+1}\right)$ & $(43)$ & yes \\
\hline V & $\mathrm{E}_{6}$ & $(000100)$ & $\mathrm{A}_{1}$ & $(53)$ & yes \\
\hline VI & $\mathrm{E}_{7}$ & $(0010000)$ & $\left(3 \mathrm{~A}_{1}\right)^{\prime}$ & $(54)$ & yes \\
\hline VII & $\mathrm{E}_{7}$ & $(0100001)$ & $4 \mathrm{~A}_{1}$ & $(51)$ & yes \\
\hline VIII & $\mathrm{E}_{8}$ & $(00000010)$ & $3 \mathrm{~A}_{1}$ & $(52)$ & yes \\
\hline IX & $\mathrm{E}_{8}$ & $(01000000)$ & $4 \mathrm{~A}_{1}$ & $(51)$ & yes \\
\hline $\mathrm{X}$ & $\mathrm{F}_{4}$ & $(0100)$ & $\mathrm{A}_{1}+\widetilde{\mathrm{A}}_{1}$ & $(60)$ & yes \\
\hline $\mathrm{XI}$ & $\mathrm{G}_{2}$ & $(10)$ & $\widetilde{\mathrm{A}}_{1}$ & $(66)$ & no \\
\hline
\end{tabular}

The rest of the section is devoted to the proof of Theorem 7.1 and Corollary 7.2 , Since the case height $(\mathcal{O})=2$ was already considered in [13, we consider only the case height $(\mathcal{O})=3$.

7.1. Cases I, III, VII, IX, XI: model orbits. By the description in [6], in these cases we have that $M_{\mathcal{O}}$ is the model wonderful variety of the simply connected group $G$. Notice that $\theta$ is always minuscule in $\mathbb{N} \Delta$ but in the cases $\mathrm{B}_{2 n+1}$ and $\mathrm{G}_{2}$ : it follows that $\overline{\mathcal{O}}$ is normal in the cases III, VII, IX, whereas it is not normal in the cases I, XI.

7.2. Cases IV ( $m$ even), VI, VIII: localization of model wonderful varieties. In this case $M_{\mathcal{O}}$ is not a model wonderful variety, however, it is a quotient of a localization of a model wonderful variety and we still may proceed as in the case of a model orbit thanks to Corollary 1.4 and Corollary 4.14. In order to conclude the argument, we now describe which localizations and quotients we have to take into account in each of the considered cases. We denote by $M$ the model wonderful variety of $G$.

7.2.1. Case IV ( $m$ even). Let $N$ be the boundary divisor corresponding to the spherical root $\sigma_{2 n+1}=\alpha_{2 n+1}+\alpha_{2 n+2}$. Then $M_{\mathcal{O}}$ is the quotient of $N$ by the distinguished subset of colors $\Delta^{\prime}=\left\{D_{2 n+2}, D_{2 n+3}, \ldots, D_{2 n+m+2}\right\}$. Since $\theta=D_{2}$ is minuscule in $\mathbb{N} \Delta$, it follows that $\overline{\mathcal{O}}$ is normal.

7.2.2. Case VI. Let $N$ be the boundary divisor corresponding to the spherical root $\sigma_{3}=\alpha_{3}+\alpha_{4}$. Then $M_{\mathcal{O}}$ is the quotient of $N$ by the distinguished subset $\Delta^{\prime}=$ $\left\{D_{2}, D_{5}, D_{7}\right\}$. Since $\theta=D_{1}$ is minuscule in $\mathbb{N} \Delta$, it follows that $\overline{\mathcal{O}}$ is normal. 
7.2.3. Case VIII. Let $N$ be the boundary divisor corresponding to the spherical root $\sigma_{6}=\alpha_{6}+\alpha_{7}$. Then $M_{\mathcal{O}}$ is the quotient of $N$ by the distinguished subset $\Delta^{\prime}=\left\{D_{2}, D_{3}, D_{4}, D_{5}\right\}$. Since $\theta=D_{8}$ is minuscule in $\mathbb{N} \Delta$, it follows that $\overline{\mathcal{O}}$ is normal.

7.3. Cases II, IV. In these cases we need to prove the surjectivity of the multiplication for other classes of wonderful varieties. Let $G=\operatorname{Spin}(k)$, let $r$ be the semisimple rank of $G$ (i.e., $k=2 r+1$ if $k$ odd or $k=2 r$ if $k$ even) and let $2 \leqslant s \leqslant(k-3) / 2$. Consider the wonderful variety $M$ corresponding in [6] to the case (18) when $k$ is odd and to the case (43) when $k$ is even.

Its spherical roots and colors are given as follows: $\Sigma=\left\{\sigma_{1}, \ldots, \sigma_{s}\right\}$ and $\Delta=$ $\left\{D_{1}, \ldots, D_{s+1}\right\}$, where $\sigma_{i}=\alpha_{i}+\alpha_{i+1}=D_{i}+D_{i+1}-D_{i-1}-D_{i+2}$ for $i=1, \ldots, s-1$ and

$$
\sigma_{s}= \begin{cases}2\left(\alpha_{s+1}+\cdots+\alpha_{r}\right)=2 D_{s+1}-2 D_{s} & \text { if } k \text { is odd } \\ 2\left(\alpha_{s+1}+\cdots+\alpha_{r-2}\right)+\alpha_{r-1}+\alpha_{r}=2 D_{s+1}-2 D_{s} & \text { if } k \text { is even. }\end{cases}
$$

Notice that the Cartan pairing of $M$ does not depend on the parity of $k$. Also, notice that $\omega\left(D_{i}\right)=\omega_{i}$ for $i=1, \ldots, s+1$.

First we need to classify the covering differences for $M$. We omit the proof of the following proposition, which is essentially the same as that of Proposition 3.5 .

Proposition 7.3. Let $\gamma \in \mathbb{N} \Sigma$ be a covering difference in $\mathbb{N} \Delta$ with $\operatorname{supp}_{S}(\gamma)=S$. Then $s$ is even and $\gamma=\sum_{i=1}^{(j-1) / 2} \sigma_{2 i-1}+\sum_{i=(j+1) / 2}^{s / 2} 2 \sigma_{2 i-1}+\sigma_{s}=D_{1}+D_{j}-D_{j-1}$ for $j$ odd $\leqslant s+1$.

In particular, it follows that every covering difference for $M$ satisfies the property (2-ht). Therefore, by Lemma 2.3 , in order to prove the surjectivity of the multiplication of sections of globally generated line bundles on $M$, we are reduced to the study of the low fundamental triples. Here, as in Lemma 4.7, we have the following.

Lemma 7.4. Let $\left(D_{p}, D_{q}, F\right)$ be a low fundamental triple with $\operatorname{supp}_{S}\left(D_{p}+D_{q}-\right.$ $F)=S$. Then $s$ is even, $p, q$ are odd, $p+q \leqslant s+2$ and $F=D_{p+q-2}$.

Proposition 7.5. Let $(D, E, F)$ be a low fundamental triple. Then $s^{D+E-F} V_{F} \subset$ $V_{D} V_{E}$.

Proof. As in the proof of Theorem 4.1 if $\operatorname{supp}(D+E-F) \neq S$ we can proceed by localization and parabolic induction. Therefore it is enough to consider the triples of Lemma 7.4 .

Denote by $U=\mathbb{C}^{k}$ the standard representation of $\operatorname{Spin}(k)$ with the invariant symmetric bilinear form $b$. Let $V \subset U$ be a totally isotropic subspace of dimension $s$, let $\omega_{V} \in \Lambda^{2} V$ be a symplectic form on $V$ and fix $e_{0} \in U \backslash\left(V \oplus V^{*}\right)$ with $b\left(e_{0}, e_{0}\right)=1$.

Let $H$ be the generic stabilizer of $M$. Then $H$ contains the center of $\operatorname{Spin}(k)$ and its image $\bar{H}$ in $\mathrm{SO}(k)$ is described as follows:

$$
\bar{H}=\left\{g \in \mathrm{SO}(k): g e_{0}=e_{0}, \quad g V \subset V, \quad g \omega_{V} \in \mathbb{C} \omega_{V}\right\} .
$$

If $j \leqslant s+1$, then we have $V_{D_{j}}=V\left(\omega_{j}\right)=\Lambda^{j} U$. Let $h_{j}$ be a non-zero $H$-semiinvariant vector in $V_{D_{j}}$. If $2 j \leqslant s$, then up to a scalar factor we have $h_{2 j}=\omega_{V}^{\wedge j}$ 
and $h_{2 j+1}=e_{0} \wedge h_{2 j}$. The projection $\pi: \Lambda^{p} U \otimes \Lambda^{q} U \longrightarrow \Lambda^{p+q-2} U$ is given by contraction as follows:

$\pi\left(\left(v_{1} \wedge \cdots \wedge v_{p}\right) \otimes\left(u_{1} \wedge \cdots \wedge u_{q}\right)\right)=\sum_{i, j}(-1)^{i+j} b\left(v_{i}, u_{j}\right) v_{1} \wedge \cdots \wedge \hat{v}_{i} \wedge \cdots \wedge \hat{u}_{j} \wedge \cdots \wedge u_{q}$.

Therefore, if $\left(D_{p}, D_{q}, D_{p+q-2}\right)$ is as in Lemma 7.4, we get $\pi\left(h_{p} \otimes h_{q}\right)=h_{p+q-2} \neq 0$, and by Lemma 1.2 it follows that $s^{D_{p}+D_{q}-D_{p+q-2}} V_{D_{p+q-2}} \subset V_{D_{p}} V_{D_{q}}$.

If $k=4 n+2 m+3$ and $s=2 n+1$, then $M=M_{\mathcal{O}}$ is the wonderful variety corresponding to the nilpotent orbit $\mathcal{O}$ of the case II, while if $k=4 n+2 m+4$ and $s=2 n+1$, then $M=M_{\mathcal{O}}$ is the wonderful variety corresponding to the nilpotent orbit $\mathcal{O}$ of the case IV. By the previous proposition, the multiplication of sections is surjective for every couple of globally generated line bundles on $M_{\mathcal{O}}$. Since $\theta=D_{2}$ is minuscule in $\mathbb{N} \Delta$, it follows that $\overline{\mathcal{O}}$ is normal.

7.4. Case V. The variety $M_{\mathcal{O}}$ corresponds to the case (53) in [6]. We have $\Sigma=$ $\left\{\sigma_{1}, \sigma_{2}, \sigma_{3}\right\}$ where

$$
\begin{aligned}
& \sigma_{1}=\alpha_{1}+\alpha_{6}=2 D_{1}-D_{3}, \\
& \sigma_{2}=\alpha_{2}+\alpha_{4}=D_{2}-D_{3}+D_{4}, \\
& \sigma_{3}=\alpha_{3}+\alpha_{5}=-D_{1}+2 D_{3}-2 D_{4},
\end{aligned}
$$

and $\omega\left(D_{1}\right)=\omega_{1}+\omega_{6}, \omega\left(D_{2}\right)=\omega_{2}, \omega\left(D_{3}\right)=\omega_{3}+\omega_{5}, \omega\left(D_{4}\right)=\omega_{4}$. The covering differences are

$$
\sigma_{1}, \sigma_{2}, \sigma_{3}, \sigma_{1}+\sigma_{3}, \sigma_{2}+\sigma_{3}, 2 \sigma_{2}+\sigma_{3}, \sigma_{1}+\sigma_{2}+\sigma_{3}
$$

therefore, we have $\operatorname{ht}\left(\sigma^{+}\right)=2$ for every covering difference. Correspondingly, we get the following low fundamental triples:

$$
\begin{gathered}
\left(D_{1}, D_{1}, D_{3}\right),\left(D_{2}, D_{4}, D_{3}\right),\left(D_{3}, D_{3}, D_{1}+2 D_{4}\right),\left(D_{1}, D_{3}, 2 D_{4}\right), \\
\left(D_{2}, D_{3}, D_{1}+D_{4}\right),\left(D_{2}, D_{2}, D_{1}\right),\left(D_{1}, D_{2}, D_{4}\right) .
\end{gathered}
$$

We need to show that $s^{E+D-F} V_{F} \subset V_{D} V_{E}$ for all low fundamental triples $(D, E, F)$. As in the proof of Theorem 4.1 if $\operatorname{supp}(D+E-F) \neq S$ we can proceed by localization and parabolic induction. In this way, the triples $\left(D_{1}, D_{1}, D_{3}\right)$, $\left(D_{3}, D_{3}, D_{1}+2 D_{4}\right),\left(D_{1}, D_{3}, 2 D_{4}\right)$ reduce to the case of a symmetric wonderful variety, the triple $\left(D_{2}, D_{4}, D_{3}\right)$ reduces to a model wonderful variety and the triples $\left(D_{2}, D_{3}, D_{1}+D_{4}\right),\left(D_{2}, D_{2}, D_{1}\right)$ reduce to a wonderful variety studied in the case IV. So we are left to study the cases $D=D_{1}, E=D_{2}$ and $F=D_{4}$, which can easily be checked via computer (as explained in Section 4.5). Since $\theta=D_{2}$ is minuscule in $\mathbb{N} \Delta$, it follows that $\overline{\mathcal{O}}$ is normal.

7.5. Case X. The variety $M_{\mathcal{O}}$ corresponds to the case (60) in 6]. We have $\Sigma=$ $\left\{\sigma_{1}, \sigma_{2}, \sigma_{3}\right\}$ where

$$
\begin{aligned}
& \sigma_{1}=2 \alpha_{4}=2 D_{1}-D_{3}, \\
& \sigma_{2}=\alpha_{1}+\alpha_{2}=D_{2}-D_{3}+D_{4}, \\
& \sigma_{3}=2 \alpha_{3}=-D_{1}+2 D_{3}-2 D_{4},
\end{aligned}
$$

and $\omega\left(D_{1}\right)=2 \omega_{4}, \omega\left(D_{2}\right)=\omega_{2}, \omega\left(D_{3}\right)=2 \omega_{3}, \omega\left(D_{4}\right)=\omega_{1}$. Since the Cartan pairing of $M_{\mathcal{O}}$ is the same as that of the previous case, it follows that the covering differences and the low fundamental triples are also the same. 
In order to prove that $s^{E+D-F} V_{F} \subset V_{D} V_{E}$ for all low fundamental triples $(D, E, F)$, if $\operatorname{supp}(D+E-F) \neq S$ we can proceed by localization and parabolic induction. In this way, the triples $\left(D_{1}, D_{1}, D_{3}\right),\left(D_{3}, D_{3}, D_{1}+2 D_{4}\right),\left(D_{1}, D_{3}, 2 D_{4}\right)$ reduce to the case of a symmetric wonderful variety, the triple $\left(D_{2}, D_{4}, D_{3}\right)$ reduces to a model wonderful variety and the triples $\left(D_{2}, D_{3}, D_{1}+D_{4}\right),\left(D_{2}, D_{2}, D_{1}\right)$ reduce to a wonderful variety studied in the case III. The only remaining cases, $D=D_{1} E=D_{2}$ and $F=D_{4}$, can easily be checked via computer (as explained in Section 4.5). Since $\theta=D_{4}$ is minuscule in $\mathbb{N} \Delta$, it follows that $\overline{\mathcal{O}}$ is normal.

\section{Application to the real model orbit of type $\mathrm{E}_{8}$}

In [1, Adams, Huang and Vogan study the model orbit in the Lie algebra of type $\mathrm{E}_{8}$. Their study is motivated by the so-called orbit method to construct representations of reductive Lie groups. In their case the group is the complex algebraic group of type $\mathrm{E}_{8}$ considered as a real Lie group. In particular they describe the decomposition into irreducible modules of the coordinate ring of the nilpotent orbit of type IX (see Section 7) and prove it is indeed a model orbit. In the same paper they also make some conjectures about another orbit which is the analogue of the model orbit for the split real form of $E_{8}$.

We start with some general preliminaries, we refer to [1] and to [43] for the motivation of these constructions coming from the representation theory of Lie groups. Let $\tilde{G}_{\mathbb{R}}$ be a real form of a connected and complex algebraic semisimple group $\tilde{G}$ and let $\sigma$ be the associated Galois involution of $\tilde{G}$. There exists a complex algebraic involution $\theta$ of $\tilde{G}$ which commutes with $\sigma$ such that the subgroup $K_{\mathbb{R}}$ of points of $\tilde{G}_{\mathbb{R}}$ fixed by $\theta$ is a maximal compact subgroup of $\tilde{G}_{\mathbb{R}}$. Then the subgroup $K$ of points of $\tilde{G}$ fixed by $\theta$ is a complexification of $K_{\mathbb{R}}$. The Lie algebra $\tilde{\mathfrak{g}}$ of $\tilde{G}$ decomposes as $\mathfrak{k} \oplus \mathfrak{p}$ where $\mathfrak{k}$ is the Lie algebra of $K$ and $\mathfrak{p}$ is the eigenspace of eigenvalue -1 of $\theta$. An analogue of the nilpotent cone $\mathcal{N}$ is defined as

$$
\mathcal{N}_{\theta}=\mathcal{N} \cap \mathfrak{p} .
$$

Fix a point $e \in \mathcal{N}_{\theta}$, let $\mathcal{O}$ be its $K$-orbit and $K(e)$ its stabilizer. Consider the multiplicative character $\gamma_{e}$ of $K(e)$ given by $\gamma_{e}(g)=\operatorname{det}\left(\left.\operatorname{Ad}_{g}\right|_{\mathfrak{k}(e)}\right) \operatorname{det}\left(\left.\operatorname{Ad}_{g}\right|_{\mathfrak{k}}\right)^{-1}$. If $\chi: K(e) \longrightarrow \mathbb{C}^{*}$ is any multiplicative character we can consider the algebraic line bundle on $\mathcal{O}$ given by $\mathcal{V}_{\chi}=K \times_{K(e)} \mathbb{C}_{\chi}$. As in [1, the pair $(e, \chi)$ is said to be admissible if $\overline{\mathcal{O}} \backslash \mathcal{O}$ has codimension at least two in $\overline{\mathcal{O}}$ and $\chi^{2}(g)=\gamma_{e}(g)$ for all $g$ in the identity component of $K(e)$. In this paper we need a slightly more general definition of admissible pair.

Let $G$ be a double covering of $K$ and $G(e)$ be the inverse image of $K(e)$ in $G$ so that $\mathcal{O} \simeq G / G(e)$. Let $\gamma_{e}^{\prime}$ denote the character of $G(e)$ induced by $\gamma_{e}$. Given a character $\chi$ of $G(e)$ we can construct the line bundle $\mathcal{V}_{\chi}$ as above. We say that the pair $(e, \chi)$ is admissible if $\overline{\mathcal{O}} \backslash \mathcal{O}$ has codimension at least two in $\overline{\mathcal{O}}$ and $\chi^{2}(g)=\gamma_{e}^{\prime}(g)$ for all $g$ in the identity component of $G(e)$. Let $G_{\mathbb{R}}$ also be the inverse image of $K_{\mathbb{R}}$ in $G$. Notice that $G$ is the complexification of $G_{\mathbb{R}}$. The coverings of $\tilde{G}_{\mathbb{R}}$ are in correspondence with the coverings of $K_{\mathbb{R}}$ hence there exists a Lie group $\hat{G}_{\mathbb{R}}$ which is a double covering of $\tilde{G}_{\mathbb{R}}$ whose maximal compact subgroup is $G_{\mathbb{R}}$.

Assume that $R(e, \chi)$ is the irreducible unitary representation of $\hat{G}_{\mathbb{R}}$, attached to an admissible pair $(e, \chi)$ according to the orbit method. Then the decomposition of the $G_{\mathbb{R}}$-finite vectors of $R(e, \chi)$ into irreducible submodules should be equal to 
the decomposition into irreducible $G$-submodules of the space of algebraic sections $\Gamma\left(\mathcal{O}, \mathcal{V}_{\chi}\right)$ (see [1, Conjecture 2.9]).

In [1], Adams, Huang and Vogan analyze the geometric side of two particular cases of this construction. They obtain a complete description of the decomposition of $\Gamma\left(\mathcal{O}, \mathcal{V}_{\chi}\right)$ into $G$-modules in one of these cases and a conjectural description in the second case. In Section 8.1 we recall the result obtained by Adams, Huang and Vogan in the first case and we analyze it using our techniques. In Sections 8.3, 8.4 and 8.6 we analyze the second case considered by Adams, Huang and Vogan and we prove that their conjectural description of $\Gamma\left(\mathcal{O}, \mathcal{V}_{\chi}\right)$ is correct.

8.1. The case of the complex model orbit. Let $\tilde{G}_{\mathbb{R}}$ be the complex algebraic group of type $\mathrm{E}_{8}$. Hence $\tilde{G}=\tilde{G}_{\mathbb{R}} \times \tilde{G}_{\mathbb{R}}$ and $G=K$ is the diagonal subgroup. So $\mathfrak{p}$ is isomorphic to the Lie algebra of $G$ and one can consider the nilpotent orbit $\mathcal{O}$ with Kostant-Dynkin diagram equal to $(0,1,0,0,0,0,0,0)$ (case IX of Section 7). In this case $\gamma_{e}$ is trivial and the stabilizer $K(e)$ is connected, so $\chi$ also has to be trivial and $\Gamma\left(\mathcal{O}, \mathcal{V}_{\chi}\right)=\mathbb{C}[\mathcal{O}]$. In [1] it is proved that all simple modules of $G$ appear in $\mathbb{C}[\mathcal{O}]$ with multiplicity one.

We fix a maximal torus $T$, and a Borel subgroup of $G$ containing $T$. We denote by $\Phi$ the set of roots and by $S$ the set of simple roots determined by these choices. We denote also by $\varepsilon_{1}, \ldots, \varepsilon_{8}$ an orthonormal basis of $\mathcal{X}(T)$ such that $\Phi$ and $S$ have the following description (with respect to the choice given in [1] we have changed the sign of $\left.\varepsilon_{1}\right)$ :

$$
\begin{aligned}
\Phi & =A \cup B \text { where } A=\left\{ \pm \varepsilon_{i} \pm \varepsilon_{j}: i \neq j\right\} \text { and } \\
B & =\left\{\frac{1}{2}\left(\sum_{i=1}^{8} a_{i} \varepsilon_{i}\right): a_{i}= \pm 1 \text { and } \prod_{i=1}^{8} a_{i}=1\right\} ; \\
S & =\left\{\alpha_{1}, \ldots, \alpha_{8}\right\} \text { where } \alpha_{1}=-\varepsilon_{1}-\varepsilon_{2}, \\
\alpha_{2} & =\frac{1}{2}\left(\varepsilon_{1}-\varepsilon_{2}-\varepsilon_{3}+\varepsilon_{4}+\varepsilon_{5}+\varepsilon_{6}+\varepsilon_{7}+\varepsilon_{8}\right) \text { and } \\
\alpha_{i} & =\varepsilon_{i-1}-\varepsilon_{i} \text { for } i=3, \ldots, 8 .
\end{aligned}
$$

For each root $\alpha$ choose also an $\mathfrak{s l}(2)$-triple $x_{\alpha}, \alpha^{\vee}, y_{\alpha}$ where $x_{\alpha}$ has weight $\alpha$ and $y_{\alpha}$ has weight $-\alpha$. Let $\beta_{1}=-\varepsilon_{1}+\varepsilon_{2}, \beta_{2}=\varepsilon_{3}+\varepsilon_{4}, \beta_{3}=\varepsilon_{5}+\varepsilon_{6}, \beta_{4}=\varepsilon_{7}+\varepsilon_{8}$ and define

$$
\begin{gathered}
e_{0}=x_{\beta_{1}}+x_{\beta_{2}}+x_{\beta_{3}}+x_{\beta_{4}}, \quad f_{0}=y_{\beta_{1}}+y_{\beta_{2}}+y_{\beta_{3}}+y_{\beta_{4}} \\
h_{0}=\beta_{1}^{\vee}+\beta_{2}^{\vee}+\beta_{3}^{\vee}+\beta_{4}^{\vee}=-\varepsilon_{1}^{*}+\varepsilon_{2}^{*}+\cdots+\varepsilon_{8}^{*} .
\end{gathered}
$$

These elements are an $\mathfrak{s l}(2)$-triple and it is clear that $e_{0}$ is an element with associated Kostant-Dynkin diagram equal to $(0,1,0,0,0,0,0,0)$. Let $\mathcal{O}$ be its orbit. As we have already recalled in Section 7 , the orbit of the line spanned by $e_{0}$ in $\mathbb{P}(\mathfrak{g})$ is the open orbit of the model wonderful variety of $\mathrm{E}_{8}$.

The colors of the model wonderful variety of $\mathrm{E}_{8}$ are $D_{1}, \ldots, D_{8}$ with $\omega\left(D_{i}\right)=\omega_{i}^{\alpha}$, where $\omega_{1}^{\alpha}, \ldots, \omega_{8}^{\alpha}$ are the fundamental weights w.r.t. the simple system $\alpha_{1}, \ldots, \alpha_{8}$. Notice also that, in the notation of the previous section, we have $\overline{\mathcal{O}}=C_{D_{8}}$. Finally, the spherical roots of $M$ are

$$
\begin{gathered}
\sigma_{1}=\alpha_{6}+\alpha_{7}, \quad \sigma_{2}=\alpha_{4}+\alpha_{5}, \quad \sigma_{3}=\alpha_{1}+\alpha_{3}, \quad \sigma_{4}=\alpha_{2}+\alpha_{4}, \\
\sigma_{5}=\alpha_{5}+\alpha_{6}, \quad \sigma_{6}=\alpha_{3}+\alpha_{4}, \quad \sigma_{7}=\alpha_{7}+\alpha_{8} .
\end{gathered}
$$


Here we have ordered them so that it is clear that they are a basis of a root system of type $D_{7}$. Notice also that, since in this case $\omega$ is injective, the list above also determines the Cartan pairing $c$ of the wonderful variety $M$.

Theorem 8.1 (see [1, Theorem 1.1]). The variety $\overline{\mathcal{O}}$ is normal and

$$
\mathbb{C}[\overline{\mathcal{O}}]=\mathbb{C}[\mathcal{O}] \simeq \bigoplus_{\lambda \in \mathcal{X}(T)^{+}} V(\lambda)
$$

Proof. We know that $\overline{\mathcal{O}}$ is normal by the discussion in Section 7 , in particular, we have $\overline{\mathcal{O}}=C_{D_{8}}=\widetilde{C}_{D_{8}}$. Moreover, each adjoint orbit has even dimension so the first equality follows by normality. Since $\overline{\mathcal{O}}=\widetilde{C}_{D_{8}}$ we also have

$$
\mathbb{C}[\overline{\mathcal{O}}] \simeq \bigoplus_{n \geqslant 0} \Gamma\left(M, \mathcal{L}_{n D_{8}}\right) \simeq \bigoplus_{\substack{n \geqslant 0, \gamma \in \mathbb{N} \Sigma: \\ n D_{8}-\gamma \in \mathbb{N} \Delta}} s^{\gamma} V_{n D_{8}-\gamma}
$$

Now notice that $D_{8}$ and the spherical roots are linearly independent and that $\omega$ is injective in this case, so all irreducible $G$-representations occur with multiplicity at most one. Moreover, since the variety is irreducible, if $V(\lambda)$ and $V(\mu)$ occur in this decomposition then $V(\lambda+\mu)$ also occurs. Finally, we have

$$
\begin{aligned}
& D_{1}=2 D_{8}-\left(\sigma_{4}+2 \sigma_{5}+\sigma_{6}+2 \sigma_{7}\right), \\
& D_{2}=4 D_{8}-\left(\sigma_{1}+2 \sigma_{2}+3 \sigma_{3}+4 \sigma_{4}+6 \sigma_{5}+3 \sigma_{6}+5 \sigma_{7}\right), \\
& D_{3}=5 D_{8}-\left(\sigma_{1}+2 \sigma_{2}+3 \sigma_{3}+5 \sigma_{4}+7 \sigma_{5}+3 \sigma_{6}+6 \sigma_{7}\right), \\
& D_{4}=7 D_{8}-\left(\sigma_{1}+2 \sigma_{2}+4 \sigma_{3}+6 \sigma_{4}+9 \sigma_{5}+4 \sigma_{6}+8 \sigma_{7}\right), \\
& D_{5}=6 D_{8}-\left(\sigma_{1}+2 \sigma_{2}+4 \sigma_{3}+6 \sigma_{4}+8 \sigma_{5}+4 \sigma_{6}+7 \sigma_{7}\right), \\
& D_{6}=4 D_{8}-\left(\sigma_{2}+2 \sigma_{3}+3 \sigma_{4}+4 \sigma_{5}+2 \sigma_{6}+4 \sigma_{7}\right), \\
& D_{7}=3 D_{8}-\left(\sigma_{2}+2 \sigma_{3}+3 \sigma_{4}+4 \sigma_{5}+2 \sigma_{6}+3 \sigma_{7}\right), \\
& D_{8}=D_{8}-0,
\end{aligned}
$$

hence $V\left(\omega_{i}\right)$ occurs in the decomposition of $\mathbb{C}[\overline{\mathcal{O}}]$, for all $i$.

As we have already recalled above, the second isomorphism in the statement of the theorem was already proved in [1. Notice that our proof of this isomorphism does not use the projective normality proved in Section 4 but only general considerations on wonderful varieties.

The normality of $\overline{\mathcal{O}}$ was not studied in $[1$, however, Panyushev in the last section of 38 sketches how to deduce it by their result. Notice that our proof of the normality of $\overline{\mathcal{O}}$, relies on Theorem 4.1 for which, in the case of $\mathrm{E}_{8}$, we used a computer. The normality of $\overline{\mathcal{O}}$ is due to A. Broer (see [12, Section 7.8, Remark iii)]), however, we could not find a proof in the literature.

8.2. From complex to real orbits: general considerations. Let $\tilde{G}, K, \tilde{\mathfrak{g}}, \mathfrak{p}$ be as at the beginning of this section. Let $\mathcal{O} \subset \tilde{\mathfrak{g}}$ be a nilpotent adjoint orbit of the complex algebraic group $\tilde{G}$. We want to make some general standard remarks on the intersection $\mathcal{O} \cap \mathfrak{p}$ (see for example [40, Section 9]). Fix $e \in \mathcal{O} \cap \mathfrak{p}$ and let $\tilde{G}(e)$ and $K(e)$ be the stabilizers of $e$ in $\tilde{G}$ and $K$, respectively. The subgroup $\tilde{G}(e)$ is stable under $\theta$ and we define $Z=\left\{z \in \tilde{G}(e): \theta(z)=z^{-1}\right\}$. We have an action of $\tilde{G}(e)$ on $Z$ by $g \cdot z=g z \theta(g)^{-1}$ and we define $\mathbb{H}^{1}$ as $Z$ modulo the action of $\tilde{G}(e)$. 
Lemma 8.2. i) Every connected component of $\mathcal{O} \cap \mathfrak{p}$ is a single $K$-orbit.

ii) The map $g \cdot x \mapsto g^{-1} \theta(g)$ induces a bijection from the set of $K$-orbits in $\mathcal{O} \cap \mathfrak{p}$ to $\mathbb{H}^{1}$.

iii) Every connected component of $Z$ is a single $\tilde{G}(e)^{0}$-orbit (where $\tilde{G}(e)^{0}$ is the identity component of $\tilde{G}(e))$.

Proof. Set $\eta=-\theta$. First notice that $\mathcal{O} \cap \mathfrak{p}=\mathcal{O}^{\eta}$. Being $\mathcal{O}$ smooth and $\eta$ an involution we deduce that $\mathcal{O}^{\eta}$ is smooth. Take $e \in \mathcal{O}^{\eta}$. We prove that

$$
(\tilde{\mathfrak{g}} \cdot e) \cap \mathfrak{p}=\mathfrak{k} \cdot e .
$$

Let $\tilde{\mathfrak{g}}(e)$ be the annihilator of $e$ in $\tilde{\mathfrak{g}}$ and let $y \in \tilde{\mathfrak{g}}$ be such that $y \cdot e \in \mathfrak{p}$. Then $\theta(y \cdot e)=-y \cdot e$, hence $z=\theta(y)-y \in \tilde{\mathfrak{g}}(e)$. Take $u=\frac{1}{2} z$, then $u \in \tilde{\mathfrak{g}}(e)$ is such that $v=y+u \in \mathfrak{k}$ and $v \cdot e=y \cdot e$.

This proves that any $K$-orbit in $\tilde{G} \cdot e \cap \mathfrak{p}$ is open. This implies $i)$.

Point $i i i$ ) can be proved similarly and $i i$ ) is trivial.

In particular, notice that if we prove that $Z$ is connected then it follows that $\mathcal{O} \cap \mathfrak{p}$ is a single $K$-orbit.

We refine this lemma, using the Jordan decomposition. Choose an $\mathfrak{s l}(2)$-triple, $e, h, f$ such that $\theta(h)=h$ and $\theta(f)=-f$, this is always possible; see 32, Proposition 4]. Let $U$ be the unipotent radical of $\tilde{G}(e)$ and $L=\{g \in \tilde{G}(e): g \cdot h=h\}$. Then $\tilde{G}(e)=L \cdot U$ is a Levi decomposition of $\tilde{G}(e)$ (see [3, Proposition 2.4]). Notice also that $L$ and $U$ are stable under the action of $\theta$. Define $Z_{L}=Z \cap L$ and $\mathbb{H}_{L}^{1}$ as $Z_{L}$ modulo the action of $L$ given by $g \cdot z=g z \theta(g)^{-1}$.

Lemma 8.3. i) The inclusion $Z_{L} \subset Z$ induces a bijection from $\mathbb{H}_{L}^{1}$ to $\mathbb{H}^{1}$.

ii) If $Z_{L}$ is connected, then $\mathcal{O} \cap \mathfrak{p}$ is a single $K$-orbit.

Proof. Let $x, y \in Z_{L}$ and assume that $x=h y \theta(h)^{-1}$ with $h=\ell u, \ell \in L$ and $u \in U$. It follows immediately that $x=\ell y \theta(\ell)^{-1}$. This prove the injectivity of the map $\mathbb{H}_{L}^{1} \longrightarrow \mathbb{H}^{1}$ induced by the inclusion $Z_{L} \subset Z$.

Now let $z=\ell u \in Z$ with $\ell \in L$ and $u \in U$. From $\theta(z)=z^{-1}$ we deduce that $\theta(\ell)=\ell^{-1}$ and $\theta(u)=\ell u^{-1} \ell^{-1}$. Now $U$ being unipotent there exists a unique $v \in U$ such that $v^{-2}=u$. Using again that $U$ is unipotent we deduce that $\theta(v)=\ell v^{-1} \ell^{-1}$. Set $h^{\prime}=\ell v \ell^{-1}$, then

$$
h^{\prime}(\ell u) \theta\left(h^{\prime}\right)^{-1}=\ell v \ell^{-1} \ell u \ell^{-1} \ell v \ell^{-1} \ell=\ell .
$$

Hence the map $\mathbb{H}_{L}^{1} \longrightarrow \mathbb{H}^{1}$ is surjective. This proves $i$ ). Now $\left.i i\right)$ follows from Lemma 8.2 .

8.3. The case of the real model orbit of $E_{8}$. In the last two sections of [1] a real version of the model orbit is considered. We now recall from [1 some of the structural results about this orbit. We also prove Proposition 8.5 which is probably well known and is somehow implicit (even if not necessary) in the discussion in 1 .

From now on $\tilde{G}$ is the complex algebraic group of type $\mathrm{E}_{8}$ and $\tilde{G}_{\mathbb{R}}$ is its split real form. Then $K$ is isogenous to $\operatorname{Spin}(16)$. For the complex group of type $\mathrm{E}_{8}$ we have already introduced some notation in Section 8.1 (notice that this group had the role of the group $G$ and not of $\tilde{G}$ in that section). We keep that notation, so $T$ is a maximal torus of $\tilde{G}, \Phi$, the associated root system, and $\varepsilon_{1}, \ldots, \varepsilon_{8}, A, B$, $S=\left\{\alpha_{1}, \ldots, \alpha_{8}\right\}$ are as in Section 8.1 
Then we can choose $\tilde{G}_{\mathbb{R}}$ so that $\mathfrak{k}$ is the Lie algebra spanned by $\mathfrak{t}$, the Lie algebra of $T$, and by the vectors $x_{\alpha}$ with $\alpha \in A$. With this choice $\mathfrak{p}$ is spanned by the vectors $x_{\alpha}$ with $\alpha \in B$.

As a simple system for the root system of $\mathfrak{k} \simeq \mathfrak{s o}(16)$ we choose the usual basis, but we enumerate it starting from zero, that is,

$$
\tau_{i}=\varepsilon_{i+1}-\varepsilon_{i+2} \text { for } i=0, \ldots, 6 \text { and } \tau_{7}=\varepsilon_{7}+\varepsilon_{8} .
$$

We denote by $\omega_{i}^{\mathrm{D}}$ the associated fundamental weights. In particular we obtain that $\mathfrak{p}$ is the spin representation associated to the weight $\omega_{7}^{\mathrm{D}}$. Moreover, since $\tilde{G}$ is simply connected the subgroup $K$ is connected. Finally, notice that $\omega_{6}^{\mathrm{D}} \notin \mathcal{X}(T)$ while $\omega_{7}^{\mathrm{D}} \in \mathcal{X}(T)$. Hence $\operatorname{Spin}(16)$ is a double covering of $K$. We set $G=\operatorname{Spin}(16)$. Notice that in [1] it is claimed that $K$ is isomorphic to $\operatorname{Spin}(16)$, but this does not affect any of their arguments.

In order to prove that the roots $\tau_{1}, \ldots, \tau_{7}$ are conjugated to the roots $\sigma_{1}, \ldots, \sigma_{7}$ introduced in Section 8.1, we introduce a new simple system of the root system of type $\mathrm{E}_{8}$. The vectors

$$
\begin{array}{lll}
\zeta_{1}=\frac{1}{2}\left(-\varepsilon_{2}-\varepsilon_{3}-\varepsilon_{4}+\varepsilon_{5}\right), & \zeta_{2}=\frac{1}{2}\left(\varepsilon_{1}+\varepsilon_{6}+\varepsilon_{7}-\varepsilon_{8}\right), \\
\zeta_{3}=\frac{1}{2}\left(\varepsilon_{2}+\varepsilon_{3}-\varepsilon_{4}+\varepsilon_{5}\right), & \zeta_{4}=\frac{1}{2}\left(\varepsilon_{1}+\varepsilon_{6}-\varepsilon_{7}+\varepsilon_{8}\right), \\
\zeta_{5}=\frac{1}{2}\left(\varepsilon_{2}-\varepsilon_{3}+\varepsilon_{4}+\varepsilon_{5}\right), & \zeta_{6}=\frac{1}{2}\left(\varepsilon_{1}-\varepsilon_{6}+\varepsilon_{7}+\varepsilon_{8}\right), \\
\zeta_{7}=\frac{1}{2}\left(-\varepsilon_{2}+\varepsilon_{3}+\varepsilon_{4}+\varepsilon_{5}\right), & \zeta_{8}=\frac{1}{2}\left(\varepsilon_{1}-\varepsilon_{6}-\varepsilon_{7}-\varepsilon_{8}\right),
\end{array}
$$

form an orthonormal basis of $\mathfrak{t}^{*}$, and $\pm \zeta_{i} \pm \zeta_{j} \in \Phi$ for $i$ odd and $j$ even. Notice also that

$$
\begin{aligned}
\gamma_{2} & =\frac{1}{2}\left(\zeta_{1}-\zeta_{2}-\zeta_{3}+\zeta_{4}+\zeta_{5}+\zeta_{6}+\zeta_{7}+\zeta_{8}\right) \\
& =\frac{1}{2}\left(\varepsilon_{1}-\varepsilon_{2}-\varepsilon_{3}+\varepsilon_{4}+\varepsilon_{5}-\varepsilon_{6}-\varepsilon_{7}+\varepsilon_{8}\right) \in \Phi, \\
\gamma_{1} & =-\zeta_{1}-\zeta_{2}, \text { and } \gamma_{i}=\zeta_{i-1}-\zeta_{i}, \text { for } i=3, \ldots, 8,
\end{aligned}
$$

form a simple system of $\Phi$ (since they are elements of $\Phi$ with the right scalar products). Hence there exists an element $w$ in the Weyl group such that $w\left(\varepsilon_{i}\right)=\zeta_{i}$, for all $i$. Finally, notice that we have

$$
\begin{gathered}
\tau_{1}=\gamma_{6}+\gamma_{7}, \quad \tau_{2}=\gamma_{4}+\gamma_{5}, \quad \tau_{3}=\gamma_{1}+\gamma_{3}, \quad \tau_{4}=\gamma_{2}+\gamma_{4}, \\
\tau_{5}=\gamma_{5}+\gamma_{6}, \quad \tau_{6}=\gamma_{3}+\gamma_{4}, \quad \tau_{7}=\gamma_{7}+\gamma_{8},
\end{gathered}
$$

so that $w\left(\sigma_{i}\right)=\tau_{i}$. Notice also that

$$
\tau_{0}=\frac{1}{2}\left(\zeta_{1}-\zeta_{3}-\zeta_{5}+\zeta_{7}+\zeta_{2}+\zeta_{4}+\zeta_{6}+\zeta_{8}\right)=\gamma_{2}+\gamma_{3}+\gamma_{4}+\gamma_{5}
$$

We denote by $\Lambda$ the weight lattice of $\operatorname{Spin}(16)$ and by $\Lambda_{\mathrm{E}}=\mathcal{X}(T)$ the sublattice given by the weights of $\mathrm{E}_{8}$. We denote also by $\Lambda^{+}$the dominant weights of $\Lambda$ w.r.t. the simple system $\tau_{0}, \ldots, \tau_{7}$ and by $\Lambda_{\mathrm{E}}^{+}$the dominant weights of $\Lambda_{\mathrm{E}}$ w.r.t. the simple system $\gamma_{1}, \ldots, \gamma_{8}$. We have $\Lambda_{\mathrm{E}}^{+} \subset \Lambda^{+}$. We denote by $\omega_{i}^{\gamma}$ the fundamental weights of $\Lambda_{\mathrm{E}}$ w.r.t. the simple system $\gamma_{1}, \ldots, \gamma_{8}$. Notice that $\omega_{7}^{\mathrm{D}}=\omega_{8}^{\gamma}$.

Now let $\mathcal{O}$ be the adjoint orbit of $\tilde{\mathfrak{g}}$ considered in Section 8.1. We want to study the intersection $\mathcal{O} \cap \mathfrak{p}$. Let us first choose an element $e \in \mathcal{O} \cap \mathfrak{p}$.

Consider the semisimple part $K_{0}$ of the standard Levi factor of the maximal parabolic subgroup $P_{0}$ of $K$ associated with $\tau_{0}$. This is a group isogeneous to Spin(14), we denote its Lie algebra by $\mathfrak{k}_{0}$. Let $\mathfrak{p}_{0}$ be the subspace of $\mathfrak{p}$ spanned 
by the root vectors of weight of the form $\frac{1}{2}\left(\sum a_{i} \varepsilon_{i}\right)$ with $a_{1}=-1$. Its highest weight is $\frac{1}{2}\left(-\varepsilon_{1}+\varepsilon_{2}+\varepsilon_{3}+\varepsilon_{4}+\varepsilon_{5}+\varepsilon_{6}+\varepsilon_{7}-\varepsilon_{8}\right)$. Hence it is an irreducible Spin(14)-submodule of $\mathfrak{p}$ isomorphic to the module $V_{D_{8}}^{*}$ of Section 5.5.3.

Lemma 8.4. The vector $h_{8}$ of Section 5.5 .3 belongs to $\mathcal{O}$.

Proof. The vectors

$$
\begin{array}{ll}
\eta_{1}=\frac{1}{2}\left(\varepsilon_{1}-\varepsilon_{6}-\varepsilon_{7}-\varepsilon_{8}\right), & \eta_{2}=\frac{1}{2}\left(-\varepsilon_{2}+\varepsilon_{3}+\varepsilon_{4}+\varepsilon_{5}\right), \\
\eta_{3}=\frac{1}{2}\left(-\varepsilon_{1}+\varepsilon_{6}-\varepsilon_{7}-\varepsilon_{8}\right), & \eta_{4}=\frac{1}{2}\left(\varepsilon_{2}-\varepsilon_{3}+\varepsilon_{4}+\varepsilon_{5}\right), \\
\eta_{5}=\frac{1}{2}\left(-\varepsilon_{1}-\varepsilon_{6}+\varepsilon_{7}-\varepsilon_{8}\right), & \eta_{6}=\frac{1}{2}\left(\varepsilon_{2}+\varepsilon_{3}-\varepsilon_{4}+\varepsilon_{5}\right), \\
\eta_{7}=\frac{1}{2}\left(-\varepsilon_{1}-\varepsilon_{6}-\varepsilon_{7}+\varepsilon_{8}\right), & \eta_{8}=\frac{1}{2}\left(\varepsilon_{2}+\varepsilon_{3}+\varepsilon_{4}-\varepsilon_{5}\right)
\end{array}
$$

form an orthonormal basis of $\mathfrak{t}^{*}$, and $\pm \eta_{i} \pm \eta_{j} \in \Phi$ if $i$ is odd and $j$ is even. Notice also that

$$
\begin{gathered}
\hat{\gamma}_{2}=\frac{1}{2}\left(\eta_{1}-\eta_{2}-\eta_{3}+\eta_{4}+\eta_{5}+\eta_{6}+\eta_{7}+\eta_{8}\right)=\varepsilon_{2}-\varepsilon_{6}, \\
\hat{\gamma}_{1}=-\eta_{1}-\eta_{2}, \text { and } \hat{\gamma}_{i}=\eta_{i-1}-\eta_{i}, \text { for } i=3, \ldots, 8
\end{gathered}
$$

is a simple system of $\Phi$. Hence there exists an element $w$ of the Weyl group such that $w\left(\varepsilon_{i}\right)=\eta_{i}$, for all $i$. Choose a representative $\dot{w}$ of $w$ in $\tilde{G}$. Define $e=\dot{w}\left(e_{0}\right)$, $h=w\left(h_{0}\right)=-\eta_{1}^{*}+\eta_{2}^{*}+\cdots+\eta_{8}^{*}$ and $f=\dot{w}\left(f_{0}\right)$. Then $\theta(h)=h$ and $\theta(f)=-f$. More explicitly, we have $h=-2 \varepsilon_{1}^{*}+\varepsilon_{2}^{*}+\varepsilon_{3}^{*}+\varepsilon_{4}^{*}+\varepsilon_{5}^{*}, e=x_{\delta_{1}}+x_{\delta_{2}}+x_{\delta_{3}}+x_{\delta_{4}}$, where $w\left(\beta_{1}\right)=\delta_{1}=-\eta_{1}+\eta_{2}, w\left(\beta_{2}\right)=\delta_{2}=\eta_{3}+\eta_{4}, w\left(\beta_{3}\right)=\delta_{3}=\eta_{5}+\eta_{6}$, $w\left(\beta_{4}\right)=\delta_{4}=\eta_{7}+\eta_{8}$ and similarly for $f$.

Notice that $e \in \mathfrak{p}_{0}$. Moreover, the two vectors $e$ and $h_{8}$ are linear combinations with non-zero coefficients of vectors of the same weights $\delta_{1}, \delta_{2}, \delta_{3}, \delta_{4}$ and these weights are linearly independent, so they are conjugated under the action of the maximal torus.

Let $w \in W$ be the element defined in the previous proof, as shown there we can choose a representative $\dot{w}$ of $w$ such that $\dot{w}\left(e_{0}\right)=h_{8}$. Set $e=h_{8}$ and $h=w\left(h_{0}\right)$. In particular, the stabilizer of $e$ is the parabolic induction of the stabilizer of $h_{8}$ in $\operatorname{Spin}(14)$. More explicitly, we have

$$
\mathfrak{k}(e)=\mathbb{C} \omega_{0}^{\vee} \oplus \mathfrak{u}_{0}^{-} \oplus \mathfrak{h}_{0},
$$

where $\mathfrak{u}_{0}^{-}$is the Lie algebra of the unipotent radical of the parabolic subgroup opposite to $P_{0}, \mathfrak{h}_{0}$ is the annihilator of $h_{8}$ in $\mathfrak{k}_{0}$ and $\omega_{0}^{\vee}$ is orthogonal to $\tau_{1}, \ldots, \tau_{7}$. In particular, the Levi factor of $\mathfrak{k}(e)$ is isomorphic to $\mathfrak{g l}(4)$.

We now want to describe in some detail the stabilizer $K(e)$ and apply Lemma 8.3 to prove that $\mathcal{O} \cap \mathfrak{p}$ is a single $K$-orbit. As recalled in Section 8.1, the Levi factor $\tilde{L}$ of $\tilde{G}(e)$ is $\operatorname{Sp}(8)$ and $L=\tilde{L}^{\theta}$. Furthermore, notice that there is only one involution of $\mathrm{Sp}(8)$ such that the Lie algebra of the fixed point subgroup is isomorphic to $\mathfrak{g l}(4)$. This involution can be described as follows. Let $I_{4} \in \mathrm{SL}(4)$ be the identity matrix, and define the $8 \times 8$ matrices

$$
J=\left(\begin{array}{cc}
0 & I_{4} \\
-I_{4} & 0
\end{array}\right), \quad \rho=\left(\begin{array}{cc}
I_{4} & 0 \\
0 & -I_{4}
\end{array}\right),
$$

and $\operatorname{Sp}(8)$ as the matrices preserving the form $J$. Then $\operatorname{Sp}(8)$ is stable under the conjugation by the matrix $\rho$, which is an involution. Moreover, $L$ is isomorphic to GL(4). 
Proposition 8.5. If $\mathcal{O}$ is the model orbit of $\mathrm{E}_{8}$ and $\tilde{G}_{\mathbb{R}}$ is the split real form of $\mathrm{E}_{8}$, then $\mathcal{O} \cap \mathfrak{p}$ is a single $K$-orbit.

Proof. By Lemma 8.3 and the above discussion, it is enough to prove that

$$
Z_{L}=\left\{z \in \operatorname{Sp}(8): \theta(z)=z^{-1}\right\}
$$

is connected. Let $z=\left(\begin{array}{cc}A & B \\ C & D\end{array}\right)$ where $A, B, C, D$ are $4 \times 4$ matrices. The condition $z \in Z_{L}$ is equivalent to

$$
A^{2}=I_{4}+B C, \quad D=A^{t}, \quad B=B^{t}, \quad C=C^{t}, \quad A B=B A^{t} \text { and } A^{t} C=C A .
$$

Acting by $g \in \operatorname{Sp}(8)$, via $g \cdot z=g z \theta(g)^{-1}$, we remain in the same connected component. Using $g$ of the form $\left(\begin{array}{cc}\alpha & 0 \\ 0 & \alpha^{-1}\end{array}\right)$ with $\alpha \in \mathrm{GL}(4)$ we see that we can assume $B$ is of the form $\left(\begin{array}{cc}I_{r} & 0 \\ 0 & 0\end{array}\right)$ and $A=\left(\begin{array}{ll}a & b \\ c & d\end{array}\right)$, and using equations (8.3) we see that $c=0, a=a^{t}$ and $d^{2}=1$. Then using $g$ of the forms $\left(\begin{array}{cc}\beta & 0 \\ 0 & \beta^{-1}\end{array}\right)$ and $\beta=\left(\begin{array}{cc}I_{r} & 0 \\ 0 & \gamma\end{array}\right)$ we can also assume that $d$ is diagonal. Now we choose $g$ of the form $\left(\begin{array}{cc}I & s I \\ 0 & I\end{array}\right)$. We get

$$
g \cdot z=\left(\begin{array}{cc}
A+s C & B+s\left(A+A^{t}\right)+s^{2} C \\
C & A^{t}+s C
\end{array}\right) .
$$

If we compute the determinant of $B+s\left(A+A^{t}\right)+s^{2} C$ we see that it is a polynomial in $s$ and its lowest degree term is $4 \operatorname{det}(d)$. Hence there exists $s$ such that $B+s(A+$ $\left.A^{t}\right)+s^{2} C$ is invertible. So we can assume $B$ invertible and arguing as before we can assume $B=I_{4}$. Now, for $B=I_{4}$, the equations in (8.3) take the form $A=A^{t}=D$, and $C=-I+A^{2}$. Such equations define an algebraic subset which is isomorphic to an affine space and, in particular, connected. Therefore $Z_{L}$ is connected.

8.4. The coordinate ring of the real model orbit. Here we describe the coordinate ring of $\mathcal{O} \cap \mathfrak{p}$. In [1] it is shown that this description follows from the vanishing of certain cohomology groups which in the case of the real model orbit is conjectural (see Conjecture 3.13 and Theorem 7.13 in [1).

Let $M_{0}$ be the wonderful comodel variety of cotype $\mathrm{E}_{8}$. This is a wonderful variety for the group $\operatorname{Spin}(14)$. Consider the parabolic induction $M$ of $M_{0}$ to $\operatorname{Spin}(16)$. This is a wonderful variety with spherical roots equal to $\tau_{1}, \ldots, \tau_{7}$ and colors $D_{0}^{\prime}, \ldots, D_{8}^{\prime}$, where $\omega\left(D_{0}^{\prime}\right)=\omega_{0}^{\mathrm{D}}$ and, for $i>0, D_{i}^{\prime}$ is induced by the respective color of $M_{0}$ (see Section 5 ).

Let $\mathcal{O}_{\mathfrak{p}}=\mathcal{O} \cap \mathfrak{p}$. Then $X_{\mathfrak{p}}=\mathbb{P}\left(\mathcal{O}_{\mathfrak{p}}\right) \subset \mathbb{P}(\mathfrak{p})$ is the open orbit of $M$, and $\overline{\mathcal{O}_{\mathfrak{p}}}$ is the cone $C_{D}$ for $D=D_{8}^{\prime}$. Indeed, $e=h_{8} \in V_{\omega\left(D_{8}^{\prime}\right)}^{*}=V_{\omega_{7}^{\mathrm{D}}}^{*}=V_{\omega_{7}^{\mathrm{D}}} \simeq \mathfrak{p}$ and $D_{8}^{\prime}$ is faithful.

Theorem 8.6. The cone $\overline{\mathcal{O}_{\mathfrak{p}}}$ is normal and we have the following isomorphism of K-modules:

$$
\mathbb{C}\left[\overline{\mathcal{O}_{\mathfrak{p}}}\right] \simeq \bigoplus_{\lambda \in \Lambda_{\mathrm{E}}^{+}} V(\lambda) .
$$

Proof. Notice that the combinatorics of colors and spherical roots is essentially the same as that of the model wonderful variety of type $\mathrm{E}_{8}$. In particular, since $D_{8}^{\prime}$ is minuscule, from Theorem 5.2 it follows that $\overline{\mathcal{O}_{\mathfrak{p}}}$ is normal. Hence, as a $K$-module we have that its coordinate ring is the sum of all the modules $V_{n D_{8}^{\prime}-\tau}$ with $n \geqslant 0$, $\tau \in \mathbb{N}\left[\tau_{1}, \ldots, \tau_{7}\right]$ and $n D_{8}^{\prime}-\tau \in \mathbb{N}\left[D_{0}^{\prime}, \ldots, D_{8}^{\prime}\right]$. Moreover, with $\tau$ as above, the latter condition is equivalent to $n D_{8}^{\prime}-\tau \in \mathbb{N}\left[D_{1}^{\prime}, \ldots, D_{8}^{\prime}\right]$. Now notice that $\omega\left(D_{8}^{\prime}\right)$ and $\tau_{1}, \ldots, \tau_{7}$ are linearly independent (this is not true for $M_{0}$ but it is true for $M$ 
because of the presence of the extra color $\left.D_{0}^{\prime}\right)$. So we obtain that the coordinate ring of $\overline{\mathcal{O}_{\mathfrak{p}}}$ is the sum of all modules $V\left(n \omega_{8}^{\mathrm{D}}-\tau\right)$ where $n$ and $\tau$ are as above. Finally, the computation is exactly the same as that given in the proof of Theorem 8.1 for the model orbit of type $\mathrm{E}_{8}$, since the two situations are conjugated by an element of the Weyl group.

Notice that our proof of the normality of $\overline{\mathcal{O}_{\mathfrak{p}}}$ via Theorem 5.2 did not require any computer calculation. Moreover, the description of the coordinate ring of the normalization of $\overline{\mathcal{O}_{\mathfrak{p}}}$ is independent of Theorem 5.2

The combinatorics of distinguished subsets of colors allows us to describe completely the $K$-orbits in the closure of $\mathcal{O}_{\mathfrak{p}}$ (see for example 22]), and in particular to prove that $\overline{\mathcal{O}_{\mathfrak{p}}} \backslash \mathcal{O}_{\mathfrak{p}}$ has codimension at least two in $\overline{\mathcal{O}_{\mathfrak{p}}}$. Indeed, one sees that this property depends only on the combinatorics of colors and spherical roots, and in this case the combinatorics is the same as that of the complex model orbit, whose boundary has codimension at least two. More precisely, here $\operatorname{codim}_{\overline{\mathcal{O}}}(\overline{\mathcal{O}} \backslash \mathcal{O})=16$ and $\operatorname{codim}_{\overline{\mathcal{O}_{\mathfrak{p}}}}\left(\overline{\mathcal{O}_{\mathfrak{p}}} \backslash \mathcal{O}_{\mathfrak{p}}\right)=8$.

Here we can avoid such an argument. Below we prove that $\mathbb{C}\left[\mathcal{O}_{\mathfrak{p}}\right]=\mathbb{C}\left[\overline{\mathcal{O}_{\mathfrak{p}}}\right]$ and this also implies that $\overline{\mathcal{O}_{\mathfrak{p}}} \backslash \mathcal{O}_{\mathfrak{p}}$ has codimension at least two.

8.5. Computation of the space of sections of a line bundle associated to an admissible pair: general considerations. For the next lemma we put ourselves in a general setting. Let $G$ be simply connected and let $M$ be a wonderful compactification of $G / H$. Let $E \in \mathbb{N} \Delta$. Let $\mathbb{C} h$ be a line in $V\left(\omega_{E}^{*}\right)$ where $H$ acts with character $\xi_{E}$. Assume that the stabilizer of $\mathbb{C} h$ is equal to $H$ and let $H_{0}$ be the stabilizer of $h$. Furthermore, assume that $\xi_{E}$ induces an isomorphism of $H / H_{0}$ with $\mathbb{C}^{*}$ : we identify $H / H_{0}$ with $\mathbb{C}^{*}$ choosing such an isomorphism. Finally, notice that $H / H_{0}$ acts on the right on $G / H_{0}$.

Lemma 8.7. Let $D \in \mathbb{Z} \Delta$ and let $\chi$ denote the restriction of $\xi_{D}$ to $H_{0}$. We have the following isomorphism of $G$-modules

$$
\Gamma\left(G / H_{0}, \mathcal{V}_{\chi}\right) \simeq \bigoplus_{\substack{n \in \mathbb{Z}, \sigma \in \mathbb{Z} \Sigma: \\ D+n E-\sigma \in \mathbb{N} \Delta}} V_{D+n E-\sigma}
$$

Proof. We have

$$
\Gamma\left(G / H_{0}, \mathcal{V}_{\chi}\right) \simeq\left(\mathbb{C}[G] \otimes \mathbb{C}_{-\chi}\right)^{H_{0}} \simeq \bigoplus_{\lambda \in \mathcal{X}(T)^{+}} V\left(\lambda^{*}\right) \otimes\left(V(\lambda) \otimes \mathbb{C}_{-\chi}\right)^{H_{0}}
$$

Now notice that $H$ acts on $\left(V(\lambda) \otimes \mathbb{C}_{-\chi}\right)^{H_{0}}$ and so the latter decomposes into $H$ stable subspaces where $H$ acts by a character of the form $\xi_{D}+n \xi_{E}$. Moreover, all these eigenspaces are of dimension one, since $H$ is spherical. Hence we have

$$
\Gamma\left(G / H_{0}, \mathcal{V}_{\chi}\right) \simeq \bigoplus_{\lambda \in \mathcal{X}(T)^{+}, n \in \mathbb{Z}} V\left(\lambda^{*}\right) \otimes\left(V(\lambda) \otimes \mathbb{C}_{-\xi_{D}-n \xi_{E}}\right)^{H}
$$

Now recall that the space of spherical vectors $V\left(\omega_{F}^{*}\right)_{\xi_{F}}^{(H)}$ is non-zero if and only if $F \in \mathbb{N} \Delta$, and $\xi_{F}=\xi_{D+n E}$ if and only if $F=D+n E-\sigma$ for $\sigma \in \mathbb{Z} \Sigma$.

Let us specialize this identity to the case $D=0$ and compare the coordinate ring of $G / H_{0}$ with the coordinate ring of the normalization of its closure in $V\left(\omega_{E}^{*}\right)$. 
Since

$$
\mathbb{C}\left[\widetilde{C}_{E}\right]=\bigoplus_{n \geqslant 0} \Gamma\left(M, \mathcal{L}_{n E}\right) \simeq \bigoplus_{\substack{n \geqslant 0, \sigma \in \mathbb{N} \Sigma: \\ n E-\sigma \in \mathbb{N} \Delta}} V_{n E-\sigma},
$$

we have the following.

Lemma 8.8. The equality $\mathbb{C}\left[G / H_{0}\right]=\mathbb{C}\left[\widetilde{C}_{E}\right]$ is equivalent to the fact that, for all $n \in \mathbb{Z}$ and $\sigma \in \mathbb{Z} \Sigma$, if $n E-\sigma \in \mathbb{N} \Delta$ then $n \geqslant 0$ and $\sigma \in \mathbb{N} \Sigma$.

We now apply this lemma to our special case in which $G$ is $\operatorname{Spin}(16), H_{0}=G(e)$, $M$ is the wonderful compactification of $X_{\mathfrak{p}}$ and $E=D_{8}^{\prime}$.

Lemma 8.9. For all $n \in \mathbb{Z}$ and $\sigma \in \mathbb{Z} \Sigma$, if $n D_{8}^{\prime}-\sigma \in \mathbb{N} \Delta$ then $n \geqslant 0$ and $\sigma \in \mathbb{N} \Sigma$.

Proof. Write $n D_{8}^{\prime}-\sigma=\sum_{i=0}^{8} a_{i} D_{i}^{\prime}$ and notice that the conditions $a_{1}, \ldots, a_{8} \geqslant 0$ are equivalent to the conditions obtained from Theorem 8.1 by applying Lemma 8.8 to the complex model orbit of type $\mathrm{E}_{8}$.

From this and Theorem 8.6 by applying Lemma 8.8 to the real model orbit, we get that

$$
\mathbb{C}\left[\mathcal{O}_{\mathfrak{p}}\right]=\mathbb{C}\left[\overline{\mathcal{O}_{\mathfrak{p}}}\right]
$$

8.6. Sections of the line bundle associated to the admissible pair for the real model orbit. We now want to compute the characters $\gamma_{e}$ and $\gamma_{e}^{\prime}$. For this we further analyze the stabilizer $T(e)$ of $e$ in $T$. Let $\Lambda_{\mathrm{E}}^{\vee}$ be the lattice dual to $\Lambda_{\mathrm{E}}$. Recall the definition of the vectors $\eta_{i}$ given in the equations (8.1) and denote by $\eta_{i}^{*}$ the vectors of the dual basis. Let $x_{1}^{*}=\eta_{1}^{*}+\eta_{2}^{*}$ and $x_{i}^{*}=\eta_{2 i-1}^{*}-\eta_{2 i}^{*}$ for $i=2,3,4$ and define $R^{\vee}$ as the sublattice of $\Lambda_{\mathrm{E}}^{\vee}$ generated by $x_{1}^{*}, \ldots, x_{4}^{*}$ and $R_{0}^{\vee}$ as the sublattice generated by $x_{1}^{*}+x_{2}^{*}, x_{1}^{*}+x_{3}^{*}, x_{1}^{*}+x_{4}^{*}$. Then $R^{\vee}$ and $R_{0}^{\vee}$ are direct factors of $\Lambda_{\mathrm{E}}^{\vee}$. Finally, let $T_{0}$ be the maximal torus of $K_{0}$ (the subgroup of $K$ isogenous to $\operatorname{Spin}(14)$ introduced above) contained in $T$ such that $T_{0}(e)=T(e) \cap T_{0}$. We have

$$
T=\Lambda_{\mathrm{E}}^{\vee} \otimes_{\mathbb{Z}} \mathbb{C}^{*}, \quad T(e)=R^{\vee} \otimes_{\mathbb{Z}} \mathbb{C}^{*} \quad \text { and } \quad T_{0}(e)=R_{0}^{\vee} \otimes_{\mathbb{Z}} \mathbb{C}^{*} .
$$

We already know that the Levi factor of $K(e)$ is isomorphic to GL(4) so that any character is a power of the determinant. We describe now the center of GL(4) and the determinant as an element of dual lattice of $R^{\vee}$. Let $z^{*}=x_{1}^{*}-x_{2}^{*}-x_{3}^{*}-x_{4}^{*}=$ $2 \varepsilon_{1}^{*}+\varepsilon_{2}^{*}+\varepsilon_{3}^{*}+\varepsilon_{4}^{*}+\varepsilon_{5}^{*}$, then using the description of $\mathfrak{h}_{0}$ given in Section [5.5] it is easy to see that $z^{*}$ is a central cocharacter. In particular, if $R_{Z}^{\vee}=\mathbb{Z} z^{*}$ then

$$
T_{Z}=R_{Z}^{\vee} \otimes_{\mathbb{Z}} \mathbb{C}^{*}
$$

is the center of $L \simeq \mathrm{GL}(4)$. Now we compute the character $\gamma_{e}$. We have already noticed that it is enough to compute its restriction to $T$. Moreover, its restriction to $T_{0}(e)$ must be trivial since $\mathrm{SL}(4)$ has no characters so we compute its restriction to $T_{Z}$. Using the description of the stabilizer $\mathfrak{k}(e)$ in (8.2) and the description of $\mathfrak{h}_{0}$ given in Section 5.5 it is easy to prove that $\left\langle\gamma_{e}, z^{*}\right\rangle=4$. Hence we see that $\gamma_{e}$ restricted to the Levi of $K(e)$, which is isomorphic to GL(4), equals the determinant and

$$
\gamma_{e}=x_{1}-x_{2}-x_{3}-x_{4}
$$

In particular, it is not the square of a character. However, on the covering $G$ of $K$ we can consider the character $-\omega_{0}^{\mathrm{D}}=-\varepsilon_{1}$, and we denote by $\chi$ its restriction to $G(e)$. We have

$$
\chi=\frac{1}{2}\left(x_{1}-x_{2}-x_{3}-x_{4}\right)=\frac{1}{2} \gamma_{e}^{\prime} .
$$


Notice that $\xi_{D_{0}^{\prime}}=\chi$. Indeed, $\omega_{D_{0}^{\prime}}=\omega_{0}^{\mathrm{D}}$ and $G(e)$ contains the unipotent radical $U_{0}^{-}$of $P_{0}^{-}$. Hence the only $G(e)$-semi-invariant in $V\left(\omega_{0}^{\mathrm{D}}\right)$ is the lowest weight vector which has weight $-\omega_{0}^{\mathrm{D}}$.

We now describe the space of sections $\Gamma\left(\mathcal{O}_{\mathfrak{p}}, \mathcal{V}_{\chi}\right)$. In [1] the same description follows from a vanishing result which is not proved in this case (see Conjecture 8.6 and Theorem 8.10 in [1]).

Theorem 8.10. We have the following isomorphism of $G$-modules:

$$
\Gamma\left(\mathcal{O}_{\mathfrak{p}}, \mathcal{V}_{\chi}\right) \simeq \bigoplus_{\lambda \in \Lambda_{\mathrm{E}}^{+}} V\left(\omega_{0}^{\mathrm{D}}+\lambda\right)
$$

Proof. We have seen above that $\xi_{D_{0}^{\prime}}=\chi$. Hence we can apply Lemma 8.7 with $D=D_{0}^{\prime}$ and $E=D_{8}^{\prime}$. We obtain

$$
\Gamma\left(\mathcal{O}_{\mathfrak{p}}, \mathcal{V}_{\chi}\right) \simeq \bigoplus_{\substack{n \in \mathbb{Z}, \sigma \in \mathbb{Z} \Sigma: \\ D_{0}^{\prime}+n D_{8}^{\prime}-\sigma \in \mathbb{N} \Delta}} V_{D_{0}^{\prime}+n D_{8}^{\prime}-\sigma}
$$

Write $D_{0}^{\prime}+n D_{8}^{\prime}-\sigma=\sum_{i=0}^{8} a_{i} D_{i}^{\prime}$. Notice that as in the proof of Lemma 8.9 this implies $a_{1}, \ldots, a_{8} \geqslant 0$. Hence we obtain $n \geqslant 0$ and $\sigma \in \mathbb{N} \Sigma$. In particular, the condition $a_{0} \geqslant 0$ is automatically satisfied. As already noticed in the proof of Theorem 8.6. $\Lambda_{\mathrm{E}}^{+}$consists of the weights of the form $\omega\left(n D_{8}^{\prime}-\sigma\right)$ for $n \geqslant 0, \sigma \in \mathbb{N} \Sigma$ and $n D_{8}^{\prime}-\sigma \in \mathbb{N} \Delta$.

\section{Degeneracy of the multiplication}

Here we give a counterexample to the surjectivity of the multiplication of sections of line bundles, on wonderful varieties, generated by global sections.

9.1. Counterexample. Let $G$ be $\mathrm{SO}(2 r+1)$ and let $M$ be the model wonderful variety for the group $G$. It is not isomorphic to the model wonderful variety for $\operatorname{Spin}(2 r+1)$. Its set of colors $\Delta=\left\{D_{1}, \ldots, D_{r}\right\}$ is in bijection, via $\omega$, with the set $\left\{\omega_{1}, \ldots, \omega_{r-1}, 2 \omega_{r}\right\}$. Its set of spherical roots is $\left\{\alpha_{1}+\alpha_{2}, \ldots, \alpha_{r-1}+\alpha_{r}, 2 \alpha_{r}\right\}$. If $r=2$ or $r=3$, then the multiplication of sections is surjective. Set $r=4$ and consider the low triple $\left(D_{2}, D_{2}, D_{1}\right)$; then $V\left(\omega_{1}\right) \not \subset V\left(\omega_{2}\right)^{\otimes 2}$, hence $s^{2 D_{2}-D_{1}} V_{D_{1}} \not \subset$ $V_{D_{2}}^{2}$. In particular, the multiplication of sections is not surjective, for all $r \geqslant 4$.

9.2. Degeneracy of the multiplication. Roughly speaking, in its nature the previous example does not express a lack of the multiplication, but rather a lack of the tensor product. Indeed, $V\left(\omega_{1}\right) \not \subset V\left(\omega_{2}\right)^{\otimes 2}$ but $V\left(2 \omega_{1}\right) \subset V\left(2 \omega_{2}\right)^{\otimes 2}$, so that it expresses the fact that the saturation property does not hold for groups of type B: similar things cannot happen if $G$ is of type A and, conjecturally, whenever $G$ is simply laced. It is worth noticing that the same situation holds if we consider the multiplication of the wonderful variety considered in a previous example: $s^{2 D_{2}-D_{1}} V_{D_{1}} \not \subset V_{D_{2}}^{2}$ but $s^{4 D_{2}-2 D_{1}} V_{2 D_{1}} \subset V_{2 D_{2}}^{2}$.

We briefly recall what the saturation property is. Let $G$ be a simply connected almost simple algebraic group. We say that the saturation property holds for $G$ if, whenever $d>0$ and $\lambda, \mu, \nu \in \Lambda^{+}$are such that $\nu \leqslant \lambda+\mu$ and $V(d \nu) \subset$ $V(d \lambda) \otimes V(d \mu)$, then it also holds that $V(\nu) \subset V(\lambda) \otimes V(\mu)$. In [31] A. Knutson and T. Tao showed that the saturation property holds if $G$ is of type A, while in 28. M. Kapovich and J. Millson conjectured that the saturation property holds whenever $G$ is simply laced. 
We want to consider the saturation property in the more general context of the multiplication law attached to a wonderful variety, the classical case corresponding to the wonderful compactification of an adjoint group ([27, Lemma 3.1]). We say that the saturation property holds for a wonderful variety $M$ with set of colors $\Delta$ and set of spherical roots $\Sigma$ if, whenever $d>0$ and $D, E, F \in \mathbb{N} \Delta$ are such that $D \leqslant \Sigma E+F$ and $s^{d(E+F-D)} V_{d D} \subset V_{d E} V_{d F}$, then the inclusion $s^{E+F-D} V_{D} \subset V_{E} V_{F}$ also holds.

Suppose that $M$ is a wonderful variety and let $E, F \in \mathbb{N} \Delta$. Then the following inclusion holds:

$$
V_{E} V_{F} \subset \bigoplus_{\substack{D \in \mathbb{N} \Delta: D \leqslant \Sigma E+F \\ V\left(\omega_{D}\right) \subset V\left(\omega_{E}\right) \otimes V\left(\omega_{F}\right)}} s^{E+F-D} V_{D} .
$$

If the equality holds in the previous inclusion, then we say that the product $V_{E} V_{F}$ is non-degenerate, while if the equality holds for every $E, F \in \mathbb{N} \Delta$ then we say that the multiplication of $M$ is non-degenerate. It is easy to show that if the multiplication of $M$ is non-degenerate and if the saturation property holds for $G$, then the latter holds for $M$ as well.

Another equivalent description of the multiplication follows by identifying sections of line bundles on $M$ with $H$-semi-invariant functions on $G, H$ acting on the right. More precisely, given $E, F \in \mathbb{N} \Delta$, we may identify $\Gamma\left(M, \mathcal{L}_{E}\right), \Gamma\left(M, \mathcal{L}_{F}\right)$ and $\Gamma\left(M, \mathcal{L}_{E+F}\right)$ with submodules of $\mathbb{C}[G]^{(H)}$, and the multiplication of sections $\Gamma\left(M, \mathcal{L}_{E}\right) \otimes \Gamma\left(M, \mathcal{L}_{F}\right) \longrightarrow \Gamma\left(M, \mathcal{L}_{E+F}\right)$ coincides with the restriction of the multiplication defined in $\mathbb{C}[G]^{(H)}$. Therefore we regard the modules $V_{E}, V_{F}, V_{E} V_{F}$ inside $\mathbb{C}[G]^{(H)}$, and the multiplication is the multiplication of functions.

Example 1. Let $G=\mathrm{SL}(2)$ and consider the basic case of the rank one wonderful variety $M=\mathbb{P}^{1} \times \mathbb{P}^{1}$, whose generic stabilizer is the maximal torus $T$. Then $\Sigma=\{\alpha\}$ is the unique simple root of $G$ and $\Delta=\left\{D^{+}, D^{-}\right\}$, where $D^{+}=\mathbb{P}^{1} \times\{[1,0]\}$ and $D^{-}=\{[1,0]\} \times \mathbb{P}^{1}$. Moreover $\alpha=D^{+}+D^{-}$and $\omega_{D^{+}}=\omega_{D^{-}}=1$ equal the fundamental weight of $G$.

Given $n \in \mathbb{N}$, identify the simple $G$-module $V(n)$ of highest weight $n$ with $\mathbb{C}[x, y]_{n}$, the set of homogeneous polynomials of degree $n$ in two variables. Given $a D^{+}+b D^{-} \in \mathbb{N} \Delta$, the corresponding $T$-eigenvector is $h(a, b)=x^{a} y^{b}$.

The projection $\pi_{m}: V(a+b) \otimes V(c+d) \longrightarrow V(a+b+c+d-2 m)$ is described on the basis of $T$-eigenvectors as follows:

$$
x^{a} y^{b} \otimes x^{c} y^{d} \longmapsto \sum_{u+v=m}(-1)^{u} u ! v !\left(\begin{array}{l}
a \\
u
\end{array}\right)\left(\begin{array}{l}
b \\
v
\end{array}\right)\left(\begin{array}{l}
c \\
v
\end{array}\right)\left(\begin{array}{l}
d \\
u
\end{array}\right) x^{a+c-m} y^{b+d-m} .
$$

As shown by the following two examples, the multiplication of $M$ is degenerate.

- Consider $D^{+}+D^{-} \leqslant_{\Sigma} 2\left(D^{+}+D^{-}\right)$. Then $V(2) \subset V(2)^{\otimes 2}$ but $\pi_{1}(h(1,1) \otimes$ $h(1,1))=0$, so that $V_{D^{+}+D^{-}} \not \subset V_{D^{+}+D^{-}}^{2}$. This can also be explained since $V(2)$ is not contained in the second symmetric power of $V(2)$.

- Consider $2 D^{+}+D^{-} \leqslant \Sigma\left(3 D^{+}+D^{-}\right)+\left(D^{+}+2 D^{-}\right)$. Then $V(3) \subset V(4) \otimes$ $V(3)$ but $\pi_{2}(h(3,1) \otimes h(1,2))=0$, so that $V_{2 D^{+}+D^{-}} \not \subset V_{3 D^{+}+D^{-}} V_{D^{+}+2 D^{-}}$.

More generally, the multiplication is degenerate whenever $\Sigma \cap S \neq \varnothing$. This can be reduced to the basic case of SL(2)/T as shown in the following proposition.

Proposition 9.1. Suppose that $S \cap \Sigma \neq \varnothing$. Then the multiplication is degenerate. 
Proof. Let $\alpha \in S \cap \Sigma$ and suppose that the multiplication is non-degenerate. Consider the rank one wonderful subvariety $M^{\prime}$ defined by intersecting all the $G$-stable prime divisors $M^{\sigma}$ with $\sigma \in \Sigma \backslash\{\alpha\}$, denote $\Delta^{\prime}$ its set of colors. Then $M^{\prime}$ is the quotient of a parabolic induction of a wonderful variety for SL(2) isomorphic to $\mathbb{P}^{1} \times \mathbb{P}^{1}$, whose generic stabilizer is a maximal torus of $\mathrm{SL}(2)$ and whose set of colors is identified with $\Delta^{\prime}(\alpha)=\left\{D \in \Delta^{\prime}: P_{\alpha} D \neq D\right\}$, where $P_{\alpha}$ denotes the minimal parabolic associated to $\alpha$. It follows then by Corollary 1.7 that, for every $D^{\prime} \in \mathbb{N}\left[\Delta^{\prime} \backslash \Delta^{\prime}(\alpha)\right], V_{D^{\prime}} V_{E^{\prime}}=V_{D^{\prime}+E^{\prime}}$ for all $E^{\prime} \in \mathbb{N} \Delta^{\prime}$.

Let $D^{\prime}, E^{\prime}, F^{\prime} \in \mathbb{N} \Delta^{\prime}$ be such that $D^{\prime} \leqslant \Sigma^{\prime} E^{\prime}+F^{\prime}$ and $V\left(\omega_{D^{\prime}}\right) \subset V\left(\omega_{E^{\prime}}\right) \otimes V\left(\omega_{F^{\prime}}\right)$. Denote $\rho: \operatorname{Pic}(M) \longrightarrow \operatorname{Pic}\left(M^{\prime}\right)$ the restriction. From the combinatorics of colors and spherical roots it follows that there exist $E_{0}^{\prime}, F_{0}^{\prime} \in \mathbb{N}\left[\Delta^{\prime} \backslash \Delta^{\prime}(\alpha)\right]$ and $E, F \in \mathbb{N} \Delta$ such that $E^{\prime}+E_{0}^{\prime}=\rho(E)$ and $F^{\prime}+F_{0}^{\prime}=\rho(F)$. Let $D=E+F-\left(E^{\prime}+F^{\prime}-D^{\prime}\right)$ and notice that $D \in \mathbb{N} \Delta$ and $D^{\prime}+E_{0}^{\prime}+F_{0}^{\prime}=\rho(D)$. Moreover, the inclusion $V\left(\omega_{D^{\prime}}\right) \subset V\left(\omega_{E^{\prime}}\right) \otimes V\left(\omega_{F^{\prime}}\right)$ implies that $V\left(\omega_{D}\right) \subset V\left(\omega_{E}\right) \otimes V\left(\omega_{F}\right)$, hence the non-degeneracy of the multiplication of $M$ implies that $V_{D} \subset V_{E} V_{F}$ and it follows $V_{D^{\prime}+E_{0}^{\prime}+F_{0}^{\prime}} \subset V_{E^{\prime}+E_{0}^{\prime}} V_{F^{\prime}+F_{0}^{\prime}}$. On the other hand, by Corollary 1.7, $V_{D^{\prime}+E_{0}^{\prime}+F_{0}^{\prime}} \subset$ $V_{E^{\prime}+E_{0}^{\prime}} V_{F^{\prime}+F_{0}^{\prime}}=V_{E^{\prime}} V_{F^{\prime}} V_{E_{0}^{\prime}+F_{0}^{\prime}}$ if and only if $V_{D^{\prime}} \subset V_{E^{\prime}} V_{F^{\prime}}$.

Therefore, we have deduced that the multiplication of $M^{\prime}$ is non-degenerate, but this is a contradiction by the previous example together with Proposition 1.6.

Remark 2. More generally, if $M$ is a wonderful variety whose multiplication is nondegenerate and if $M^{\prime} \subset M$ is a localization of $M$, notice that the multiplication of $M^{\prime}$ is non-degenerate as well. If indeed $\rho: \operatorname{Pic}(M) \longrightarrow \operatorname{Pic}\left(M^{\prime}\right)$ is the restriction of line bundles and if $D, E \in \mathbb{N} \Delta$, then $\rho\left(V_{D} V_{E}\right)=V_{\rho(D)} V_{\rho(E)}$, and if $V_{F} \subset V_{D} V_{E}$, then $\rho\left(V_{F}\right) \neq 0$ if and only if $\operatorname{supp}_{\Sigma}(D+E-F) \subset \Sigma^{\prime}$ (where $\Sigma^{\prime} \subset \Sigma$ are the spherical roots of $M^{\prime}$ ). On the other hand, given $E^{\prime}, F^{\prime} \in \mathbb{N} \Delta^{\prime}$, by making use of Corollary 1.7, one can reduce the description of the product $V_{E^{\prime}} V_{F^{\prime}}$ to the one corresponding to a pair $E^{\prime \prime}, F^{\prime \prime} \in \rho\left(\mathbb{N} \Delta\right.$ ) (where $\Delta^{\prime}$ is the set of colors of $M^{\prime}$ ).

As well, if $M \longrightarrow M^{\prime}$ is a quotient of $M$ by a distinguished set of colors and if $H \subset H^{\prime}$ are the corresponding spherical subgroups, by Corollary 1.4 it follows that the multiplication of $M^{\prime}$ is non-degenerate if that of $M$ is non-degenerate. Indeed, if we identify the set of colors $\Delta^{\prime}$ of $M^{\prime}$ with a subset of $\Delta$, then $V_{E} V_{F} \subset \mathbb{C}[G]^{\left(H^{\prime}\right)} \subset$ $\mathbb{C}[G]^{(H)}$ for all $E, F \in \mathbb{N} \Delta^{\prime}$.

By using Proposition 1.6, similar statements are easily proved for parabolic inductions and localizations at a parabolic subgroup (see e.g. [8, 1.1.4] for the definition of the latter). Therefore the non-degeneracy property of the multiplication of a wonderful variety is stable under the standard operations of localization, quotient by a distinguished subset of colors, parabolic induction, localization at a parabolic subgroup.

Given a spherical subgroup $H \subset G$, define its spherical closure $\bar{H}$ as the kernel of the action of the normalizer $N_{G}(H)$ on the set of colors of $G / H$. If $H$ is equal to its spherical closure, then we say that $H$ is spherically closed. By a theorem of F. Knop [29, Corollary 7.6], if $H$ is spherically closed, then $G / H$ admits a wonderful compactification. If $G$ is not simply laced, then not spherically closed spherical subgroups $H$ such that $G / H$ admits a wonderful compactification exists. The projection $G / H \longrightarrow G / \bar{H}$ canonically identifies the colors of $G / \bar{H}$ with those of $G / H$. 
Generally speaking, if $M$ is the wonderful compactification of $G / H$ where $H$ is not spherically closed, then the multiplication may be degenerate. Indeed, it is easy to show that $\mathbb{C}[G]^{(H)}=\mathbb{C}[G]^{(\bar{H})}$, therefore, if $\bar{\Sigma}$ is the set of spherical roots of $G / \bar{H}$ and if $D \in \mathbb{N} \Delta$ is such that $V_{D} \subset V_{E} V_{F}$, then we must have $D \leqslant_{\bar{\Sigma}} E+F$. In this way we may construct examples of non-spherically closed spherical subgroups $H$ of $G$ (possessing no simple spherical roots) whose associated multiplication is degenerate.

Example 2. Consider the non-adjoint symmetric wonderful variety $M$ for $\operatorname{Sp}(8)$ with spherical roots $\sigma_{1}=\alpha_{1}+2 \alpha_{2}+\alpha_{3}, \sigma_{2}=\alpha_{3}+\alpha_{4}$. Then $M$ possesses two colors $D_{2}$ and $D_{4}$, where $\omega_{D_{2}}=\omega_{2}$ and $\omega_{D_{4}}=\omega_{4}$. Then we have $D_{2}<_{\Sigma} 2 D_{2}$ and $V\left(\omega_{2}\right) \subset V\left(\omega_{2}\right)^{\otimes 2}$, on the other hand $\bar{\Sigma}=\left\{\sigma_{1}, 2 \sigma_{2}\right\}$ and $2 D_{2}-D_{2}=\sigma_{1}+\sigma_{2} \notin \mathbb{N} \bar{\Sigma}$, therefore it cannot be $V_{D_{2}} \subset V_{D_{2}}^{2}$.

Proposition 9.2. Let $\mathscr{M} \subset \mathbb{N} \Sigma$ be the semigroup generated by the set of differences $\left\{E+F-D: V_{D} \subset V_{E} V_{F}\right\}$. Then $\mathscr{M}=\mathbb{N} \bar{\Sigma}$.

Proof. As already noticed we have $\mathscr{M} \subset \mathbb{N} \bar{\Sigma}$. Here we show that indeed $\mathscr{M} \supset$ $\bar{\Sigma}$. Since $\mathbb{C}[G]^{(H)}=\mathbb{C}[G]^{(\bar{H})}$ we may assume that $H$ is spherically closed. Let $\sigma \in \Sigma$, proceeding by localization and parabolic induction as in Proposition 9.1 we may assume that $M$ is a cuspidal (i.e., parabolic induction of no other wonderful variety) rank one wonderful variety with spherical root $\sigma$ (see [44, Table 1] for a complete list of such varieties). With three exceptions (cases 9B, 9C, 15 in [44]) such wonderful varieties are all flag varieties for their automorphism group, so that they are projectively normal. Being $H$ spherically closed, notice that apart from the cases $9 \mathrm{~B}, 7 \mathrm{C}, 9 \mathrm{C}, 12,15$ in 44 we have that $\sigma=D+E$ for some $D, E \in \Delta$; then $(0, D, E)$ is a low triple and the projective normality of $M$ implies that $V_{0} \subset V_{D} V_{E}$, hence $\sigma \in \mathscr{M}$. Moreover, since the case $7 \mathrm{C}$ is a quotient of the case $9 \mathrm{C}$, we are reduced to the cases $9 \mathrm{~B}, 7 \mathrm{C}, 12$ and 15, where the following inclusions may be checked directly (we index the colors as the corresponding fundamental weights):

9B) $G$ is of type $\mathrm{B}_{r}, \sigma=\alpha_{1}+\ldots+\alpha_{r}=D_{1}$ and we have $V_{D_{r}} \subset V_{D_{1}} V_{D_{r}}$.

7C) $G$ is of type $C_{r}, \sigma=\alpha_{1}+2 \alpha_{2}+\ldots+2 \alpha_{r-1}+\alpha_{r}=D_{2}$ and we have $V_{D_{2}} \subset V_{D_{2}}^{2}$.

12) $G$ is of type $\mathrm{F}_{4}, \sigma=\alpha_{1}+2 \alpha_{2}+3 \alpha_{3}+2 \alpha_{4}=D_{4}$ and we have $V_{D_{4}} \subset V_{D_{4}}^{2}$.

15) $G$ is of type $\mathrm{G}_{2}, \sigma=\alpha_{1}+\alpha_{2}=D_{2}-D_{1}$ and we have $V_{2 D_{1}} \subset V_{D_{1}} V_{D_{2}}$.

Suppose now that $M$ is a strict wonderful variety and suppose that $E, F \in \mathbb{N} \Delta$ are such that $E+F$ is not faithful. The following example shows that the product $V_{E} V_{F}$ may be degenerate, essentially reducing to a not spherically closed case.

Example 3. Let $M$ be the model wonderful variety of type $C_{4}$. Then $D_{2}<_{\Sigma} 2 D_{2}$ and $V\left(\omega_{2}\right) \subset V\left(\omega_{2}\right)^{\otimes 2}$ (more precisely, $V\left(\omega_{2}\right)$ is also contained in the second symmetric power of $\left.V\left(\omega_{2}\right)\right)$. Notice that $2 D_{2}$ is not faithful: the maximal distinguished subset of $\Delta$ which does not intersect the support of $2 D_{2}$ is $\Delta^{*}=\left\{D_{1}, D_{3}\right\}$, and the quotient $M / \Delta^{*}$ is the symmetric wonderful variety $M^{\prime}$ considered in Example 2 , whose colors we still be denoted by $D_{2}$ and $D_{4}$. By Corollary 1.4 we may identify $\Gamma\left(M, \mathcal{L}_{D_{2}}\right)=\Gamma\left(M^{\prime}, \mathcal{L}_{D_{2}}\right)$ and $\Gamma\left(M, \mathcal{L}_{2 D_{2}}\right)=\Gamma\left(M^{\prime}, \mathcal{L}_{2 D_{2}}\right)$, so that we may regard the product $V_{D_{2}}^{2}$ inside $\Gamma\left(M^{\prime}, \mathcal{L}_{2 D_{2}}\right)$ and by Example 2 it follows that $V_{D_{2}} \not \subset V_{D_{2}}^{2}$. 
Question. Let $M$ be a strict wonderful variety and let $E, F \in \mathbb{N} \Delta$ be such that $E+F$ is a faithful divisor. Is the product $V_{E} V_{F}$ non-degenerate?

Suppose that $G$ is simply laced: then the class of the strict wonderful varieties is stable with respect to the operation of quotient by a distinguished set of colors, so phenomena as that in Example 3 cannot happen. Therefore, if the answer to the previous question were affirmative, proceeding by induction on the rank of $M$, it would follow that the multiplication is non-degenerate whenever $M$ is a strict wonderful variety for a simply laced group.

\section{ACKNOWLEDGMENTS}

We would like to thank Domingo Luna, who suggested some years ago that we study the normality of spherical nilpotent orbit closures via the projective normality of wonderful varieties.

The paper was partially written during a stay of the second author at FriedrichAlexander-Universität Erlangen-Nürnberg between March and October 2012, partially supported by a DAAD fellowship. He is grateful to the Emmy Noether Zentrum and especially to Friedrich Knop for their warm hospitality. Also, many thanks are due to Friedrich Knop for fruitful discussions.

\section{REFERENCES}

[1] J. Adams, J.-S. Huang, and D. A. Vogan Jr., Functions on the model orbit in $E_{8}$, Represent. Theory 2 (1998), 224-263 (electronic), DOI 10.1090/S1088-4165-98-00048-X. MR.1628031 (99g:20077)

[2] D. Ahiezer, Equivariant completions of homogeneous algebraic varieties by homogeneous divisors, Ann. Global Anal. Geom. 1 (1983), no. 1, 49-78, DOI 10.1007/BF02329739. MR739893 $(85 \mathrm{j}: 32052)$

[3] D. Barbasch and D. A. Vogan Jr., Unipotent representations of complex semisimple groups, Ann. of Math. (2) 121 (1985), no. 1, 41-110, DOI 10.2307/1971193. MR782556 (86i:22031)

[4] I. N. Bernštel̆n, I. M. Gel'fand, and S. I. Gel'fand, Models of representations of compact Lie groups (Russian), Funkcional. Anal. i Priložen. 9 (1975), no. 4, 61-62. MR0414792 (54 \#2884)

[5] P. Bravi, Primitive spherical systems, Trans. Amer. Math. Soc. 365 (2013), no. 1, 361-407, DOI 10.1090/S0002-9947-2012-05621-2. MR2984062

[6] P. Bravi and S. Cupit-Foutou, Classification of strict wonderful varieties (English, with English and French summaries), Ann. Inst. Fourier (Grenoble) 60 (2010), no. 2, 641-681. MR2667789 (2011m:14085)

[7] P. Bravi, J. Gandini, A. Maffei, and A. Ruzzi, Normality and non-normality of group compactifications in simple projective spaces (English, with English and French summaries), Ann. Inst. Fourier (Grenoble) 61 (2011), no. 6, 2435-2461 (2012), DOI 10.5802/aif.2679. MR2976317

[8] P. Bravi and D. Luna, An introduction to wonderful varieties with many examples of type $\mathrm{F}_{4}$, J. Algebra 329 (2011), 4-51, DOI 10.1016/j.jalgebra.2010.01.025. MR2769314 (2012f:14102)

[9] P. Bravi and G. Pezzini, Wonderful varieties of type D, Represent. Theory 9 (2005), 578-637 (electronic), DOI 10.1090/S1088-4165-05-00260-8. MR2183057 (2006g:14078)

[10] M. Brion, Groupe de Picard et nombres caractéristiques des variétés sphériques (French), Duke Math. J. 58 (1989), no. 2, 397-424, DOI 10.1215/S0012-7094-89-05818-3. MR1016427 (90i:14048)

[11] M. Brion, The total coordinate ring of a wonderful variety, J. Algebra 313 (2007), no. 1, 61-99, DOI 10.1016/j.jalgebra.2006.12.022. MR2326138(2008d:14067)

[12] A. Broer, Decomposition varieties in semisimple Lie algebras, Canad. J. Math. 50 (1998), no. 5, 929-971, DOI 10.4153/CJM-1998-048-6. MR.1650954 (99k:14077)

[13] R. Chirivì, C. de Concini, and A. Maffei, On normality of cones over symmetric varieties, Tohoku Math. J. (2) 58 (2006), no. 4, 599-616. MR2297202 (2008g:14087) 
[14] R. Chirivì, P. Littelmann, and A. Maffei, Equations defining symmetric varieties and affine Grassmannians, Int. Math. Res. Not. IMRN 2 (2009), 291-347, DOI 10.1093/imrn/rnn132. MR2482117(2010b:14104)

[15] R. Chirivì and A. Maffei, Projective normality of complete symmetric varieties, Duke Math. J. 122 (2004), no. 1, 93-123, DOI 10.1215/S0012-7094-04-12213-4. MR2046808(2005b:14085)

[16] R. Chirivì and A. Maffei, Plücker relations and spherical varieties: application to model varieties, Transform. Groups 19 (2014), no. 4, 979-997, DOI 10.1007/s00031-014-9285-6. MR.3278858

[17] D. H. Collingwood and W. M. McGovern, Nilpotent orbits in semisimple Lie algebras, Van Nostrand Reinhold Mathematics Series, Van Nostrand Reinhold Co., New York, 1993. MR.1251060 (94j:17001)

[18] M. Costantini, On the coordinate ring of spherical conjugacy classes, Math. Z. 264 (2010), no. 2, 327-359, DOI 10.1007/s00209-008-0468-5. MR2574980 (2010k:20071)

[19] C. De Concini, Normality and non normality of certain semigroups and orbit closures, Algebraic transformation groups and algebraic varieties, Encyclopaedia Math. Sci., vol. 132, Springer, Berlin, 2004, pp. 15-35, DOI 10.1007/978-3-662-05652-3_3. MR2090668 (2005g:20068)

[20] C. De Concini and C. Procesi, Complete symmetric varieties, Invariant theory (Montecatini, 1982), Lecture Notes in Math., vol. 996, Springer, Berlin, 1983, pp. 1-44, DOI 10.1007/BFb0063234. MR718125 (85e:14070)

[21] W. A. de Graaf, Constructing representations of split semisimple Lie algebras, J. Pure Appl. Algebra 164 (2001), no. 1-2, 87-107, DOI 10.1016/S0022-4049(00)00150-X. Effective methods in algebraic geometry (Bath, 2000). MR1854331 (2002h:17007)

[22] J. Gandini, Spherical orbit closures in simple projective spaces and their normalizations, Transform. Groups 16 (2011), no. 1, 109-136, DOI 10.1007/s00031-011-9120-2. MR2785497 (2012f:14091)

[23] The GAP Group, Aachen, St Andrews, GAP-Groups, Algorithms, and Programming - a System for Computational Discrete Algebra, Version 4.5.4, 2012. http://www-gap.dcs.stand.ac.uk/ gap

[24] I. M. Gel'fand and A. V. Zelevinskǐ̌, Models of representations of classical groups and their hidden symmetries (Russian), Funktsional. Anal. i Prilozhen. 18 (1984), no. 3, 14-31. MR757246(86i:22024)

[25] I. M. Gel'fand and A. V. Zelevinsky, Representation models for classical groups and their higher symmetries, Astérisque Numero Hors Serie (1985), 117-128. The mathematical heritage of Élie Cartan (Lyon, 1984). MR837197 (88b:22022)

[26] W. Hesselink, The normality of closures of orbits in a Lie algebra, Comment. Math. Helv. 54 (1979), no. 1, 105-110, DOI 10.1007/BF02566258. MR.522033 (80g:14018)

[27] S. S. Kannan, Projective normality of the wonderful compactification of semisimple adjoint groups, Math. Z. 239 (2002), no. 4, 673-682, DOI 10.1007/s002090100319. MR.1902056 (2003f:20076)

[28] M. Kapovich and J. J. Millson, Structure of the tensor product semigroup, Asian J. Math. 10 (2006), no. 3, 493-539, DOI 10.4310/AJM.2006.v10.n3.a2. MR2253157 (2007h:22009)

[29] F. Knop, Automorphisms, root systems, and compactifications of homogeneous varieties, J. Amer. Math. Soc. 9 (1996), no. 1, 153-174, DOI 10.1090/S0894-0347-96-00179-8. MR.1311823 (96c:14037)

[30] F. Knop, H. Kraft, and T. Vust, The Picard group of a G-variety, Algebraische Transformationsgruppen und Invariantentheorie, DMV Sem., vol. 13, Birkhäuser, Basel, 1989, pp. 77-87. MR.1044586

[31] A. Knutson and T. Tao, The honeycomb model of $\mathrm{GL}_{n}(\mathbf{C})$ tensor products. I. Proof of the saturation conjecture, J. Amer. Math. Soc. 12 (1999), no. 4, 1055-1090, DOI 10.1090/S08940347-99-00299-4. MR:1671451 (2000c:20066)

[32] B. Kostant and S. Rallis, Orbits and representations associated with symmetric spaces, Amer. J. Math. 93 (1971), 753-809. MR0311837 (47 \#399)

[33] S. Kumar, Tensor product decomposition, Proceedings of the International Congress of Mathematicians. Volume III, Hindustan Book Agency, New Delhi, 2010, pp. 1226-1261. MR2827839

[34] D. Luna, Toute variété magnifique est sphérique (French, with English summary), Transform. Groups 1 (1996), no. 3, 249-258, DOI 10.1007/BF02549208. MR1417712(97h:14066) 
[35] D. Luna, Variétés sphériques de type A (French), Publ. Math. Inst. Hautes Études Sci. 94 (2001), 161-226, DOI 10.1007/s10240-001-8194-0. MR1896179(2003f:14056)

[36] D. Luna, La variété magnifique modèle (French, with English and French summaries), J. Algebra 313 (2007), no. 1, 292-319, DOI 10.1016/j.jalgebra.2006.10.042. MR2326148 (2008e:14070)

[37] A. Maffei, Orbits in degenerate compactifications of symmetric varieties, Transform. Groups 14 (2009), no. 1, 183-194, DOI 10.1007/s00031-008-9040-y. MR2480858(2010f:14055)

[38] D. I. Panyushev, Some amazing properties of spherical nilpotent orbits, Math. Z. 245 (2003), no. 3, 557-580, DOI 10.1007/s00209-003-0555-6. MR2021571 (2004j:14051)

[39] G. Pezzini, Simple immersions of wonderful varieties, Math. Z. 255 (2007), no. 4, 793-812, DOI 10.1007/s00209-006-0050-y. MR2274535(2007j:14069)

[40] R. W. Richardson, On orbits of algebraic groups and Lie groups, Bull. Austral. Math. Soc. 25 (1982), no. 1, 1-28, DOI 10.1017/S0004972700005013. MR651417 (83i:14041)

[41] J. R. Stembridge, The partial order of dominant weights, Adv. Math. 136 (1998), no. 2, 340-364, DOI 10.1006/aima.1998.1736. MR1626860(2000a:06038)

[42] D. A. Timashev, Homogeneous spaces and equivariant embeddings, Encyclopaedia of Mathematical Sciences, vol. 138, Springer, Heidelberg, 2011. Invariant Theory and Algebraic Transformation Groups, 8. MR2797018 (2012e:14100)

[43] D. A. Vogan Jr., The method of coadjoint orbits for real reductive groups, Representation theory of Lie groups (Park City, UT, 1998), IAS/Park City Math. Ser., vol. 8, Amer. Math. Soc., Providence, RI, 2000, pp. 179-238. MR1737729 (2001k:22027)

[44] B. Wasserman, Wonderful varieties of rank two, Transform. Groups 1 (1996), no. 4, 375-403, DOI 10.1007/BF02549213. MR1424449 (97k:14051)

Dipartimento di Matematica, Sapienza Università di Roma, Piazzale Aldo Moro 5 , 00185 RoMA, ITALY

E-mail address: bravi@mat.uniroma1.it

Scuola Normale Superiore, Piazza dei Cavalieri 7, 56126 Pisa, Italy

E-mail address: jacopo.gandini@sns.it

Dipartimento di Matematica, Università di Pisa, Largo Bruno Pontecorvo 5, 56127 Pisa, ITALy

E-mail address: maffei@dm.unipi.it 